\title{
O índice de Poincaré-Hopf e generalizações no caso singular
}


SERVIÇO DE PÓS-GRADUAÇÃO DO ICMC-USP

Data de Depósito: 10/03/2011

Assinatura:

\section{O índice de Poincaré-Hopf e generalizações no caso singular}

Thaís Maria Dalbelo

Orientador: Prof. Dr. Nivaldo de Góes Grulha Júnior

Dissertação apresentada ao Instituto de Ciências Matemáticas e de Computação - ICMC-USP, como parte dos requisitos para obtenção do título de Mestre em Ciências - Matemática. VERSÃO REVISADA.

USP - São Carlos

Março/2011 
Ficha catalográfica elaborada pela Biblioteca Prof. Achille Bassi e Seção Técnica de Informática, ICMC/USP, com os dados fornecidos pelo(a) autor(a)

\begin{tabular}{|c|c|}
\hline \multirow[t]{3}{*}{ D137í } & $\begin{array}{l}\text { Dalbelo, Thaís Maria } \\
\text { O índice de Poincaré-Hopf e generalizações no caso } \\
\text { singular / Thaís Maria Dalbelo; orientador Nivaldo de } \\
\text { Góes Grulha Júnior -- São Carlos, } 2011 \text {. } \\
\quad 143 \mathrm{p} \text {. }\end{array}$ \\
\hline & $\begin{array}{l}\text { Dissertação (Mestrado - Programa de Pós-Graduação en } \\
\text { Matemática)-- Instituto de Ciências Matemáticas e } \\
\text { de Computação, Universidade de São Paulo, } 2011 .\end{array}$ \\
\hline & $\begin{array}{l}\text { 1. Variedades singulares. 2. Índice de Poincaré- } \\
\text { Hopf. 3. Índice de Schwartz. 4. Índice GSV. } 5 . \\
\text { Hipersuperfície com singularidade isolada. I. Grulha } \\
\text { Júnior, Nivaldo de Góes, orient. II. Título. }\end{array}$ \\
\hline
\end{tabular}


Aos meus avós Joaquim, Maria, Osório e Italina (In memoriam).

$E$ aos meus amados pais Arcangelo e Iria. 


\section{O que, afinal, aprendemos?}

Chega um tempo em que, afinal, você aprende que amar não significa apoiar-se.

E passa a aceitar suas derrotas com a cabeça erguida e os olhos adiante, com a graça de um adulto, e não com a tristeza de uma criança.

Depois de um tempo, você aprende que não importa quanto você se importe, algumas pessoas simplesmente não se importam.

E aceita que, não importa quão boa seja uma pessoa, ela vai feri-lo de vez em quando e você precisa perdoa-la por isso.

Aprende que verdadeiras amizades continuam a crescer mesmo quando separadas por longas distâncias.

E que o que importa não é o que você tem na vida, mas quem você tem na vida.

Aprende que não temos de mudar de amigos se compreendermos que os amigos mudam; percebe que seu melhor amigo e você podem fazer qualquer coisa, ou nada, e ter bons momentos juntos.

Começa a aprender que não deve comparar-se aos outros, mas ao melhor que você pode ser.

Descobre que demora muito tempo para alguém se tornar a pessoa que quer ser e que o tempo é curto.

Aprende que ser flexível não significa ser fraco ou não ter personalidade, pois não importa quão delicada e frágil seja uma situação, sempre existem dois lados.

Aprende que paciência requer muita prática.

Descobre que algumas vezes a pessoa que você espera que o chute quando cai é uma das poucas que o ajudarão a se levantar.

Aprende que há mais dos seus pais em você do que supunha.

Aprende que, quando você está com raiva, pode até ter o direito de estar com raiva, mas não de ser cruel.

Aprende que nem sempre é suficiente ser perdoado por alguém. Algumas vezes, você tem de aprender a perdoar a si mesmo.

Aprende que, com a mesma serenidade com que julga, você será em algum momento condenado. 
Aprende que, não importa em quantos pedaços seu coração se tenha partido, o mundo não vai parar para que você o conserte.

Aprende que o tempo não é algo que possa voltar atrás.

Pois você aprende que realmente pode suportar, que realmente é forte e que pode ir ainda muito mais longe depois de ter pensado que não poderia mais.

Portanto, plante seu jardim e decore sua alma em vez de esperar que alguém lhe traga flores.

William Shakespeare 



\section{Agradecimentos}

Desde o ínicio da minha graduação desejei levar meus estudos adiante, e o que aos meus olhos parecia quase impossível foi aos poucos se tornando realidade! Mas nessa minha longa jornada de aprendizado contei com o enorme apoio de diversas pessoas que contribuiram de várias maneiras. Por isso, gostaria de agradecer...

À Deus, pela minha vida, pela minha família, por ter me dado força, coragem e fé e por ter me presenteado com as pessoas maravilhosas que cruzaram meu caminho.

Aos meus pais, por sempre estarem ao meu lado, mesmo que em pensamentos e orações. À eles que sempre me mostraram o verdadeiro significado do amor e da dignidade. À eles, que sempre priorizaram a educação dos filhos, e que muitas vezes deixaram de realizar suas vontades para que eu e meu irmão seguissemos em frente em nossos estudos. À eles, que eu tanto amo, deixo meu amor e minha eterna gratidão.

Ao meu irmão Luiz Fernando, que depois de nossas eternas brigas sempre foi um grande amigo.

Aos meus amigos Alex Carlucci Rezende, Vinícius Casteluber Laass e Flávio de Souza Pires, pelas dezenas de vezes que pedi a ajuda de vocês e pelas centenas de vezes em que vocês se dispuseram a me ajudar. Pelas alegrias somadas e problemas compartilhados. Por me fazerem sentir que eu era capaz, quando muitas vezes nem eu mesma acreditava, pelos puxões de orelha, pela confiança. Enfim, por tudo.

Aos amigos Andreza, Apoenã, Camila, Henry, Juliana, Matheus, Nelson, Northon, Rafael Borro, Rafael Figueiredo e Renato Fehlberg, pela ajuda nos estudos, pelos passeios, pelos deliciosos churrascos e almoços, por todos os momentos que tivemos juntos. 
Às minhas inesquecíveis companheiras em Presidente Prudente, Márcia, Gislaine, Vanderlea, Renata e Jéssica, foi muito bom ter convivido com vocês.

Ao meu orientador Nivaldo, pela enorme atenção, pela paciência em responder várias vezes a mesma pergunta, por nunca ter poupado esforços para sanar minhas dúvidas, muitas vezes sacrificando até seu tempo entre uma aula e um café para poder me ajudar. Pela correção minuciosa desta dissertação e por ser um grande amigo, me ajudando com conselhos e valiosas sugestões. Sua humildade e sua paixão pela matemática sempre serão exemplos para mim. Muito obrigada por tudo.

Ao professor Jean-Paul Brasselet, que mesmo com tantos compromissos importantes, encontrou tempo para tirar minhas dúvidas sempre que esteve no Brasil, me ajudando com muito dedicação e paciência.

Ao professor Marcelo Messias, cuja contribuição em toda minha graduação foi, sem dúvida alguma, essencial.

Ao professor Rodrigo Martins, por toda a atenção dedicada a mim enquanto estive em Maringá. Você me ajudou a dar um grande passo.

À todos os professores e funcinários das instituicões de ensino que fui aluna, muito obrigada.

À Fapesp e à Capes pelo apoio financeiro para realização deste trabalho. 


\section{Resumo}

Neste trabalho, estudamos o índice de Poincaré-Hopf, definido para singularidades isoladas de campos de vetores sobre variedades diferenciáveis. Além disso, investigamos algumas definições de índices de campos de vetores definidos em variedades singulares, como o índice de Schwartz e o índice GSV. Estudaremos estes invariantes no caso específico em que $(V, 0)$ é um germe de uma interseção completa com singularidade isolada na origem. 



\section{Abstract}

In this work, we study the Poincaré-Hopf index, defined for isolated singularities of vector fields on manifolds. Moreover, we investigate some definitions of indices of vector fields defined on singular varieties, as the Schwartz index and the GSV index. We study these invariants in the case where $(V, 0)$ is a germ of a complete intersection with an isolated singularity at the origin. 



\section{Sumário}

$\begin{array}{ll}\text { Introdução } & 13\end{array}$

\begin{tabular}{lll}
\hline & Preliminares & 17
\end{tabular}

1.1 Variedades diferenciáveis . . . . . . . . . . . . . . . . . . . . . . . . 17

1.2 Homotopia . . . . . . . . . . . . . . . . . . . . . . . . . . 27

1.3 Homologia singular . . . . . . . . . . . . . . . . . . . . . . 29

1.4 Teoria de singularidades $\ldots \ldots \ldots \ldots$. . . . . . . . . . . . 34

1.5 Variedades algébricas e espaços analíticos complexos . . . . . . . . . . . . . 36

$2 \quad$ O índice de Poincaré-Hopf para campos de vetores em $\mathbb{R}^{2} \quad 41$

2.1 O índice de Poincaré-Hopf . . . . . . . . . . . . . . . . . . . . 45

3 Grau de uma aplicação $\quad 51$

3.1 Grau de uma aplicação . . . . . . . . . . . . . . . . . . . . . . . . 53

3.2 Grau local de uma aplicação . . . . . . . . . . . . . . . . . . . . . . . 73

4 Índice de Poincaré-Hopf

4.1 Campos de vetores . . . . . . . . . . . . . . . . . . . . . . . 84

4.2 O índice de Poincaré-Hopf $\ldots \ldots \ldots$. . . . . . . . . . . . . 91

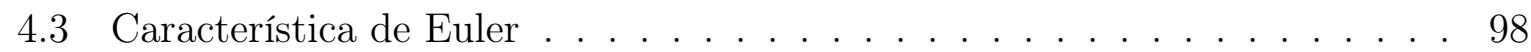

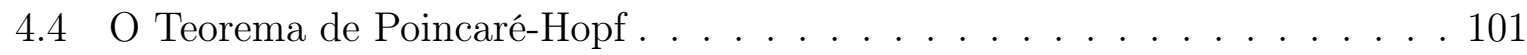


5 Índices de campos de vetores em variedades singulares 115

5.1 O índice de Schwartz . . . . . . . . . . . . . . . . . . . . . . . . 119

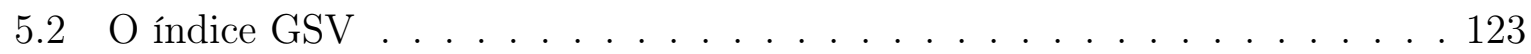

$\begin{array}{lll}6 \text { Conclusão } & 139\end{array}$ 


\section{Introdução}

Seja $v$ um campo vetorial em $\mathbb{R}^{n}$, dizemos que $p \in \mathbb{R}^{n}$ é um ponto singular ou uma singularidade de $v$ quando $v(p)=\overrightarrow{0}$. O estudo de campos de vetores (e fluxos) na vizinhança de uma singularidade, ou ponto estacionário, é um tema amplamente estudado devido a sua importância em vários ramos da matemática e também por suas inúmeras aplicações em outras áreas.

$\mathrm{Na}$ biologia, podemos citar o campo de vetores conhecido como presa-predador, que tem sido estudado no controle biológico de pragas de canaviais, tal campo descreve um modelo de interação entre duas espécies de animais, uma das quais se alimenta da outra. $\mathrm{Na}$ física, são inúmeros os problemas envolvendo campo de vetores, podemos citar por exemplo a modelagem do movimento de uma partícula em um dado fluido.

Já na meteorologia, um importante exemplo é o modelo matemático proposto pelo meteorologista americano E. N. Lorenz em 1963 para descrever o movimento convectivo de fluidos na atmosfera terrestre. O modelo proposto é um campo de vetores, que ficou conhecido na literatura como sistema de Lorenz. Alguns estudos feitos por meteorologistas mostraram que o comportamento desse campo pode sofrer mudanças drásticas nas proximidades de suas singularidades, o que nos ajuda a entender porque é tão difícil fazer previsões precisas do tempo.

Informações sobre o comportamento de um campo vetorial perto de um ponto singular $p$, podem ser obtidas através de alguns invariantes definidos para este ponto.

O invariante mais básico de um campo de vetores em uma singularidade isolada é o seu índice de Poincaré-Hopf, que além de ser um conceito extremamente importante, é 
considerado como um verdadeiro traço de união entre ramos distintos da matemática, como a Topologia Algébrica e a Topologia Diferencial.

Tal conexão só é possível por causa de um importante resultado, que foi demonstrado em dimensão 2 por H. Poincaré em 1885, e generalizado para dimensões maiores por H. Hopf em 1927, esse resultado é chamado de Teorema de Poincaré-Hopf.

Esse importante teorema diz que a característica de Euler de uma variedade diferenciável compacta $M$ é igual a soma dos índices de Poincaré-Hopf das singularidades isoladas de um campo de vetores contínuo $v$ definido em $M$, independente da escolha do campo $v$.

É cada vez mais usual encontrarmos problemas e situações em matemática e outras ciências, em que precisamos considerar campos de vetores definidos em objetos singulares. A pergunta natural então é a seguinte: Qual seria uma boa noção de índice de um campo de vetores sobre uma variedade singular?

Índices de campos de vetores em variedades singulares foram considerados primeiramente por M.-H. Schwartz, por volta de 1965, em seu estudo do Teorema de PoincaréHopf e de Classes de Chern para variedades singulares. O importante índice que M.-H. Schwartz definiu, e que ficou conhecido como índice de Schwartz possui uma importante propriedade que nos permite relacioná-lo com o índice de Poincaré-Hopf.

Mais especificamente, usando a sua definição de índice, ela demonstrou que o Teorema de Poincaré-Hopf continua válido em uma variedade singular (Teorema 5.1.4).

Uma das propriedades básicas do índice de Poincaré-Hopf é estabilidade sobre perturbações. Em outras palavras, se um campo de vetores $v$ definido em uma variedade diferenciável $M$ tem uma singularidade isolada e se o perturbamos levemente, então a singularidade $p$ do campo de vetores inicial $v$ poderá se dividir em vários pontos singulares, com a propriedade que a soma dos índices do campo de vetores perturbado em seus pontos singulares é igual ao índice do campo de vetores original $v$ em sua singularidade.

Um índice para campos de vetores sobre variedades singulares que tem essa propriedade é o índice GSV, introduzido nos anos 90 por X. Gómez-Mont, J. Seade e A. Verjovsky em [8] para germes de hipersuperfícies e extendido em [24] (ver também [25]) para interseções completas. 
A motivação dos autores para a definição deste índice foi uma questão a cerca de separatrizes de campos vetoriais levantada por C. Camacho, que buscavam obter, a partir de um campo de vetores holomorfo sem separatriz em uma superfície singular $S$ de $\mathbb{C}^{3}$, uma extenção de tal campo, para o espaço ambiente, de forma que esta extensão fosse tangente às fibras de Milnor, e estudar suas singularidades.

O principal objetivo deste trabalho é estudar estas noções de índices de campos de vetores definidos em variedades diferenciáveis e em variedades singulares.

No Capítulo 1, apresentamos algumas definições básicas que utilizaremos ao longo do texto. Procuramos nos limitar ao que for realmente necessário para a compreensão dos capítulos seguintes.

No Capítulo 2, estudaremos o índice de Poincaré-Hopf de uma singularidade isolada $p$ de um campo de vetores contínuo $v$ definido em $\mathbb{R}^{2}$. Primeiramente, daremos a definição de campo de vetores e apresentaremos alguns exemplos. Em seguida, definiremos o índice de Poincaré-Hopf de uma singularidade isolada $p$ de um campo de vetores $v$ em $\mathbb{R}^{2}$ e apresentaremos uma importante interpretação geométrica deste invariante. Vale ressaltar que este capítulo, nos servirá como uma motivação para a generalização de tal conceito.

Em seguida, estudaremos o grau de uma aplicação diferenciável. Com base em [16], definiremos o conceito de grau de uma aplicação própria, e em seguida apresentaremos algumas propriedades e exemplos de tal conceito. Na Seção 3.2, definiremos o grau local de uma aplicação.

No Capítulo 4, primeiramente estudaremos o conceito de campos de vetores definidos em variedades diferenciáveis. Na Seção 4.2 definiremos o índice de Poincaré-Hopf de uma singularidade isolada $p$ de um campo de vetores contínuo $v$ definido em uma variedade diferenciável $M$. Em seguida, fazeremos um estudo da característica de Euler de uma variedade diferenciável compacta $M$. Posteriormente, baseados em [16], provaremos o Teorema de Poincaré-Hopf para uma variedade diferenciável compacta, orientada e sem bordo $M$, e apresentaremos uma versão deste resultado no caso em que a variedade $M$ possui bordo.

O caso singular é estudado no Capítulo 5. Na seção 5.1, definiremos o índice de Schwartz e faremos uma demonstração do resultado que relaciona o índice de Schwartz 
com o índice de Poincaré-Hopf. Em seguida, apresentaremos o índice GSV, definido originalmente por X. Gómez-Mont, J. Seade e A. Verjovsky em [8, 23] e provaremos que o índice GSV de uma singularidade isolada de um campo de vetores tangente $v$ definido em uma variedade singular é igual a soma dos índices de Poincaré-Hopf das singularidades que surgem quando estendemos o campo $v$ para um campo de vetores $\tilde{v}$ tangente a fibra de Milnor $F$. Estudaremos o caso em que a variedade considerada é uma interseção completa com singularidade isolada (ICSI), além disso, com base em [3] calcularemos alguns exemplos deste índice e mostraremos que este índice nos permite recuperar o Teorema de Poincaré-Hopf no caso singular. 


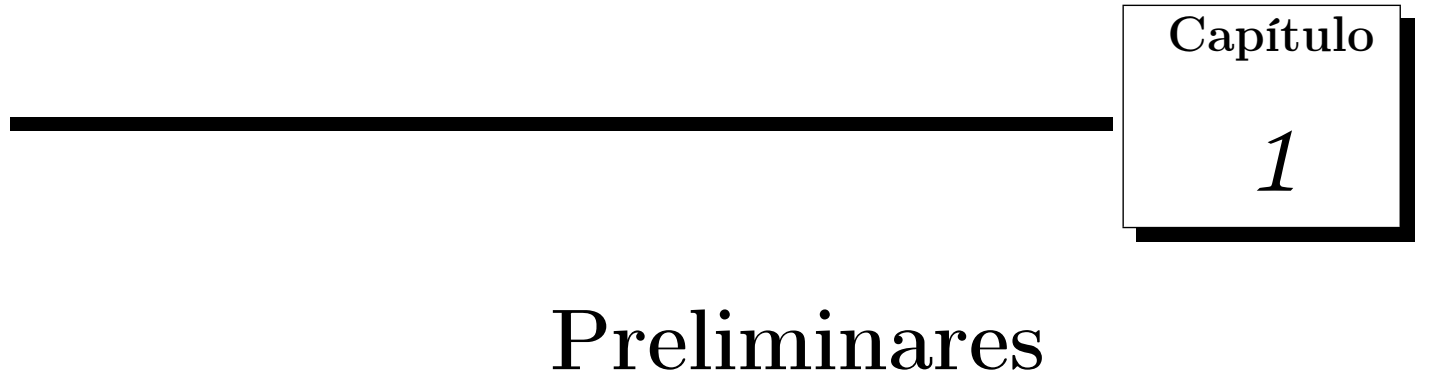

Pretendemos neste capítulo dar uma breve introdução aos principais conceitos usados no decorrer deste texto, tais como, variedades diferenciáveis, homotopia, homologia, variedades algébricas e variedades analíticas, procurando fornecer o mínimo necessário dessa teoria para uma boa compreensão dos resultados dos capítulos a seguir.

\subsection{Variedades diferenciáveis}

Neste trabalho, estudaremos alguns índices de campos vetoriais definidos sobre variedades diferenciáveis. Assim, nesta seção, daremos a definição de variedades diferenciáveis, estudaremos algumas de suas propriedades e veremos alguns conceitos importantes relacionados a variedades, tais como transversalidade e orientação.

Definição 1.1.1. Seja $M$ um espaço topológico. Um sistema de coordenadas locais ou uma carta em $M$ é um homeomorfismo $\varphi: U \rightarrow \varphi(U)$ de um subconjunto aberto $U \subset M$ sobre um aberto $\varphi(U) \subset \mathbb{R}^{m}$.

Definição 1.1.2. Um atlas $\mathcal{U} m$-dimensional de um espaço topológico $M$ é uma família de sistemas de coordenadas locais $\varphi: U \rightarrow \varphi(U) \subset \mathbb{R}^{m}$, tal que os domínios dos sistemas de coordenadas cubram $M$. 
Definição 1.1.3. Dados os sistemas de coordenadas locais $\varphi: U \rightarrow \mathbb{R}^{m}$ e $\psi: V \rightarrow \mathbb{R}^{m}$ no espaço topológico $M$, tais que $U \cap V \neq \emptyset$, então o homeoforfismo

$$
\phi_{\varphi \psi}=\psi \circ \varphi^{-1}: \varphi(U \cap V) \rightarrow \psi(U \cap V)
$$

é chamado mudança de coordenadas.

Observação 1.1.4. Seja a mudança de coordenadas $\phi_{\varphi \psi}=\psi \circ \varphi^{-1}$, então $\left(\psi \circ \varphi^{-1}\right)^{-1}=$ $\varphi \circ \psi^{-1}=\phi_{\psi \varphi}$ é também uma mudança de coordenadas.

Definição 1.1.5. Um atlas $\mathcal{U}$ em um espaço topológico $M$ é diferenciável de classe $C^{k}$, se todas as mudanças de coordenadas $\phi_{\varphi \psi}$ forem aplicações diferenciáveis de classe $C^{k}$.

Observação 1.1.6. Da Observação 1.1.4 e da Definição 1.1.5 segue que em um atlas diferenciável $\mathcal{U}$ todas as mudanças de coordenadas $\phi_{\varphi \psi}$ são difeomorfismos.

Definição 1.1.7. Uma variedade diferenciável $m$-dimensional, de classe $C^{k}$, é um par $(M, \mathcal{U})$, em que $M$ é um espaço topológico Hausdorff com base enumerável e $\mathcal{U}$ é um atlas $m$-dimensional de classe $C^{k}$.

Como vimos na Observação 1.1.6 em um atlas diferenciável $\mathcal{U}$ todas as mudanças de coordenadas $\phi_{\varphi \psi}$ são difeomorfismos. É exatamente esta propriedade que nos permite transportar todas as noções de Cálculo Diferenciál do $\mathbb{R}^{m}$ para as variedade diferenciáveis.

Seja $M$ uma variedade de classe $C^{k}$ e seja $p$ um ponto de $M$. Indicamos por $C_{p}$ o conjunto de todos os caminhos $\lambda: I \rightarrow M$, definidos num intervalo aberto $I$, contendo 0 , tais que $\lambda(0)=p$ e $\lambda$ é diferenciável em 0 . Diremos que dois caminhos $\lambda, \mu \in C_{p}$ são equivalentes, e escreveremos $\lambda \sim \mu$, quando existir um sistema de coordenadas locais $\varphi: U \rightarrow \mathbb{R}^{m}$ em $M$, com $p \in U$, tal que $(\varphi \circ \lambda)^{\prime}(0)=(\varphi \circ \mu)^{\prime}(0)$. Como as mudanças de coordenadas são difeomorfismo, a igualdade $(\varphi \circ \lambda)^{\prime}(0)=(\varphi \circ \mu)^{\prime}(0)$ será verdadeira para todo sistema de coordenada $\varphi: U \rightarrow \mathbb{R}^{m}$ em $M, p \in U$. Resulta daí que a relação acima é uma relação de equivalência.

O vetor velocidade $\lambda^{\prime}$ de um caminho $\lambda \in C_{p}$ é, por definição, a classe de equivalência de $\lambda$. Ou seja, $\lambda^{\prime}=\left\{\mu \in C_{p}: \mu \sim \lambda\right\}$. 
Definição 1.1.8. Definimos o espaço tangente à variedade $M$ no ponto $p$ como sendo o conjunto quociente $C_{p} / \sim$. Denotaremos o espaço tangente a variedade $M$ no ponto $p$ por $T_{p} M$.

Podemos mostrar que o espaço tangente a uma variedade diferenciável $M$ em um ponto $p \in M$ é um espaço vetorial de mesma dimensão que $M$.

Dada uma variedade diferenciável $m$-dimensional $M$, podemos obter uma nova variedade $2 m$-dimensional a partir de uma "união" dos espaços tangentes $T_{p} M$, em todos os pontos $p \in M$.

Definição 1.1.9. Seja $M$ uma variedade diferenciável de dimensão $m$. O conjunto

$$
T M=\bigcup_{p \in M}\{p\} \times T_{p} M
$$

de todos os espaços tangente $T_{p} M$, com $p \in M$, "colados" de uma maneira natural é chamado de fibrado tangente de $M$.

Proposição 1.1.10. Dada uma variedade diferenciável $m$-dimensional $M$, o fibrado tangente $T M$ tem naturalmente a estrutura de uma variedade $2 m$-dimensional.

Informalmente, o fibrado tangente de uma variedade é obtido por considerar-se todos os espaços tangentes, e reuní-los em um conjunto diferenciável e sem sobreposição, como ilustra a Figura 1.1 onde a variedade em questão é o círculo unitário em $\mathbb{R}^{2}$.
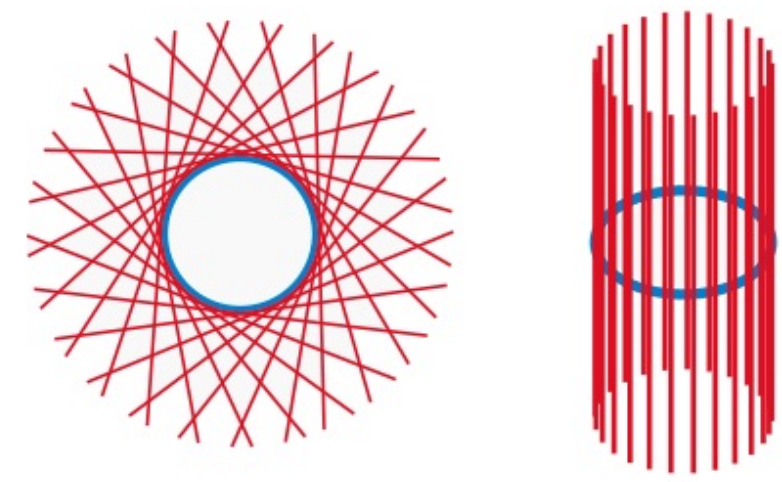

Figura 1.1: Representação do fibrado tangente de $\mathbb{S}^{1}$

Agora, que já vimos a definição de espaço vetorial tangente, podemos definir o conceito de diferenciabilidade de uma aplicação $f: M \rightarrow N$ entre variedades diferenciáveis, 
utilizando nosso conhecimento prévio a cerca das aplicações deferenciáveis definidas em um subconjunto aberto de $\mathbb{R}^{m}$ com valores em $\mathbb{R}^{n}$.

Definição 1.1.11. Sejam $M, N$ variedades diferenciáveis e uma aplicação $f: M \rightarrow N$, dizemos que $f$ é diferenciável em $p \in M$ se existem cartas $\varphi: U_{1} \rightarrow V_{1}$ para $M$ com $p \in U_{1}$ e $\psi: U_{2} \rightarrow V_{2}$ para $N \operatorname{com} f\left(U_{1}\right) \subset U_{2}$ tal que $\psi \circ f \circ \varphi^{-1}$ é diferenciável em $\varphi(p)$. Diremos simplesmente que $f$ é diferenciável se ela o for em todos os pontos de $M$. E chamaremos $f$ uma aplicação de classe $C^{k}$ quando $\psi \circ f \circ \varphi^{-1}$ for de classe $C^{k}$.

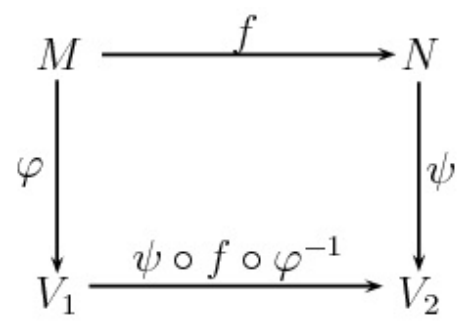

Podemos provar que a definição acima não depende das escolhas das parametrizações $\varphi$ e $\psi$, sendo então coerente.

Neste trabalho, as variedades diferenciáveis conexas serão de grande importância, vejamos então, um resultado relacionado a tais variedades.

Teorema 1.1.12. Seja $M$ uma variedade diferencíavel $n$-dimensional conexa. Dados dois pontos quaisquer $p, q \in M$, existe uma carta $\varphi: V \rightarrow \mathbb{R}^{n}$ de $M$, com $p, q \in V$, tal que, $\varphi(V)=\mathbb{R}^{n}$.

Nem sempre é fácil verificar se um espaço topológico $M$ é uma variedade diferenciável, porém, existe um importante resultado relacionado ao conceito de valor regular que nos ajuda a decidir se $M$ é uma variedade diferenciável.

Definição 1.1.13. Sejam $M$ uma variedade diferenciável $m$-dimensional, $N$ uma variedade diferenciável $n$-dimensional e $f: M \rightarrow N$ uma aplicação diferenciável. Dizemos que:

(1) $f$ é uma imersão em $p$ se a aplicação derivada $d f_{p}$ é injetiva (portanto $n \geq m$ ) em $p \in M$. Isto é, o posto da matriz jacobiana $[J f(p)]_{n \times m}$ é igual a $m$; 
(2) $f$ é uma submersão em $p$ se a aplicação derivada $d f_{p}$ é sobrejetora (portanto $m \geq n$ ) em $p \in M$. Isto é, o posto da matriz jacobiana $[J f(p)]_{n \times m}$ é igual a $n$.

Se em $p \in M$ a aplicação $f: M \rightarrow N$ for uma submersão, $p$ é dito ponto regular de $f$. Um ponto $q \in N$ é chamado valor regular de $f$ se sua imagem inversa $f^{-1}(q)$ só contém pontos regulares.

Se em $p \in M$ a aplicação $f: M \rightarrow N$ não é submersão e nem imersão, então $p$ é dito ponto singular de $f$ e $f(p)$ é o valor singular de $f$.

Teorema 1.1.14. Sejam $N$ uma variedade diferenciável $n$-dimensional, $P$ uma variedade diferenciável $p$-dimensional, $f: N \rightarrow P$ uma aplicação diferenciável e $p \in P$ um valor regular de $f$. Então, $f^{-1}(p)=M$ é uma subvariedade $(n-p)$-dimensional de $N$ e dado $q \in M$, temos $T_{q} M=\operatorname{Ker}\left(d f_{q}\right)$, onde $d f_{q}$ representa a aplicação derivada de $f$ no ponto $q$.

Dada uma aplicação $f: M \rightarrow N$ de classe $C^{1}$, existe um resultado importantíssimo acerca dos valores regulares da aplicação $f$. Este resultado é conhecido como Teorema de Sard e nos será indispensável no Capítulo 3.

Teorema 1.1.15. (Teorema de Sard) Seja $f: M \rightarrow N$ uma aplicação de classe $C^{1}$, o subconjunto formado pelos valores regulares de $f$ é denso em $N$.

Sejam $M, N$ variedades diferenciáveis, $f: M \rightarrow N$ uma aplicação diferenciável e um ponto $p \in N$ vimos, no Teorema 1.1.14, que uma condição suficiente para que $f^{-1}(p)$ seja uma subvariedade de $M$ é que $p$ seja um valor regular de $f$. Deste fato é natural que façamos a seguinte pergunta: dada $S \subset N$ uma subvariedade de $N$, em que condições a imagem inversa $f^{-1}(S)$ é uma subvariedade de $M$ ? Uma resposta a esta questão é dada pela noção de transversalidade. Trata-se de uma generalização natural do conceito de valor regular.

Definição 1.1.16. Sejam $M$ uma variedade diferenciável $m$-dimensional, $N$ uma variedade diferenciável $n$-dimensional, $f: M \rightarrow N$ uma aplicação diferenciável e $P \subset N$ uma subvariedade $p$-dimensional de $N$, dizemos que $f$ é transversal a $P$ no ponto $q \in f^{-1}(P)$ quando

$$
d f_{q}\left(T_{q} M\right)+T_{f(q)} P=T_{f(q)} N
$$


ou seja, quando a imagem de $d f_{q}$ junto com o espaço tangente a $P$ em $f(q)$ geram todo $T_{f(q)} N$. Dizemos ainda que $f$ é transversal a $P$ se, para todo ponto $q \in f^{-1}(P) f$ é transversal a $P$ em $q$.

Teorema 1.1.17. Sejam $M$ uma variedade diferenciável $m$-dimensional, $N$ uma variedade diferenciável $n$-dimensional, $f: M \rightarrow N$ uma aplicação diferenciável e $P \subset N$ uma subvariedade $p$-dimensional de $N$. Se a aplicação $f$ é transversal a subvariedade $P$ então, ou bem $f^{-1}(P)=\varnothing$ ou bem $f^{-1}(P)$ é uma subvariedade $(m-n+p)$-dimensional de $M$.

Corolário 1.1.18. Seja $f: M \rightarrow N$ uma submersão, então para toda variedade $P \subset N$ ou bem $f^{-1}(P)=\varnothing$ ou bem $f^{-1}(P)$ é uma subvariedade de $M$.

O conceito de transversalidade é de grande importância, tendo várias aplicações. Como, por exemplo, o teorema que apresentaremos a seguir.

Teorema 1.1.19. Sejam $M$ uma variedade diferenciável $m$-dimensional e $f: M \rightarrow \mathbb{R}^{m}$ uma aplicação diferenciável. Consideremos um ponto $a \in \mathbb{R}^{m}$, um vetor $\vec{v} \neq \overrightarrow{0}$ em $\mathbb{R}^{m}$, e a reta $\Delta_{a}=\left\{a+t v: t \in \mathbb{R}^{m}\right\}$, que é uma subvariedade de $\mathbb{R}^{m}$. Definamos a aplicação $\tilde{f}: M \times \mathbb{R} \rightarrow \mathbb{R}^{m}$, dada por

$$
\tilde{f}(p, t)=f(p)+t v
$$

Nessas condições, f é transversal a $\Delta_{a}$ se, e somente se, $a \in \mathbb{R}^{m}$ é valor regular de $\tilde{f}$.

Demonstração: Seja $\varphi: V \rightarrow U$ uma carta de $M$, se $p \in V$, representaremos por $[J f(p)]_{m \times m}$ a matriz jacobiana de $f$, calculada no ponto $p$. As colunas de $[J f(p)]$ são os vetores imagens, pela aplicação linear $d f_{p}$, dos vetores da base de $T_{p} M$ associada a carta $\varphi$.

Acrescentemos à direita da matriz $[J f(p)]$ a coluna formada pelas componentes do vetor $\vec{v}$ de $\mathbb{R}^{m}$, e indiquemos a matriz assim obtida com o símbolo $[J f(p), \vec{v}]_{m \times m+1}$. Observemos que $[J f(p), \vec{v}]$ é exatamente a matriz jacobiana da aplicação $\tilde{f}$, calculada no ponto $(p, t) \in M \times \mathbb{R}$. Assim, se $f$ é transversal a $\Delta_{a}$ para todo $p \in M$, tal que $f(p) \in \Delta_{a}$, então

$$
d f_{p}\left(T_{p} M\right)+T_{f(p)} \Delta_{a}=\mathbb{R}^{m}
$$

e notemos que $T_{f(p)} \Delta_{a}=\{t v: t \in \mathbb{R}\}$. 
Mas, dado um ponto $(p, h) \in M \times \mathbb{R}$, temos

$$
d \tilde{f}_{(p, h)}\left(T_{p} M \times \mathbb{R}\right)=d f_{p}\left(T_{p} M\right)+t v, \quad \text { onde } t \in \mathbb{R} .
$$

Da condição de transversalidade, temos que $d \tilde{f}_{(p, h)}\left(T_{p} M \times \mathbb{R}\right)=d f_{p}\left(T_{p} M\right)+t v=\mathbb{R}^{m}$, onde $t \in \mathbb{R}$, daí decorre que a matriz $[J f(p), \vec{v}]$ tem posto igual $m$. Por fim notemos que, se $p \in M$ é tal que $\tilde{f}(p, t)=a$, para algum $t \in \mathbb{R}$, então $\tilde{f}(p, t)=f(p)+t \vec{v}=a$, o que implica que $f(p) \in \Delta_{a}$. Logo, o ponto $a \in \mathbb{R}^{m}$ é um valor regular de $\tilde{f}$.

Por outro lado, suponhamos que o ponto $a \in \mathbb{R}^{m}$ é valor regular de $\tilde{f}$, então para todo ponto $(p, t) \in \tilde{f}^{-1}(a)$, temos $d \tilde{f}_{(p, t)}\left(T_{p} M \times \mathbb{R}\right)=d f_{p}\left(T_{p} M\right)+t v=\mathbb{R}^{m}$. Mas, se o ponto $(p, t)$ pertence a $\tilde{f}^{-1}(a)$ significa que $f(p)=a-t v$, ou seja, que $f(p) \in \Delta_{a}$. Portanto, $f$ é transversal a $\Delta_{a}$.

Dada uma aplicação diferenciável $f: M \rightarrow N$, no Capítulo 3, definiremos um número, chamado o grau de $f$, para tanto precisaremos do conceito de variedades orientadas, que apresentaremos a seguir.

Começaremos este estudo falando de orientação em espaços vetoriais. Então, usaremos a definição de orientação sobre espaços vetoriais e o conceito de espaço tangente para definirmos orientação sobre variedades.

Suponha que $V$ é um espaço vetorial real de dimensão finita e $\beta=\left\{v_{1}, \ldots, v_{n}\right\}$ é uma base ordenada de $V$. Se $\beta^{\prime}=\left\{v_{1}^{\prime}, \ldots, v_{n}^{\prime}\right\}$ é outra base ordenada de $V$, então existe um único isomorfismo $I: V \rightarrow V$, tal que $I(\beta)=\beta^{\prime}$, a saber

$$
I\left(\alpha_{1} v_{1}+\ldots+\alpha_{n} v_{n}\right)=\alpha_{1} v_{1}^{\prime}+\ldots+\alpha_{n} v_{n}^{\prime}
$$

Dizemos que $\beta$ e $\beta^{\prime}$ tem orientação equivalente se o determinante da transformação linear $I$ é positivo. Isto é, se escrevemos

$$
\begin{gathered}
I\left(v_{1}\right)=v_{1}^{\prime}=a_{11} v_{1}+\ldots+a_{n 1} v_{n} \\
\vdots \\
I\left(v_{n}\right)=v_{n}^{\prime}=a_{1 n} v_{1}+\ldots+a_{n n} v_{n} .
\end{gathered}
$$


então, $\beta$ e $\beta^{\prime}$ têm orientação equivalente se $\operatorname{det}[I]_{\beta}>0$, onde

$$
[I]_{\beta}=\left[\begin{array}{ccc}
a_{11} & \cdots & a_{1 n} \\
\vdots & \ddots & \vdots \\
a_{n 1} & \cdots & a_{n n}
\end{array}\right]
$$

Observemos que pelo fato de $I$ ser um isomorfismo, $\operatorname{det}[I]_{\beta} \neq 0$. Além disso, notemos que $[I]_{\beta}=M_{\beta}^{\beta^{\prime}}$, ou seja, a matriz da transformação $I$ com relação a base $\beta$ é igual a matriz de mudança de base $\beta$ para $\beta^{\prime}$. Então, graças a regra do produto para determinantes e lembrando que

$$
M_{\beta}^{\beta^{\prime}}=M_{\beta^{\prime}}^{\beta^{\prime \prime}} M_{\beta}^{\beta^{\prime}}
$$

temos que dadas bases $\beta, \beta^{\prime}$ e $\beta^{\prime \prime}$ de $V$ e $I_{1}, I_{2}$ os isomorfismos de $V$ em $V$, tais que $I_{1}(\beta)=\beta^{\prime}$ e $I_{2}\left(\beta^{\prime}\right)=\beta^{\prime \prime}$, temos

$$
\left[I_{2} \circ I_{1}\right]=M_{\beta}^{\beta^{\prime \prime}}=M_{\beta^{\prime}}^{\beta^{\prime \prime}} M_{\beta}^{\beta^{\prime}},
$$

$\log 0 \operatorname{det}\left[I_{2} \circ I_{1}\right]_{\beta}=\operatorname{det}\left[I_{2}\right]_{\beta^{\prime}} \operatorname{det}\left[I_{1}\right]_{\beta}$.

Assim, este processo define uma relação de equivalência, particionando o conjunto de todas as bases ordenadas de $V$ em duas classes.

Definição 1.1.20. Uma orientação de $V$ é uma decisão arbitrária para fixar um sinal positivo para elementos de uma classe de equivalência e um sinal negativo para os outros. Um espaço vetorial orientado $V$ é um par formado pelo espaço vetorial $V$ e uma orientação de $V$. As bases de $V$ pertencentes a esta orientação serão chamadas de positivas e as outras de negativas.

Exemplo 1.1.21. Por exemplo, o espaço euclidiano $\mathbb{R}^{n}$ será sempre orientado positivamente pela base canônica. 


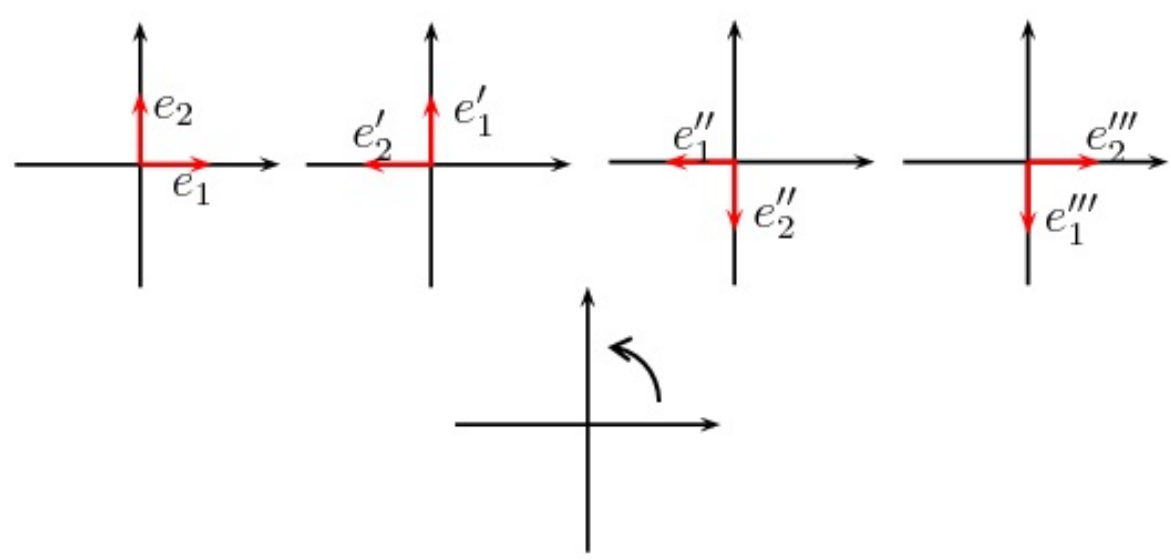

Figura 1.2: Exemplo de orientação em $\mathbb{R}^{2}$

Observação 1.1.22. Dado $V$ um espaço vetorial real de dimensão finita, quando falamos de uma orientação de $V$ é indispensável considerarmos as suas bases ordenadas. Por exemplo, se considerarmos $\beta=\left\{e_{1}, e_{2}\right\}$ como sendo uma base positiva de $\mathbb{R}^{2}$, onde $e_{1}=$ $(1,0)$ e $e_{2}=(0,1)$, então a base $\beta^{\prime}=\left\{e_{1}^{\prime}, e_{2}^{\prime}\right\}$, com $e_{1}^{\prime}=(0,1)$ e $e_{2}^{\prime}=(-1,0)$, é equivalente a $\beta$, porém a base $\alpha=\left\{f_{1}, f_{2}\right\}$, com $f_{1}=(-1,0)$ e $f_{2}=(0,1)$, não é equivalente a base $\beta$.

Dados $V$ e $W$ dois espaços vetoriais de mesma dimensão $n$, se $I: V \rightarrow W$ é um isomorfismo de $V$ em $W$ sabemos que $I$ leva uma base de $V$ em uma base de $W$. Assim, temos a seguinte definição.

Definição 1.1.23. Sejam $V$ e $W$ dois espaços vetoriais $n$-dimensionais e orientados, se $I: V \rightarrow W$ é um isomorfismo de $V$ em $W$, dizemos que $I$ preserva orintação se $I$ leva base positiva de $V$ em uma base positiva de $W$, ou equivalentemente se $I$ leva base negativa de $V$ em uma base negativa de $W$. Caso contrário, diremos que $I$ inverte orientação. Quando um isomorfismo entre espaços vetoriais orientados preserva (inverte) orientação, dizemos também que esse isomorfismo é positivo (negativo).

Agora, voltemos para variedades.

Definição 1.1.24. Seja $M$ uma variedade diferenciável $m$-dimensional, dizemos que $M$ é orientável se existe uma escolha contínua de orientações para todos os espaços tangentes $T_{p} M$. A condição de continuidade pode ser interpretada no seguinte sentido: próximo de 
cada ponto $p \in M$ deve existir uma carta $\varphi: U \rightarrow V$, com $p \in U$, tal que $d \varphi_{u}: T_{u} M \rightarrow \mathbb{R}^{m}$ preserva orientação em cada ponto $u \in U$. Uma variedade orientada é um par $(M, \mathcal{O})$ onde $M$ é uma variedade diferenciável e $\mathcal{O}$ é uma orientação em $M$.

Exemplo 1.1.25. A circunferência unitária $\mathbb{S}^{n} \subset \mathbb{R}^{n+1}$ é orientável.

Exemplo 1.1.26. Dada uma variedade diferenciável $M$, o fibrado tangente $T M$ de $M$ é uma variedade diferenciável orientável, quer $M$ o seja, quer não.

Observação 1.1.27. Dada uma variedade diferenciável $M m$-dimensional, já vimos que para cada ponto $p \in M$ obtemos o espaço vetorial $T_{p} M$. Dada $\varphi: V \rightarrow U$ uma carta de $M$ com $p \in V$, denotaremos por $\left\{\frac{\partial}{\partial x_{1}}(p), \ldots, \frac{\partial}{\partial x_{m}}(p)\right\}$ a base $\left\{d \varphi_{\varphi(p)}^{-1}\left(e_{1}\right), \ldots, d \varphi_{\varphi(p)}^{-1}\left(e_{m}\right)\right\}$ de $T_{p} M$, onde $\left\{e_{1}, \ldots, e_{m}\right\}$ denota a base canônica de $\mathbb{R}^{m}$ e $\varphi(p)=\left(x_{1}, \ldots, x_{m}\right) \in \mathbb{R}^{m}$. Além disso, consideraremos o espaço vetorial $T_{p} M$ positivamente orientado pela base $\left\{\frac{\partial}{\partial x_{1}}(p), \ldots, \frac{\partial}{\partial x_{m}}(p)\right\}$, salvo o caso em que digamos o cantrário.

Definição 1.1.28. Sejam $M, N$ variedades diferenciáveis $n$-dimensionais, orientadas e $f: M \rightarrow N$ uma aplicação diferenciável. Dados um valor regular $p \in N$ de $f$ e os pontos regulares $q \in f^{-1}(p)$ diremos que o ponto $q$ é positivo $(q>0)$ se o isomorfismo $d f_{q}: T_{q} M \rightarrow T_{p} N$ preserva a orientação e diremos que o ponto $q$ é negativo $(q<0)$ se o isomorfismo $d f_{q}: T_{q} M \rightarrow T_{p} N$ inverte a orientação.

Teorema 1.1.29. Sejam $M$ e $N$ variedades diferenciáveis $m$-dimensionais orientadas, com $M$ conexa. Se $f: M \rightarrow N$ é um difeomorfismo local, então $d f_{x}: T_{x} M \rightarrow T_{f(x)} N$ é positivo ou é negativo para todo $x \in M$.

Apresentaremos a seguir, o conceito de variedades diferenciáveis com bordo. Uma referêcia deste assunto é [4].

Definição 1.1.30. Uma variedade diferenciável $m$-dimensional com bordo é um espaço topológico $M$ com uma coleção de aplicações $\varphi: V \rightarrow U$, onde $V$ é um aberto de $M$ e $U$ é um aberto do subconjunto $\mathbb{H}^{m}=\left\{\left(x_{1}, \ldots, x_{m}\right): x_{m} \geq 0\right\}$, satisfazendo as condições da definição de variedades diferenciáveis (ver Definição 1.1.7).

Podemos definir também o conceito de variedades complexas. Uma referência deste assunto é [20]. 
Definição 1.1.31. Dizemos que uma variedade diferenciável $M$ de dimensão $2 m$ é uma variedade complexa de dimensão $m$, se $M$ tem um atlas formado apenas por cartas em $\mathbb{C}^{m}$, tais que a mudança de coordenadas entre estas cartas seja holomorfa.

Dado que aplicações holomorfas são muito mais especiais que as aplicações diferenciáveis, temos que a teoria de variedades diferenciáveis e variedades complexas têm aspectos muito diferentes. O teorema a seguir serve de ilustração para este fato.

Teorema 1.1.32. Toda variedade complexa $M$ é orientável.

A demonstração do teorema acima pode ser vista em [20].

\subsection{Homotopia}

Nesta seção introduzimos noções básicas sobre homotopia, procurando ilustrá-las com alguns exemplos.

Definição 1.2.1. Sejam $X$ e $Y$ espaços topológicos, dizemos que duas aplicações contínuas são homotópicas quando existe uma aplicação contínua

$$
H: X \times I \rightarrow Y, \quad \text { com } I=[0,1]
$$

tal que $H(x, 0)=f(x)$ e $H(x, 1)=g(x)$ para todo $x \in X$. Chamaremos a aplicação $H$ de homotopia entre $f$ e $g$ e escreveremos $f \simeq g$.

Exemplo 1.2.2. Sejam $E$ um espaço vetorial normado, $Y \subset E$ um espaço topológico com a topologia induzida de $E$ e $X$ um espaço topológico qualquer. Dadas aplicações contínuas $f, g: X \rightarrow Y$, suponhamos que, para todo $x \in X$, o segmento de reta $[f(x), g(x)]$ esteja contido em $Y$. Então, $f \simeq g$.

De fato, denotando o intervalo $[0,1]$ por $I$, basta definirmos $H(x, t)=(1-t) f(x)+t g(x)$ para obtermos uma homotopia $H: X \times Y \rightarrow Y$ entre $f$ e $g$.

Em particular, dada uma aplicação constante

$$
\begin{gathered}
f: X \quad \longrightarrow \quad E \\
x \longmapsto c
\end{gathered}
$$


toda aplicação contínua $g: X \rightarrow E$ é homotópica a $f$.

Exemplo 1.2.3. Seja $\mathbb{S}^{n}$ a esfera unitária de $\mathbb{R}^{n+1}$, dadas duas aplicações contínuas $f, g: X \rightarrow \mathbb{S}^{n}$, se $f(x) \neq-g(x)$ para todo $x \in X$, isto é, se $f(x)$ e $g(x)$ nunca são pontos antípodas, então $f \simeq g$.

Proposição 1.2.4. Sejam $X, Y$ e $Z$ espaços topológicos, consideremos aplicações $f, f^{\prime}$ : $X \rightarrow Y$ e $g, g^{\prime}: Y \rightarrow Z$ aplicações contínuas. Se $f \simeq f^{\prime}$ e $g \simeq g^{\prime}$, então $g \circ f \simeq g^{\prime} \circ f^{\prime}$. Demonstração: Seja $I=[0,1]$, denotemos por $H: X \times I \rightarrow Y$ uma homotopia entre $f$ e $f^{\prime}$ e por $K: Y \times I \rightarrow Z$ uma homotopia entre $g$ e $g^{\prime}$. Definindo $L: X \times I \rightarrow Z$ por $L(x, t)=K(H(x, t), t)$, temos que $L$ é uma homotopia entre $g \circ f$ e $g^{\prime} \circ f^{\prime}$.

Existe uma estreita relação entre homotopia e o problema de estender continuamente a todo o espaço uma aplicação contínua definida num subconjunto fechado desse espaço. Um exemplo disto pode ser visto na seguinte Proposição.

Proposição 1.2.5. Sejam $X$ um espaço topológico e $\overline{\mathbb{B}}_{1}(0)$ a bola fechada de centro 0 e raio 1 de $\mathbb{R}^{n+1}$. Uma aplicação contínua $f: \mathbb{S}^{n} \rightarrow X$ estende-se continuamente à $\overline{\mathbb{B}}_{1}(0)$ se, e somente se, é homotópica a uma constante.

Demonstração: Denotemos por $I$ o intervalo $[0,1]$ e consideremos a aplicação

$$
\begin{aligned}
\varphi: \mathbb{S}^{n} \times I & \longrightarrow \overline{\mathbb{B}}_{1}(0) \\
(x, t) & \longmapsto(1-t) x
\end{aligned}
$$

que é contínua, sobrejetora e igual a 0 em $\mathbb{S}^{n} \times 1$. Se $\tilde{f}: \overline{\mathbb{B}}_{1}(0) \rightarrow X$ é uma extensão contínua de $f: \mathbb{S}^{n} \rightarrow X$, então

$$
H=\tilde{f} \circ \varphi: \mathbb{S}^{n} \times I \rightarrow X
$$

é uma homotopia entre $f$ e a aplicação constante $g: \mathbb{S}^{n} \rightarrow X$, definida por $g(x)=\tilde{f}(0)$. Reciprocamente, suponhamos que $H: \mathbb{S}^{n} \rightarrow X$ seja uma homotopia entre $f$ e uma aplicação constante $g: \mathbb{S}^{n} \rightarrow X$, dada por $g(x)=p$, para todo $x \in \mathbb{S}^{n}$. Seja a aplicação 
$\tilde{f}: \mathbb{S}^{n} \rightarrow X$ definida da seguinte maneira,

$$
\tilde{f}(x)=\left\{\begin{array}{l}
H\left(\frac{x}{|x|}, 1-|x|\right), \text { se } \quad x \neq 0 \\
p, \text { se } x=0
\end{array} .\right.
$$

Assim, temos que $\tilde{f}$ é contínua e $\left.\tilde{f}\right|_{\mathbb{S}^{n}}=f$.

A seguir apresentamos um resultado, cuja demonstração pode ser vista em [16], e que nos será indispensável na definição do índice de Poincaré-Hopf para singularidades de campos de vetores contínuos.

Teorema 1.2.6. Sejam $M$ e $N$ variedades diferenciáveis compactas, então toda aplicação contínua $f: M \rightarrow N$ é homotópica a uma aplicação $g: M \rightarrow N$ de classe $C^{1}$.

\subsection{Homologia singular}

Nesta seção apresentamos um estudo da teoria de homologia singular. Faremos o estudo dos R-módulos de homologia singular com coeficientes em um anel R comutativo com identidade. Uma referência para este assunto é [21].

\section{Simplexos em $\mathbb{R}^{n}$}

Definição 1.3.1. Dados $(p+1)$ pontos em $\mathbb{R}^{n}, p \leq n$, dizemos que $\left\{X_{0}, \ldots, X_{p}\right\}$ é uma coleção geometricamente independente se os vetores $\overrightarrow{v_{1}}=X_{1}-X_{0}, \overrightarrow{v_{2}}=X_{2}-X_{0}, \ldots$, $\overrightarrow{v_{p}}=X_{p}-X_{0}$ são linearmente independentes.

Definição 1.3.2. Seja $\left\{X_{0}, \ldots, X_{p}\right\} \subset \mathbb{R}^{n}$ uma coleção geometricamente independente de $\mathbb{R}^{n}, p \leq n$. O $p$-simplexo $S$ gerado por $X_{0}, \ldots, X_{p}$ é a envoltória convexa:

$$
S=E C\left(\left\{X_{0}, \ldots, X_{p}\right\}\right)
$$

Os pontos $X_{i}$ são chamados vértices do $p$-simplexo $\mathrm{S}$.

Teorema 1.3.3. (Teorema das coordenadas baricêntricas) Sejam $\left\{X_{0}, \ldots, X_{p}\right\}$ uma coleção de $(p+1)$ pontos geometricamente independentes em $\mathbb{R}^{n}$, com $p \leq n$. Considere 
o seguinte conjunto:

$$
A_{p}=\left\{t_{0} X_{0}+\ldots+t_{p} X_{p} ; \quad 0 \leq t \leq 1 \text { e } \sum_{i=0}^{p} t_{i}=1\right\} \subset \mathbb{R}^{n}
$$

(i) $A_{p}=E C\left(\left\{X_{0}, \ldots, X_{p}\right\}\right)=p$-simplexo gerado por $X_{0}, \ldots, X_{p}$;

(ii) $\operatorname{Se} \sum_{i=0}^{p} t_{i} X_{i}=\sum_{i=0}^{p} s_{i} X_{i}, \quad 0 \leq t_{i}, s_{i} \leq 1 \mathrm{com} \sum_{i=0}^{p} t_{i}=\sum_{i=0}^{p} s_{i}=1$, então $t_{i}=s_{i}$ para todo $i=1, \ldots, p$, ou seja: todo elemento de $A_{p}$ se escreve de maneira única como combinação linear dos pontos $\left\{X_{0}, \ldots, X_{p}\right\}$.

Observação 1.3.4. Concluímos que escolhida uma ordenação $\left\{X_{0}, \ldots, X_{p}\right\}$ para $(p+1)$ pontos geometricamente independentes em $\mathbb{R}^{n}$, com $p \leq n$, cada ponto $X \in E C\left(\left\{X_{0}, \ldots, X_{p}\right\}\right)$ é univocamente determinado por uma $(p+1)$-upla $\left(t_{0}, t_{1}, \ldots, t_{p}\right)$, com $0 \leq 1$ e $\sum_{i=0}^{p} t_{i}=1$ denominada as coordenadas baricêntricas do ponto $X$.

Definição 1.3.5. Sejam $X_{0}=e_{1}=(1,0, \ldots, 0), X_{1}=e_{2}=(0,1,0, \ldots, 0), \ldots, X_{p}=$ $e_{p+1}=(0,0, \ldots, 0,1)$. O $p$-simplexo $E C\left(\left\{X_{0}, \ldots, X_{p}\right\}\right)$, nesse caso, é denominado $p$-simplexo padrão e será denotado por $\Delta_{p} \in \mathbb{R}^{p+1}$

\section{Homologia singular de um espaço topológico $X$ com coeficientes em um anel $R$}

Fixemos $R$ um anel comutativo com a identidade $1_{R}$ e consideremos $\Delta_{p} \subset \mathbb{R}^{p+1} \mathrm{o}$ $p$-simplexo padrão munido da topologia induzida de subespaço em $\mathbb{R}^{p+1}$ (munido da topologia usual).

Definição 1.3.6. Para cada espaço topológico $X$, um $p$-simplexo singular em $X$ é uma função contínua $\sigma: \Delta_{p} \rightarrow X$.

Denotaremos por

$$
C_{p}(X)=\left\{\sigma: \Delta_{p} \rightarrow X ; \quad \sigma \text { é contínua }\right\}
$$

o conjunto de todos os $p$-simplexos singulares de $X$. 
Definição 1.3.7. Para cada $p \geq 0$, definimos o $R$-módulo livre cuja base é o conjunto de todos os $p$-simplexos singulares de $X$ :

$S_{p}\left(C_{p}(X), R\right)=\left\{f: C_{p}(X) \rightarrow R ; f(\sigma) \neq 0\right.$, apenas para um n finito de elementos $\left.\sigma\right\}$

Um elemento típico de $S_{p}\left(C_{p}(X), R\right)$ é uma combinação linear formal $\alpha_{1} \sigma_{1}+\ldots+\alpha_{r} \sigma_{r}$, onde $\alpha_{i} \in R$ e $\sigma_{i}: \Delta_{p} \rightarrow X$ é um $p$-simplexo singular de $X$.

$$
\begin{aligned}
\sum_{i=0}^{r} \alpha_{i} \sigma_{i} \longleftrightarrow f: C_{p}(X) & \rightarrow R \\
\sigma & \mapsto f(\sigma)= \begin{cases}\alpha_{i} ; & \text { se } \sigma=\sigma_{i} i=1, \ldots, r \\
0, & \text { se } \sigma \neq \sigma_{i}\end{cases}
\end{aligned}
$$

Os elementos de $S_{p}\left(C_{p}(X) ; R\right)$ são chamados p-cadeias singulares de $\mathbf{X}$ com coeficientes em $\mathbf{R}$.

A seguir, definiremos o complexo de cadeias $\left\{S_{p}(X ; R) ; \partial\right\}_{p \geq 0}$. Para isso, convencionaremos que $\left\{S_{p}(X ; R)\right\}=\{0\}$ para todo $p<0$.

\section{Os operadores face}

Definição 1.3.8. Seja $\sigma: \Delta_{p} \rightarrow X$ um $p$-simplexo singular de $X$. Considere a inclusão

$$
\begin{array}{ccc}
\Delta_{p-1} & \stackrel{\varepsilon_{i}}{\hookrightarrow} & \Delta_{p} \\
\left(t_{0}, \ldots, t_{i}, \ldots, t_{p-1}\right) & \hookrightarrow & \left(t_{0}, \ldots, t_{i-1}, 0, t_{i}, \ldots, t_{p-1}\right),
\end{array}
$$

que insere o zero na i-ésima posição. Para cada $i=1, \ldots, p$ definimos a i-ésima face de $\sigma$ como sendo o $(p-1)$-simplexo singular de $X$ dado pela composição

$$
\sigma \circ \varepsilon_{i}: \Delta_{p-1} \rightarrow X
$$

que é contínua e denotada por $\partial_{i} \sigma: \Delta_{p-1} \rightarrow X$. 
Assim, para cada $i=1, \ldots, p$ e para cada $p$-simplexo singular $\sigma \in C_{p}(X)$, associamos a sua i-ésima face $\partial_{i} \sigma \in C_{p-1}(X)$. Isso define uma função

$$
\begin{array}{ccc}
\partial_{i}: C_{p}(X) & \longrightarrow & C_{p-1}(X) \subset S_{p-1}(X ; R) \\
\sigma & \longmapsto & \partial_{i} \sigma
\end{array}
$$

para todo $i=1, \ldots, p$, e desde que $C_{p}(X)$ é base para o R-módulo livre $S_{p}(X ; R), \partial_{i}$ se estende por linearidade a um único R-homomorfismo $\partial_{i}: S_{p}(X ; R) \rightarrow S_{p-1}(X ; R)$, chamado operador face. O R-homomorfismo $\partial: S_{p}(X ; R) \rightarrow S_{p-1}(X ; R)$ dado pela soma alternada dos operadores face

$$
\partial=\partial_{0}-\partial_{1}+\partial_{2}-\ldots+(-1)^{p} \partial_{p}=\sum_{i=0}^{p}(-1)^{i} \partial_{i}
$$

é chamado operador bordo.

Teorema 1.3.9. O operador $\partial: S_{p}(X ; R) \rightarrow S_{p-1}(X ; R)$, definido por $\sum_{i=0}^{p}(-1)^{i} \partial_{i}$, é tal que $\partial \circ \partial=0$.

Definição 1.3.10. Fixado um anel comutativo com identidade $R$ para cada espaço topológico $X$, criamos um complexo de cadeias $S_{*}(X ; R)=\left\{S_{p}(X ; R), \partial\right\}_{p \geq 0}$, ou seja,

$$
S_{*}(X ; R): \cdots \stackrel{\partial}{\longrightarrow} S_{p}(X ; R) \stackrel{\partial}{\longrightarrow} S_{p-1}(X ; R) \stackrel{\partial}{\longrightarrow} \cdots \stackrel{\partial}{\longrightarrow} S_{1}(X ; R) \stackrel{\partial}{\longrightarrow} S_{0}(X ; R) \stackrel{\partial}{\longrightarrow} 0,
$$

usaremos as seguintes terminologias:

$Z_{p}\left(S_{*}(X ; R)\right) \doteq Z_{p}(X ; R)=\operatorname{Ker}(\partial)$ o submódulo dos p-ciclos,

$B_{p}\left(S_{*}(X ; R)\right) \doteq B_{p}(X ; R)=\operatorname{Im}(\partial)$ o submódulo dos p-bordos e

$H_{p}\left(S_{*}(X ; R)\right) \doteq H_{p}(X ; R)=\frac{Z_{p}(X ; R)}{B_{p}(X ; R)}$ o p-ésimo R-módulo de homologia de $X$ com coeficientes em $\mathrm{R}$.

Um elemento típico de $H_{p}(X ; R)$ é da forma

$$
\mu=z_{p}+B_{p}(X ; R)
$$


onde $z_{p} \in Z_{p}(X ; R)$ é um p-ciclo; $\mu$ é chamada classe de homologia de $\mathbf{X}$ com coeficientes em $\mathbf{R}$, representada pelo p-ciclo $z_{p}$.

\section{Construção do R-homomorfismo induzido em homologia}

Dada uma função contínua $f: X \rightarrow Y$, queremos definir um $R$-homomorfismo $f_{*}: H_{p}(X ; R) \rightarrow H_{p}(Y ; R)$ para cada $p \geq 0$. Para isso, precisamos definir uma aplicação de cadeias

$$
f_{\#}: S_{p}(X ; R) \rightarrow S_{p}(Y ; R)
$$

para cada $p \geq 0$, entre os complexos de cadeias $S_{*}(X ; R)$ e $S_{*}(Y ; R)$.

Observemos que, dado um $p$-simplexo singular de $X, \sigma: \Delta_{p} \rightarrow X$, a composição

$$
f \circ \sigma: \Delta_{p} \stackrel{\sigma}{\longrightarrow} X \stackrel{f}{\longrightarrow} Y
$$

é um $p$-simplexo singular de $Y$ e determina uma função

$$
\begin{aligned}
C_{p}(X) & \longrightarrow C_{p}(Y) \quad \subset S_{p}(Y ; R) \\
\sigma & \longmapsto f \circ \sigma
\end{aligned}
$$

para cada $p \geq 0$. Desde que $C_{p}(X)$ é base para o $R$-módulo livre $S_{p}(X ; R)$ e $C_{p}(Y) \subset S_{p}(Y ; R)$ tal função se estende por linearidade a um único $R$-homomorfismo $f_{\#}: S_{p}(X ; R) \rightarrow S_{p}(Y ; R)$, definido por

$$
f_{\#}\left(\alpha_{1} \sigma_{1}+\ldots+\alpha_{r} \sigma_{r}\right)=\alpha_{1}\left(f \circ \sigma_{1}\right)+\ldots+\alpha_{r}\left(f \circ \sigma_{r}\right)
$$

Teorema 1.3.11. O $R$-homomorfismo $f_{\#}: S_{p}(X ; R) \rightarrow S_{p}(Y ; R)$ é uma aplicação de cadeias.

Portanto, $f_{\#}: S_{p}(X ; R) \rightarrow S_{p}(Y ; R)$ induz um bem definido $R$-homomorfismo $f_{*}: H_{p}(X ; R) \rightarrow H_{p}(Y ; R)$, dado por

$$
f_{*}\left(z_{p}\right)+B_{p}(X ; R)=f_{\#}\left(z_{p}\right)+B_{p}(Y ; R),
$$


onde

$$
f_{\#}\left(z_{p}\right)=f_{\#}\left(\alpha_{1} \sigma_{1}+\ldots+\alpha_{r} \sigma_{r}\right)=\alpha_{1}\left(f \circ \sigma_{1}\right)+\ldots+\alpha_{r}\left(f \circ \sigma_{r}\right) .
$$

\subsection{Teoria de singularidades}

Nesta seção, apresentamos as definições e principais resultados da teoria de singularidades que serão usados para o desenvolvimento deste trabalho. Para o leitor interessado recomendamos [6, 19, 27].

Um dos nossos objetivos é estudar localmente funções analíticas $f: \mathbb{C}^{n+k} \rightarrow \mathbb{C}^{k}$. Para isso, introduzimos uma relação de equivalência no espaço das funções analíticas definidas num aberto contendo a origem de $\mathbb{C}^{n}$, da seguinte forma:

Definição 1.4.1. Dizemos que a função $f: \mathbb{C}^{n} \rightarrow \mathbb{C}$ é equivalente à função $g: \mathbb{C}^{n} \rightarrow \mathbb{C}$, se existe um aberto $U \subset \mathbb{C}^{n}$, com $0 \in U$, tal que $f_{\mid U} \equiv g_{\mid U}$. As classes de equivalência são chamadas de germes, denotadas por $f:\left(\mathbb{C}^{n}, 0\right) \rightarrow \mathbb{C}$, ou simplesmente, por $f$. A coleção de todos esses germes de funções citados acima é denotada por $\mathcal{O}_{n}$. Observemos que $\mathcal{O}_{n}$ é um anel noetheriano local cujo ideal maximal é dado por $\mathcal{M}_{n}=\left\{f \in \mathcal{O}_{n}: f(0)=0\right\}$.

Definição 1.4.2. Denotamos por $\mathcal{O}_{n, p}$ o conjunto dos germes de aplicações analíticas $f:\left(\mathbb{C}^{n}, 0\right) \rightarrow \mathbb{C}^{p}$ e por $\mathcal{O}_{n, p}^{0}$ o conjunto dos germes $f:\left(\mathbb{C}^{n}, 0\right) \rightarrow\left(\mathbb{C}^{p}, 0\right)$.

Proposição 1.4.3. $\mathcal{O}_{n, p}$ é um $\mathcal{O}_{n}$-módulo livre de posto $p$.

O teorema de fibração de Milnor, descrito em [19] é um dos resultados centrais na teoria de singularidades, tendo generalizações em diversas situações.

Teorema 1.4.4 ([19]). Seja $f:\left(\mathbb{C}^{n}, 0\right) \rightarrow(\mathbb{C}, 0)$ germe de aplicação analítica, $V=f^{-1}(0)$, $\mathbb{B}_{\varepsilon}$ bola centrada na origem e de raio $\varepsilon$ e $\mathbf{K}=V \cap \mathbb{S}^{2 n-1}$. Se $\varepsilon$ for suficientemente pequeno temos que $V \cap \mathbb{B}_{\varepsilon}$ é homeomorfo ao cone $C(\mathbf{K})=\{t z: 0 \leq t \leq 1, z \in \mathbf{K}\}$.

Seja $\phi: \mathbb{S}_{\varepsilon} \backslash \mathbf{K} \rightarrow \mathbb{S}^{1}$ dada por $\phi(z)=\frac{f(z)}{|f(z)|}$, e denotemos $\phi^{-1}\left(e^{i \theta}\right)=\mathbf{F}_{\theta}$.

O resultado básico da teoria de Milnor é o teorema da fibração, que mostra que $\phi$ define uma fibração de $\mathbb{S}_{\varepsilon} \backslash \mathbf{K} \rightarrow \mathbb{S}^{1}$ com fibra $\mathbf{F}_{\theta}$. 
Teorema 1.4.5 ([19]). Para $\varepsilon$ suficientemente pequeno temos que $\mathbb{S}_{\varepsilon} \backslash \mathbf{K}$ é um fibrado diferenciável localmente trivial sobre $\mathbb{S}^{1}$, com projeção $\phi$ e fibra $\mathbf{F}_{\theta}$ como acima.

Definição 1.4.6. $\mathbf{F}_{\theta}$ é dita a fibra de Milnor de $f$.

Em [19] Milnor provou que as fibras $\mathbf{F}_{\theta}$ são todas difeomorfas. Também em [19], Milnor apresentou uma descrição alternativa da fibra de Milnor.

Teorema 1.4.7. Sejam $c \in \mathbb{C}^{*}$ um número complexo suficientemente próximo de zero e $f:\left(\mathbb{C}^{n}, 0\right) \rightarrow(\mathbb{C}, 0)$ germe de aplicação analítica, então a interseção da hipersuperfície complexa $f^{-1}(c)$ com a bola aberta $\mathbb{B}_{\varepsilon}(0)$ de centro 0 e raio $\varepsilon$ de $\mathbb{C}^{n}$ é uma variedade suave difeomorfa a fibra $\mathbf{F}_{\theta}$.

Nas hipóteses do teorema acima, denotaremos $f^{-1}(c) \cap \mathbb{B}_{\varepsilon}(0)=\mathbf{F}_{f}$.

O número de Milnor, que definiremos a seguir, é um invariante de suma importância na teoria de singularidades, auxiliando na teoria de classificação.

Definição 1.4.8. Sejam $f \in \mathcal{O}_{n}$, germe de função analítica com singularidade isolada na origem, e $J(f)$ o ideal jacobiano de $f$. Definimos o número de Milnor $\mu$ como $\operatorname{dim}_{\mathbb{C}} \frac{\mathcal{O}_{n}}{J(f)}$.

Teorema 1.4.9. Seja $f \in \mathcal{O}_{n}$ germe de função analítica com singularidade isolada na origem, então cada fibra $\mathbf{F}_{f}$ tem o mesmo tipo de homotopia de um buquê de esferas $\mathbb{S}^{n-1}$, e o número de esferas do buquê é exatamente $\mu$.

A definição de número de Milnor que vimos acima foi introduzida por J. Milnor em [19] para o caso particular de hipersuperfícies, pórem existe uma definição mais geral para este invariante, para a qual precisaremos da noção de interseção completa.

\section{Interseção completa com singularidade isolada}

Seja $f: \mathbb{C}^{n+k} \rightarrow \mathbb{C}^{k}$ uma aplicação holomorfa com uma singularidade isolada em 0 e consideremos $V=f^{-1}(0)$.

Definição 1.4.10. Dizemos que $V$ é uma interseção completa geométrica, se é definida como o conjunto comum de zeros (germes de) $k$ funções holomorfas. Também, $V$ é uma interseção completa se o ideal $I(V)=\left\{g \in \mathcal{O}_{n+k}: g(x)=0, \forall x \in V\right\}$ é gerado por $k$ funções holomorfas. 
Proposição 1.4.11. Sejam $f: \mathbb{C}^{n+k} \rightarrow \mathbb{C}^{k}$ uma aplicação holomorfa com uma singularidade isolada em 0 e $V=f^{-1}(0)$. Então, $V$ é uma interseção completa geométrica se, e somente se, é uma interseção completa.

Abreviaremos uma interseção completa com singularidade isolada, por ICSI.

A seguir apresentamos o número de Milnor para uma ICSI, definido em [11] por H. Hamm.

Teorema 1.4.12 ([11]). Seja $f=\left(f_{1}, \ldots, f_{k}\right): \mathbb{C}^{n+k} \rightarrow \mathbb{C}^{k}$ uma aplicação (germe) analítica com singularidade isolada na origem, e $V=f^{-1}(0)$ uma ICIS. Cada fibra $\mathbf{F}_{f}$ tem o mesmo tipo de homotopia de um buquê de esferas $\mathbb{S}^{n}$.

Definição 1.4.13. Seja $f=\left(f_{1}, \ldots, f_{k}\right): \mathbb{C}^{n+k} \rightarrow \mathbb{C}^{k}$ uma aplicação (germe) analítica com singularidade isolada na origem, $V=f^{-1}(0)$ uma ICIS e $c \in \mathbb{C}^{k}$ suficientemente próximo de $0 \in \mathbb{C}^{k}$. Dada uma fibra $\mathbf{F}_{f}=f^{-1}(c) \cap \mathbb{B}_{\varepsilon}(0)$, definimos o número de Milnor de $V, \mu(V)$, como sendo o número de esferas do buquê de esferas $\mathbb{S}^{n}$.

\subsection{Variedades algébricas e espaços analíticos complexos}

Nesta seção trabalharemos com o caso complexo.

\section{Variedades algébricas}

O $n$-espaço afim sobre $\mathbb{C}$, denotado por $\mathbb{A}^{n}$ é o conjunto de todas as $n$-uplas de elementos de $\mathbb{C}$. Um ponto $p \in \mathbb{A}^{n}$ é dado por $p=\left(a_{1}, \ldots, a_{n}\right)$, com $a_{i} \in \mathbb{C}$, e $a_{i}$ a $i$-ésima coordenada de $p$.

Seja $\mathbb{C}\left[x_{1}, \cdots, x_{n}\right]$ o anel de polinômios em $n$ variáveis sobre $\mathbb{C}$. Podemos interpretar os elementos de $\mathbb{C}\left[x_{1}, \cdots, x_{n}\right]$ como funções do espaço afim $\mathbb{A}^{n}$ sobre $\mathbb{C}$, com $f(p)=$ $f\left(a_{1}, \ldots, a_{n}\right)$, onde $f \in \mathbb{C}\left[x_{1}, \cdots, x_{n}\right]$ e $p \in \mathbb{A}^{n}$. Desta forma, faz sentido falar no conjunto de zeros de $f$, que denotaremos por $Z(f)=\left\{p \in \mathbb{A}^{n} ; f(p)=0\right\}$. De uma forma mais geral, se $T$ for um subconjunto de $\mathbb{C}\left[x_{1}, \cdots, x_{n}\right]$, podemos definir o conjunto de zeros de $T$ como sendo o conjunto de zeros comuns a todos os elementos de $T$. Denotaremos esse 
conjunto da seguinte forma,

$$
Z(T)=\left\{p \in \mathbb{A}^{n} ; f(p)=0 \forall f \in T\right\}
$$

Claramente se $I$ for o ideal de $\mathbb{C}\left[x_{1}, \cdots, x_{n}\right]$ gerado por $T$ então $Z(T)=Z(I)$. Mais ainda, como $\mathbb{C}\left[x_{1}, \cdots, x_{n}\right]$ é um anel Noetheriano, qualquer ideal $I$ tem um número finito de geradores $f_{1}, \ldots, f_{r}$, logo $Z(T)$ pode se expressar como o conjunto de zeros comuns de um número finito de polinômios $f_{1}, \ldots, f_{r}$.

Definição 1.5.1. Um subconjunto $Y$ de $\mathbb{A}^{n}$ é chamado conjunto algébrico se existe um subconjunto $T \subset \mathbb{C}\left[x_{1}, \cdots, x_{n}\right]$ tal que $Y=Z(T)$.

Proposição 1.5.2. A união de dois conjuntos algébricos é um conjunto algébrico. A intersecção de uma família arbitrária de conjuntos algébricos também é um conjunto algébrico.

Para a demonstração deste resultado e referência básica para o assunto ver [12].

Definição 1.5.3. Os conjuntos algébricos são os conjuntos fechados de uma topologia de $\mathbb{A}^{n}$, denominada topologia de Zariski.

Definição 1.5.4. Dizemos que $Y \subset X$, um subconjunto não vazio $Y$ contido em $X$ é irredutível se o mesmo não pode se expressar como uma união $Y=Y_{1} \cup Y_{2}$, onde $Y_{1}, Y_{2}$ são fechados em $Y$.

Definição 1.5.5. Uma variedade algébrica afim é um subconjunto irredutível de $\mathbb{A}^{n}$ (com a topologia induzida).

\section{Espaços analíticos complexos}

Analogamente ao caso de polinômios, podemos também estudar o conjunto de zeros de uma ou mais funções analíticas. Estes são os chamados espaços analíticos.

Primeiramente consideremos a seguinte definição:

Definição 1.5.6. Consideremos o conjunto de pares $\left(V_{\alpha}, U_{\alpha}\right)$, onde $U_{\alpha}$ é uma vizinhança aberta da origem em $\mathbb{C}^{n}$ e $V_{\alpha}$ subconjuntos de $U_{\alpha}$. Dois tais pares $\left(V_{1}, U_{1}\right)$ e $\left(V_{2}, U_{2}\right)$ são 
equivalentes se existe uma vizinhança $W \subset U_{1} \cap U_{2}$ da origem tal que $V_{1} \cap W=V_{2} \cap W$. Uma classe de equivalência destes pares é chamada germe na origem em $\mathbb{C}^{n}$.

Se $f \in \mathcal{O}_{n}$, a classe de equivalência do conjunto $\{x: f(x)=0\}$, onde $f$ é um representante do germe $f$, é denotada por $\mathcal{V}(f)$; se $f_{1}$ e $f_{2}$ são dois representantes de um mesmo germe, então os conjuntos $\mathcal{V}\left(f_{1}\right)$ e $\mathcal{V}\left(f_{2}\right)$ são iguais.

Definição 1.5.7. Um germe de espaço analítico $(V, 0)$ em torno da origem é o germe do subconjunto

$$
V=\mathcal{V}\left(f_{1}\right) \cap \ldots \cap \mathcal{V}\left(f_{r}\right)=\mathcal{V}\left(f_{1}, \ldots, f_{r}\right),
$$

$\operatorname{para} f_{1}, \ldots, f_{r} \in \mathcal{O}_{n}$.

Definimos o ideal de um germe de espaço analítico $V$ por

$$
\mathcal{I}(V)=\left\{f \in \mathcal{O}_{n}: V \subset f^{-1}(0)\right\}
$$

Dizemos que um germe de espaço analítico $V$ é irredutível quando para quaisquer germes $V_{1}$ e $V_{2}$ tais que $V=V_{1} \cup V_{2}$ então $V=V_{1}$ ou $V=V_{2}$, neste caso dizemos que $V$ é uma variedade analítica.

O nosso objetivo é estudar a natureza de tais espaços analíticos na vizinhança de algum ponto fixado em $\mathbb{C}^{n}$, o qual sem perda de generalidade consideraremos a origem. Para isso precisamos definir formalmente o conceito de germe de um espaço analítico complexo (veja [10]).

Proposição 1.5.8. Seja $V$ um germe de espaço analítico, então existem um inteiro positivo $p$ e $V_{1}, \ldots, V_{p}$ variedades irredutíveis, com $V_{i}$ não contida em $V_{j}$, para todo $i \neq j$, tais que $V=V_{1} \cup \ldots \cup V_{p}$. Essas variedades são unicamente determinadas, a menos da ordem, e são chamadas de componentes irredutíveis de $V$.

Definição 1.5.9. Dizemos que um germe de espaço analítico $V$ é equidimensional quando todas as suas componentes irredutíveis tem a mesma dimensão.

Chamamos de germe de espaço analítico em $x$, um germe de conjunto $V$ em $x$ tal que, para alguma vizinhança $U$ de $x$, o germe $V \cap U$ pode ser descrito por $\mathcal{V}\left(f_{1}, \ldots, f_{r}\right)$, para alguns $f_{1}, \ldots, f_{r} \in \mathcal{O}_{n}$. 
Definição 1.5.10. Dizemos que um germe $V=\mathcal{V}\left(f_{1}, \ldots, f_{r}\right)$ é reduzido se a $\mathbb{C}$-álgebra $\frac{\mathcal{O}_{n}}{\left\langle f_{1}, \ldots, f_{r}\right\rangle}$ não possui elementos nilpotentes.

Dizemos que um ponto $z$ de um germe de espaço analítico $V$ é um ponto regular ou suave se para alguma vizinhança $U$ de $z$, o germe $U \cap V$ pode ser descrito como o conjunto dos zeros de um número finito de germes de funções analíticas que possuem $z$ como ponto regular. Um ponto de $V$ não regular é chamado de ponto singular de $V$.

Teorema 1.5.11 (Hilbert's Nullstellensatz - versão local,[9]). Seja $I$ um ideal de $\mathcal{O}_{n}$. Então $\mathcal{I}(\mathcal{V}(I))=\operatorname{Rad}(I)$, onde $\operatorname{Rad}(I)$ é o ideal radical de $I$, ou seja, o ideal $\left\{f \in \mathcal{O}_{n}\right.$ : $\exists n \in \mathbb{N}$ tal que $\left.f^{n} \in I\right\}$. 



\section{Capítulo}

\section{O índice de Poincaré-Hopf para campos}

de vetores em $\mathbb{R}^{2}$

Neste capítulo, como uma motivação para o estudo deste invariante no caso geral, apresentamos a definição do indice de Poincaré-Hopf de uma singularidade isolada $p$ de um campo de vetores $v$ definido em $\mathbb{R}^{2}$.

Antes de começarmos, façamos a seguinte observação: toda vez que nos referirmos a $\mathbb{R}^{2}$, estaremos considerando-o como o espaço topológico munido com a topologia usual.

São inúmeros os fenômenos físicos e naturais que podem ser estudados através de campos de vetores. Os vetores podem representar, por exemplo, campos de velocidade, como a velocidade dos ventos e a velocidade das correntes oceânicas. As Figuras 2.1 e 2.2 ilustram vetores velocidade do ar e indicam a rapidez, a direção e o sentido em pontos $10 \mathrm{~m}$ acima da superfície na área da baía de São Francisco. A Figura 2.1 representa os registros feitos no dia 11 de junho de 2002 as 12 horas, já a Figura 2.2 mostra as ocorrências registradas no dia 30 de junho de 2002 as 16 horas. Observemos que as duas figuras apresentam aspectos bastante diferentes. 


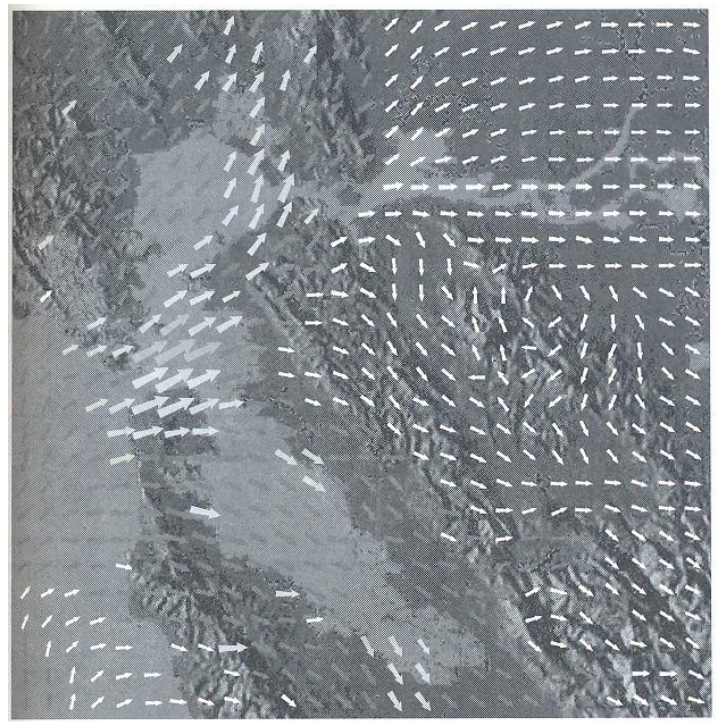

Figura 2.1: Registros do dia $11 / 06 / 2002$

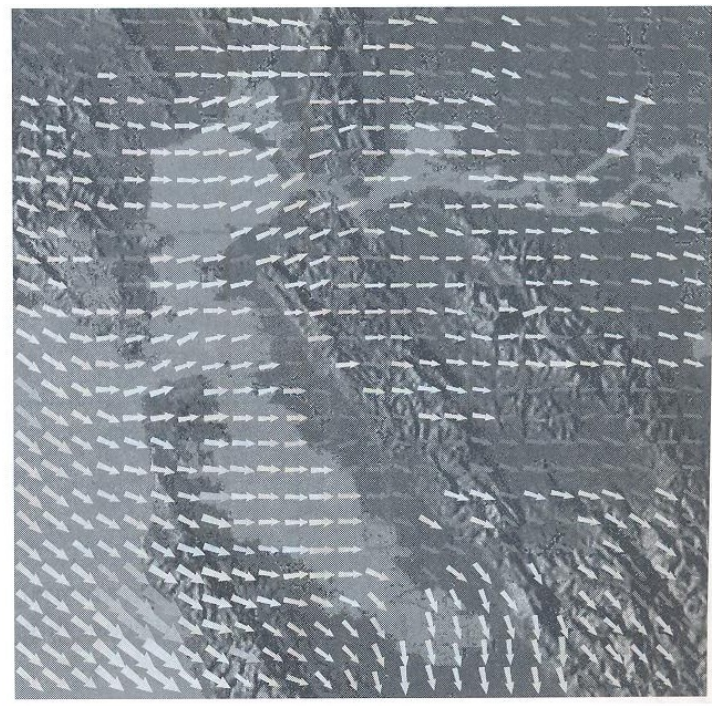

Figura 2.2: Registros do dia $30 / 06 / 2002$

Campos vetoriais também são utilizados para representar a direção de ondas. Como ilustra a Figura 2.3, gerada pelo Instituto Nacional de Pesquisas Espaciais (INPE).

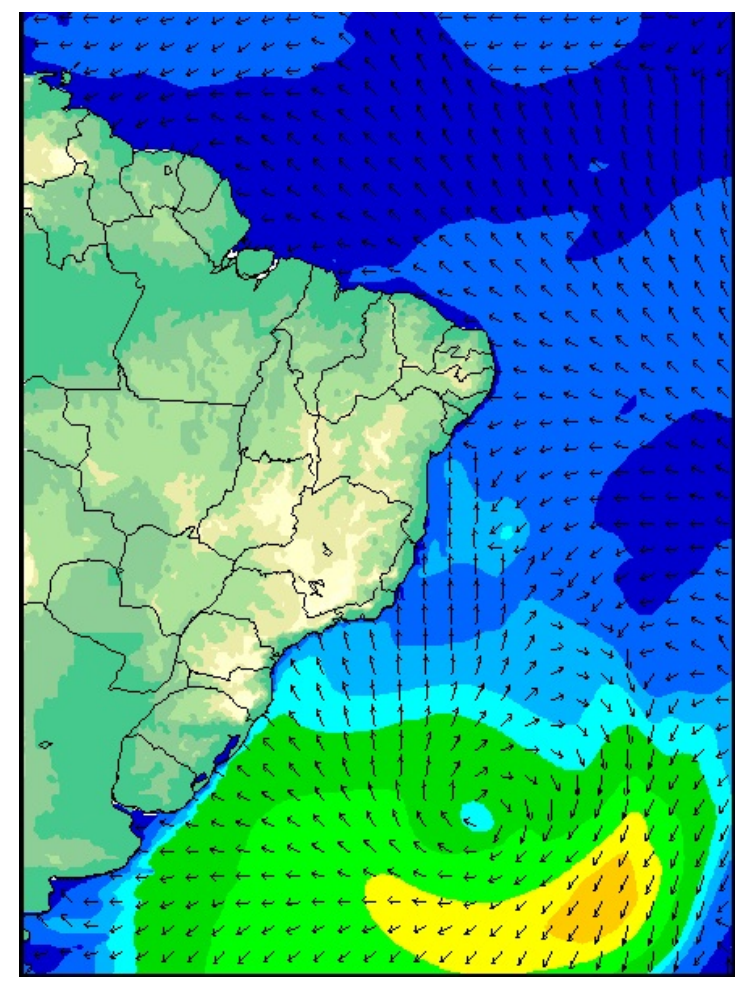

Figura 2.3: Direção da onda

Vejamos então, a definição do importante conceito de campos de vetores. 
Definição 2.0.12. Dado $U$ um subconjunto aberto de $\mathbb{R}^{2}$, um campo de vetores em $U$ é uma aplicação $v: U \rightarrow \mathbb{R}^{2}$ que a cada ponto $(x, y) \in U$ associa o elemento

$$
v(x, y)=\left(v_{1}(x, y), v_{2}(x, y)\right)
$$

O campo $v: U \rightarrow \mathbb{R}^{2}$ é dito contínuo, diferenciável ou de classe $C^{k}$ em $p \in U$ se suas funções coordenadas

$$
v_{i}: U \rightarrow \mathbb{R}, \quad i=1,2
$$

são contínuas, diferenciáveis ou de classe $C^{k}$ em $p \in U$, respectivamente.

O nome se justifica se expressarmos graficamente $v$ do seguinte modo: em cada ponto $(x, y) \in U$ desenhamos um vetor de magnitude e direção de $v(x, y)$ com a origem em $(x, y)$. Como podemos ver na figura do exemplo abaixo.

Exemplo 2.0.13. Consideremos a função diferenciável $f: \mathbb{R}^{2} \rightarrow \mathbb{R}$ dada por $f(x, y)=\frac{y^{2}}{2}-\frac{x^{3}}{3}+x$. A aplicação $v: \mathbb{R}^{2} \rightarrow \mathbb{R}^{2}$, definida por

$$
v(x, y)=\left(\frac{\partial f}{\partial y}(x, y),-\frac{\partial f}{\partial x}(x, y)\right)=\left(y, x^{2}-1\right)
$$

pode ser vista como um campo de vetores em $\mathbb{R}^{2}$.

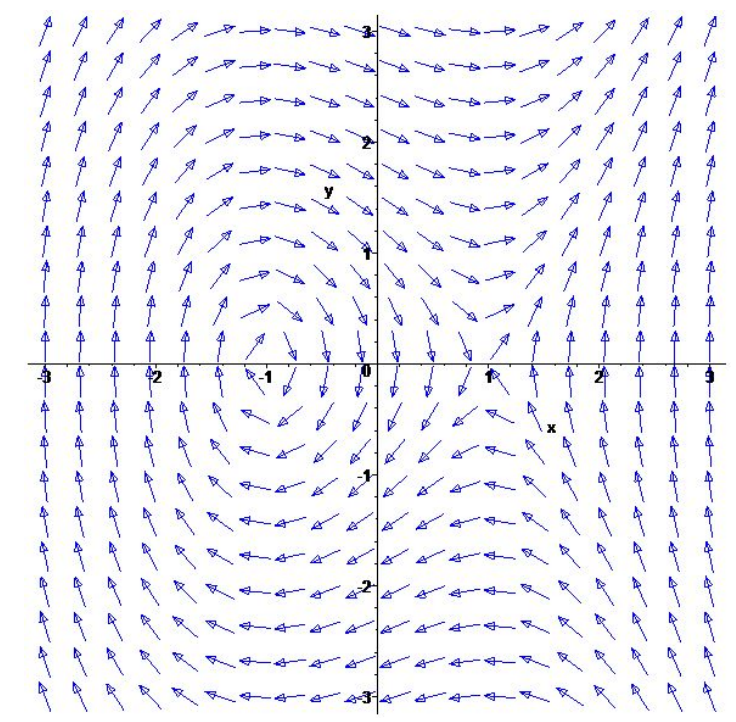

Figura 2.4: Campo de vetores $v$ em $\mathbb{R}^{2}$ 
Observemos, que neste exemplo, o campo de vetores $v$ se anula nos pontos $(-1,0)$ e $(1,0)$.

Definição 2.0.14. Sejam $U$ um aberto de $\mathbb{R}^{2}$ e $v$ um campo de vetores em $U$, dizemos que um ponto $p \in U$ é uma singularidade de $v$ quando $v(p)=(0,0)$.

Assim, os pontos $(-1,0)$ e $(1,0)$ são singularidades do campo vetorial apresentado no exemplo anterior. Além disso, notemos que existem vizinhanças $V_{1}, V_{2}$ de $(-1,0)$ e $(1,0)$, respectivamente, em $\mathbb{R}^{2}$ tais que $v(-1,0)=v(1,0)=(0,0)$ e para todos os outros pontos $(x, y)$ pertencentes a $V_{1} \cup V_{2}$, temos $v(x, y) \neq(0,0)$. As singularidades de um campo vetorial com essa propriedade recebem um nome especial.

Definição 2.0.15. Sejam $U$ um aberto de $\mathbb{R}^{2}$ e $v$ um campo de vetores em $U$. Se $p \in U$ é uma singularidade de $v$ e existe uma vizinhança $V_{p}$ de $p$ em $U$ tal que $p$ é a única singularidade de $v$ em $V_{p}$, então dizemos que $p$ é uma singularidade isolada de $v$.

Exemplo 2.0.16. Consideremos o campo de vetores $v: \mathbb{R}^{2} \rightarrow \mathbb{R}^{2}$ dado por

$$
v(x, y)=(\cos (x) \operatorname{sen}(y), \operatorname{sen}(x) \cos (y))
$$

que é representado na Figura 2.5. Dados $k_{1}, k_{2} \in \mathbb{Z}$, temos que os pontos da forma

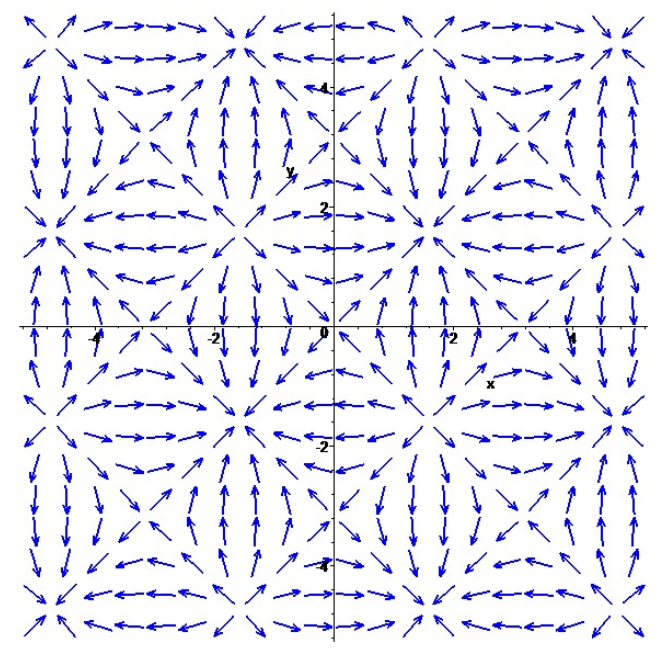

Figura 2.5: Campo de vetores $v$ em $\mathbb{R}^{2}$

$\left(k_{1} \frac{\pi}{2}, k_{2} \frac{\pi}{2}\right)$ e $\left(k_{1} \pi, k_{2} \pi\right)$ são todos singularidades isoladas de $v$. 
Para definirmos o índice de Poincaré-Hopf precisaremos da noção de orientação sobre circunferências. Se orientarmos o espaço $\mathbb{R}^{2}$ positivamente pela base canônica, geometricamente, fica natural definirmos uma orientação sobre uma circunferência de $\mathbb{R}^{2}$, como na definição a seguir.

Definição 2.0.17. Seja $\mathbb{S}_{\varepsilon}$ uma circunferência de raio $\varepsilon$ em $\mathbb{R}^{2}$, definiremos o sentido positivo de $\mathbb{S}_{\varepsilon}$ como sendo o sentido anti-horário de $\mathbb{S}_{\varepsilon}$. O sentido negativo será o dado pelo sentido horário de $\mathbb{S}_{\varepsilon}$.
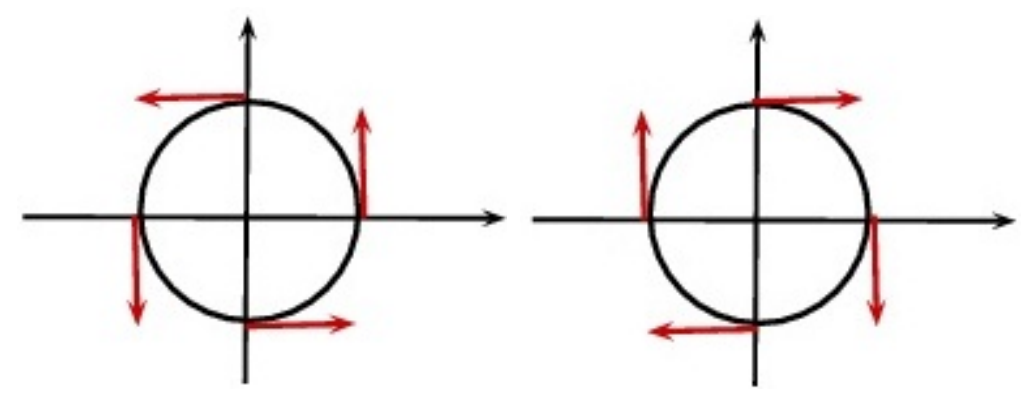

Figura 2.6: Sentido positivo e negativo, respectivamente, em $\mathbb{S}^{1}$.

\subsection{O índice de Poincaré-Hopf}

Dados $U$ um subconjunto aberto de $\mathbb{R}^{2}$ e $v: U \rightarrow \mathbb{R}^{2}$ um campo de vetores com uma singularidade isolada $p$. Nesta seção, definiremos o índice de Poincaré-Hopf de $v$ em $p$. Para definirmos este conceito utilizaremos a definição de orientação sobre uma circunferência $\mathbb{S}_{\varepsilon}$ de $\mathbb{R}^{2}$, que apresentamos na seção anterior, e também precisaremos de uma aplicação bastante especial, chamada de aplicação de Gauss. Comecemos então, apresentando a aplicação de Gauss.

Definição 2.1.1. Consideremos $U$ um subconjunto aberto de $\mathbb{R}^{2}$ e $v: U \rightarrow \mathbb{R}^{2}$ um campo de vetores contínuo com uma singularidade isolada $p$. Seja $\mathbb{S}_{\varepsilon} \subset U$ uma pequena esfera centrada em $p$ de raio $\varepsilon$. Então, a aplicação

$$
\gamma: \mathbb{S}_{\varepsilon} \rightarrow \mathbb{S}^{1}
$$

definida por $\gamma(x)=v(x) /\|v(x)\|$, é chamada de aplicação de Gauss. 
A aplicação de Gauss $\gamma$ transforma cada vetor dado pelo campo $v$ em um vetor unitário de $\mathbb{R}^{2}$, como mostra a Figura 2.7.

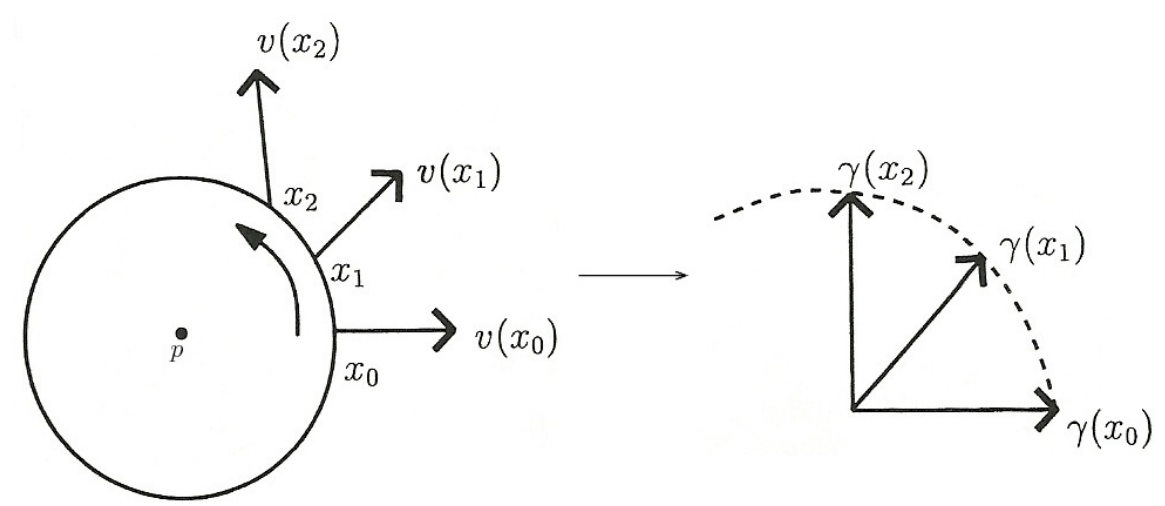

Figura 2.7: Aplicação de Gauss

Observação 2.1.2. Nesta seção, a orientação das circunferências consideradas será sempre a induzida pela orientação de $\mathbb{R}^{2}$ (ver Definição 2.0.17), que por sua vez será sempre orientado positivamente pela base canônica.

Definição 2.1.3. Dados $U$ um subconjunto aberto de $\mathbb{R}^{2}$ e $v: U \rightarrow \mathbb{R}^{2}$ um campo de vetores com uma singularidade isolada $p$. Se a aplicação de Gauss $\gamma$ percorre a circunferência $\mathbb{S}^{1}$ no sentido anti-horário quando o campo $v$ percorre a circunferência $\mathbb{S}_{\varepsilon}$ no sentido anti-horário, diremos que $\gamma$ percorre $\mathbb{S}^{1}$ positivamente. Caso contrário, diremos que $\gamma$ percorre $\mathbb{S}^{1}$ negativamente.

Utilizando a Definição 2.1.3 podemos definir o índice de Poincaré-Hopf de uma singularidade isolada de um campo de vetores da seguinte forma.

Definição 2.1.4. Considere um aberto $U$ de $\mathbb{R}^{2}$ e $v$ um campo de vetores contínuo em $U$ com uma singularidade isolada $p$. Seja $\mathbb{S}_{\varepsilon}$ uma pequena circunferência centrada em $p$ de raio $\varepsilon$. O índice de Poincaré-Hopf de $v$ em $p$ é por definição o número de voltas positivas menos o número de voltas negativas que a aplicação $\gamma$ faz em torno de $\mathbb{S}^{1}$ quando o campo $v$ percorre toda a circunferência $\mathbb{S}_{\varepsilon}$.

Denotaremos o índice de Poincaré-Hopf do campo $v$ em $p$ por $\operatorname{Ind}_{P H}(v, p)$.

A definição acima tem um caráter bastante geométrico. Assim, os exemplos que apresentaremos a seguir também assumirão este caráter. 
Exemplo 2.1.5. A Figura 2.8 traz representações geométricas de três campos de vetores contínuos, cada um com uma singularidade isolada no ponto $a$.

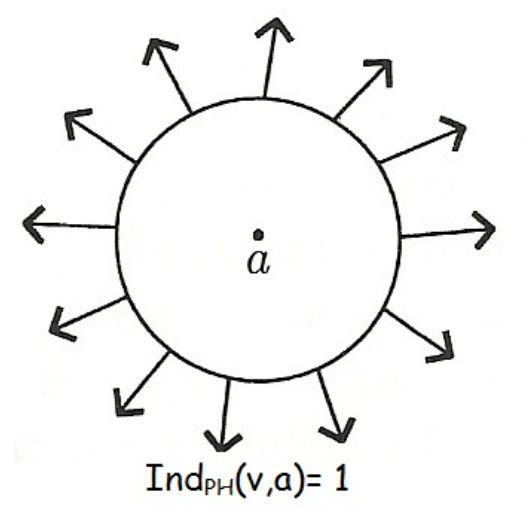

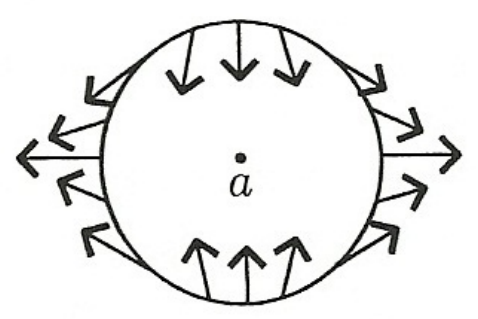

$\operatorname{Ind}_{P H}(v, a)=-1$

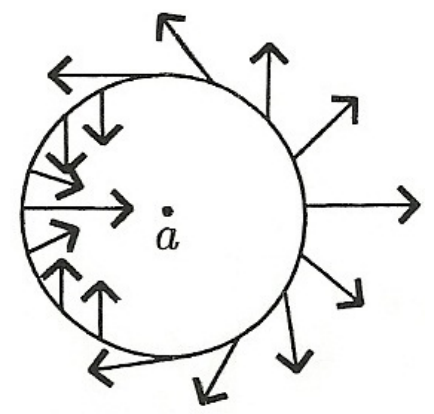

$\operatorname{Ind}_{P H}(v, a)=2$

Figura 2.8: Campos de vetores em dimensão 2

Exemplo 2.1.6. Consideremos o campo de vetores $v: \mathbb{R}^{2} \rightarrow \mathbb{R}^{2}$ dado por $v(x, y)=$ $(\cos (x) \operatorname{sen}(y), \operatorname{sen}(x) \cos (y))$. Como vimos no Exemplo 2.0.16, dados $k_{1}, k_{2} \in \mathbb{Z}$, todos os pontos da forma $\left(k_{1} \frac{\pi}{2}, k_{2} \frac{\pi}{2}\right)$ e $\left(k_{1} \pi, k_{2} \pi\right)$ são singularidades isoladas de $v$, como mostra a Figura 2.9. Os pontos da forma $\left(k_{1} \frac{\pi}{2}, k_{2} \frac{\pi}{2}\right)$ tem índice de Poincaré-Hopf é igual a 1, já

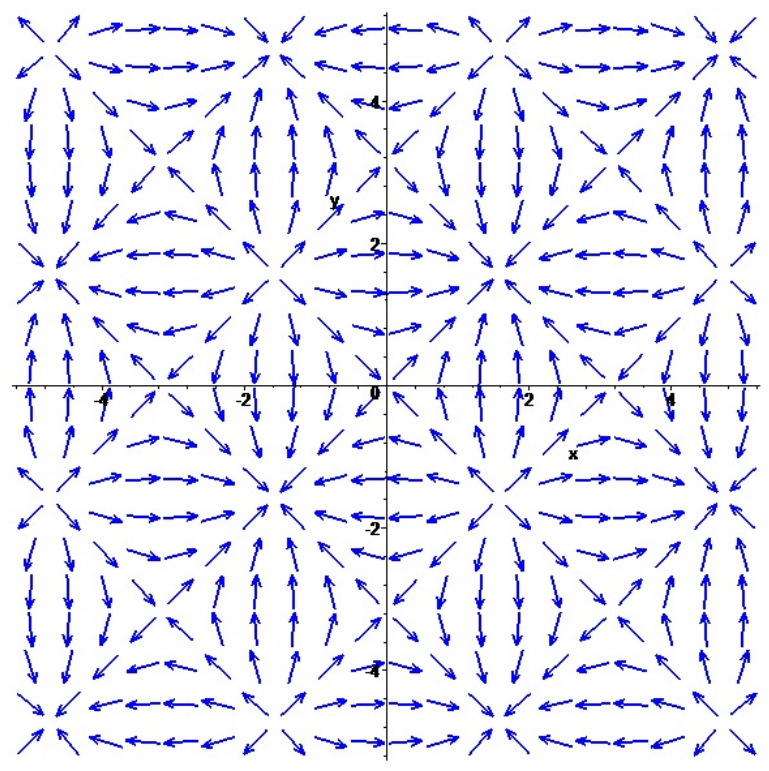

Figura 2.9: Campo de vetores $v$ em $\mathbb{R}^{2}$

para os pontos da forma $\left(k_{1} \pi, k_{2} \pi\right)$ o índice é igual a -1 .

Em nosso próximo exemplo, estudaremos um campo de vetores no $\mathbb{R}^{3}$ restrito a esfera $\mathbb{S}^{2}$, motivando assim o estudo de campos de vetores sobre superfícies e variedades. 
Exemplo 2.1.7. Consideremos o campo de vetores em $\mathbb{R}^{3}$

$$
v: \mathbb{R}^{3} \rightarrow \mathbb{R}^{3}
$$

cuja restrição a esfera unitária de $\mathbb{R}^{3}$ é a dada pela Figura 2.10 .

Observemos que sobre a esfera $\mathbb{S}^{2}$ este campo se anula apenas nos pontos $(0,0,-1)$ e $(0,0,1)$, como ilustra a Figura 2.10 .

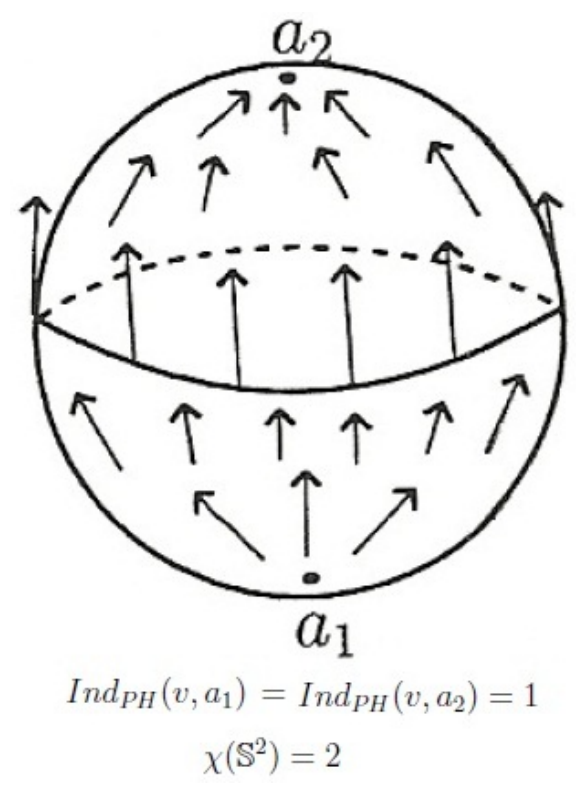

Figura 2.10: Aplicação $v$ sobre $\mathbb{S}^{2}$

Podemos ver esta aplicação como um modelo simplificado do campo magnético terrestre (veja a Figura 2.11).

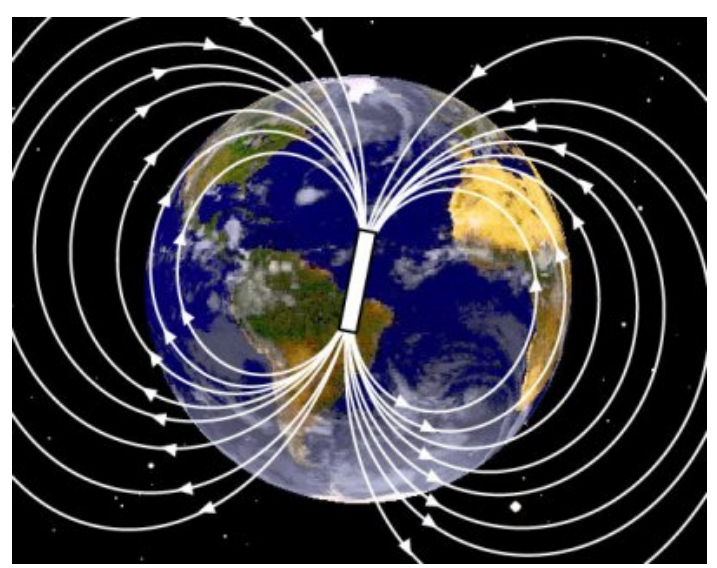

Figura 2.11: Campo magnético terrestre 
Devido ao comportamento da aplicação $v$ em torno dos pontos $(0,0,-1)$ e $(0,0,1)$, podemos pensar que o comportamento do campo em torno de suas singularidades, é similar ao descrito na Figura 2.12 .
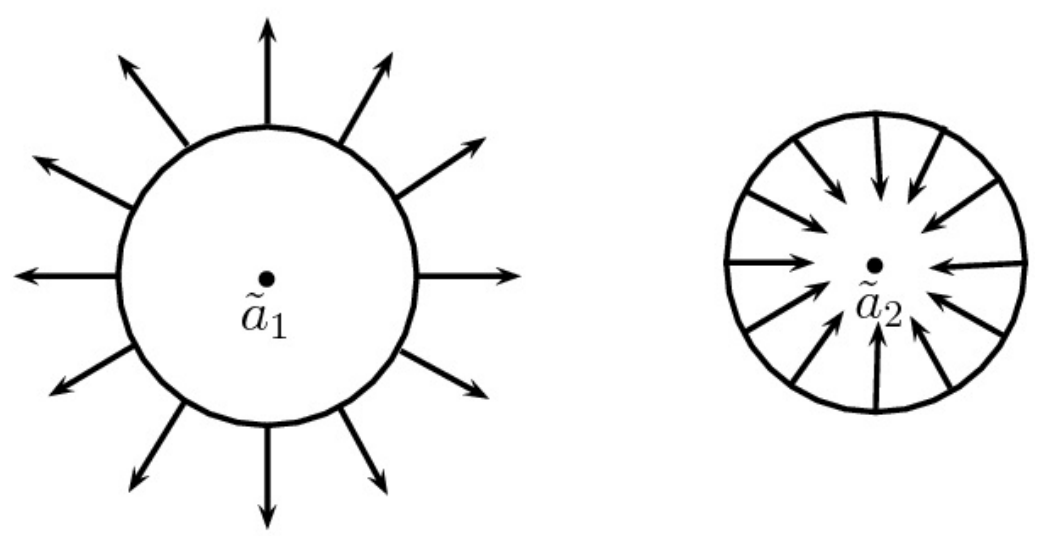

Figura 2.12: Campo em torno de suas singularidades

então, parece natural que os indices das singularidades deste campo restrito a esfera sejam ambos iguais $\mathrm{a}+1$.

Motivados por este exemplo, no decorrer deste trabalho, formalizaremos esta ideia para definirmos o índice de Poincaré-Hopf para singularidades de campos de vetores definidos sobre variedades diferenciáveis. Além disso, após um estudo deste índice generalizaremos este objeto para variedades singulares. 



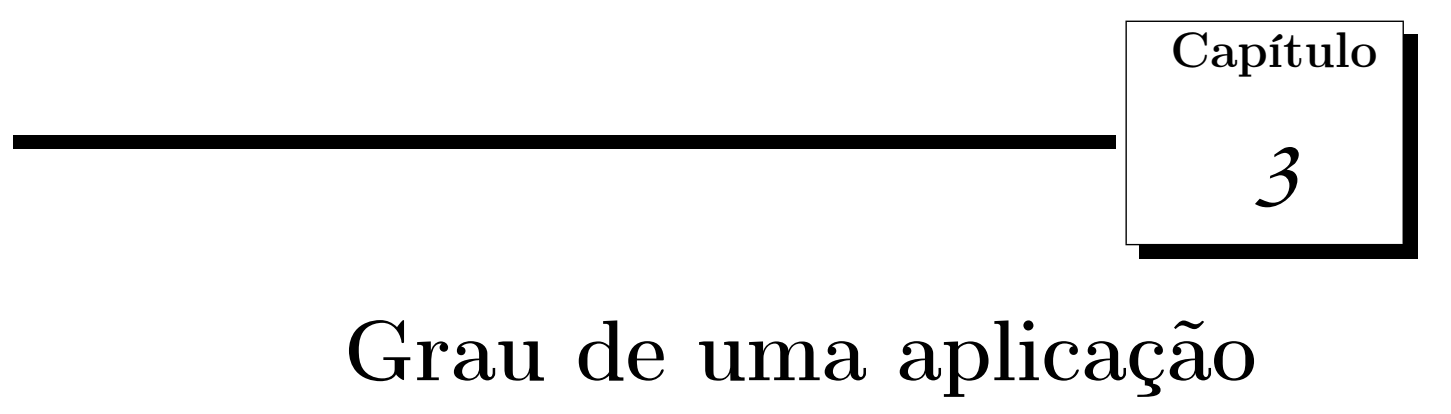

Como dito anteriormente, no capítulo 4 definiremos o índice de Poincaré-Hopf para singularidades isoladas de campos de vetores contínuos sobre uma variedade diferenciável M. Tal conceito estará inteiramente relacionado com a noção de grau de uma aplicação diferenciável própria $f: M \rightarrow N$, onde $M, N$ são variedades diferenciáveis orientadas de mesma dimensão $m$.

Na verdade, o índice de Poincaré-Hopf de uma singularidade isolada será definido como o grau de uma aplicação especial. Assim, neste capítulo faremos um estudo do conceito de grau de uma aplicação diferenciável própria.

Começaremos então, com a definição de aplicações próprias.

Definição 3.0.8. Sejam $X$ e $Y$ espaços topológicos localmente compactos. Dizemos que uma aplicação contínua $f: X \rightarrow Y$ é própria quando dado um subconjunto compacto $K$ de $Y$ a sua imagem inversa $f^{-1}(K)$ for um subconjunto compacto de $X$.

A seguir, apresentaremos alguns exemplos e contra-exemplos de aplicações próprias.

Exemplo 3.0.9. A função $f: \mathbb{R} \rightarrow \mathbb{R}$ dada por $f(x)=x^{2}$ é própria. De fato, dado um subconjunto compacto $K \subset \mathbb{R}$, sabemos que $K$ é fechado e limitado. Agora, como a função $f$ é contínua segue que $f^{-1}(K)$ é um subconjunto fechado de $\mathbb{R}$, além disso, $f^{-1}(K)$ também é um subconjunto limitado de $\mathbb{R}$, pois basta observarmos que $f^{-1}([0, R])=$ $(-\sqrt{R}, \sqrt{R})$ para todo $R>0$ pertencente a $\mathbb{R}$. Portanto, a função $f$ é própria. 
Por outro lado, a função $g:(0,1) \rightarrow[0,1]$ definida por $g(x)=x^{2}$, não é própria, uma vez que $g^{-1}([0,1])=(0,1)$ não é um compacto.

Exemplo 3.0.10. A função $f: \mathbb{R} \rightarrow \mathbb{R}$ definida por $f(x)=\operatorname{sen}(x)$ para todo $x \in \mathbb{R}$ não é própria. De fato, tomemos o subconjunto compacto $K=\{0\}$ de $\mathbb{R}$, temos que $f^{-1}(K)=\{k \pi, \quad k \in \mathbb{Z}\}$ não é um subconjunto compacto de $\mathbb{R}$, pois este conjunto não é limitado.

Exemplo 3.0.11. Consideremos a função

$$
\begin{aligned}
& f: \mathbb{R}^{2} \rightarrow \mathbb{R} \\
& (x, y) \mapsto x^{2}+y^{2} .
\end{aligned}
$$

Fazendo uma análise semelhante a que fizemos no Exemplo 3.0.9, concluímos que a aplicação $f$ é própria, uma vez que $f^{-1}([0, R])=\bar{B}_{\sqrt{R}}(0)$, onde $\bar{B}_{\sqrt{R}}(0)$ denota a bola fechada de centro 0 e raio $\sqrt{R}$ de $\mathbb{R}^{2}$.

Exemplo 3.0.12. A função $f: \mathbb{R}^{2} \rightarrow \mathbb{R}$, dada por $f(x, y)=x$, não é própria, uma vez que para todo ponto $a \in \mathbb{R}$ a imagem inversa $f^{-1}(a)$ é uma reta em $\mathbb{R}^{2}$ passando por $(a, 0)$.

A seguir, apresentaremos uma proposição, que nos fornece uma importante classe de exemplos de aplicações próprias.

Proposição 3.0.13. Se $X$ é um espaço topológico compacto e $Y$ é um espaço topológico localmente compacto Hausdorff, toda aplicação contínua $f: X \rightarrow Y$ é própria.

Demonstração: Dado um compacto $K \subset Y$, como $Y$ é Hausdorff segue que $K$ é um fechado em $Y, \log o f^{-1}(K)$ é um fechado em $X$. Mas $X$ é compacto, donde temos que $f^{-1}(K)$ é um compacto em $X$.

Observação 3.0.14. Sejam $f: X \rightarrow Y$ uma aplicação própria, $B \subset Y$ um subconjunto aberto em $Y$ e $A=f^{-1}(B)$, então a aplicação $\tilde{f}: A \rightarrow B$ definida por $\tilde{f}(x)=f(x)$, para todo $x \in A$, é também própria. Notemos, que $A$ e $B$ são localmente compactos, por serem subconjuntos abertos de espaços localmente compactos. 


\subsection{Grau de uma aplicação}

Sejam $M, N$ variedades diferenciáveis $n$-dimensionais, orientadas e $f: M \rightarrow N$ uma aplicação diferenciável própria. Como a variedade $N$ é um espaço Hausdorff, dado um ponto $p \in N$ temos que $K=\{p\}$ é um subconjunto compacto de $N$, e portanto $f^{-1}(p)$ é um subconjunto compacto de $M$.

No caso em que $p$ é um valor regular de $f$, sua imagem inversa $f^{-1}(p)$ é uma subvariedade 0-dimensional de $M$ (ver Teorema 1.1.14), o que significa que $f^{-1}(p)$ é uma quantidade finita de pontos de $M$, dessa maneira, a seguinte definição faz sentido.

Definição 3.1.1. Sejam $M, N$ variedades diferenciáveis $n$-dimensionais, orientadas e $f: M \rightarrow N$ uma aplicação diferenciável própria. Dado $p \in N$ um valor regular de $f$, o grau de $f$ relativamente ao valor regular $p$ é o número algébrico de pontos em $f^{-1}(p)$, isto é, o número de pontos positivos menos o número de pontos negativos em $f^{-1}(p)$.

A noção de pontos positivos e pontos negativos pode ser vista na Definição 1.1.28.

Para indicar o grau de $f$ relativamente a um valor regular $p$, usaremos a notação $g r_{p}(f)$.

Exemplo 3.1.2. Consideremos a função $f: \mathbb{R} \rightarrow \mathbb{R}$ definida por $f(x)=x^{2}$. Para todo $h \in \mathbb{R}$, a derivada de $f$ em $h$ é a aplicação linear $d f_{h}: \mathbb{R} \rightarrow \mathbb{R}$ dada por $d f_{h}(x)=2 h x$. Assim, temos três casos a considerar:

1) Se $h>0$, então $h$ é um valor regular de $f$ e $f^{-1}(h)=\{-\sqrt{h}, \sqrt{h}\}$, além disso

$$
d f_{-\sqrt{h}}(x)=-2 \sqrt{h} x
$$

é uma função sobrejetora que inverte a orientação. Por outro lado, $d f_{\sqrt{h}}(x)=2 \sqrt{h} x$ é sobrejetora e preserva a orientação, $\log 0 g r_{h}(f)=0$;

2) Se $h=0$, então $d f_{h}$ é a função identicamente nula, assim $h=0$ não é valor regular de $f$;

3) Se $h<0$, então $f^{-1}(h)=\varnothing$ e consequentemente $g r_{h}(f)=0$. 
Analisando estes três casos podemos concluir que $g r_{h}(f)=0$.

Exemplo 3.1.3. Seja $\mathbb{S}^{1}$ a circunferência unitária do plano. Para cada inteiro $n \in \mathbb{Z}$, consideremos a aplicação

$$
f_{n}: \mathbb{S}^{1} \rightarrow \mathbb{S}^{1}
$$

definida por $f_{n}(\cos (\theta), \operatorname{sen}(\theta))=(\cos (n \theta), \operatorname{sen}(n \theta))$. Pensando cada ponto $z=(x, y)=$ $(\cos (\theta), \operatorname{sen}(\theta))$ em $\mathbb{S}^{1}$ como o número complexo de módulo $1, z=x+i y=\cos (\theta)+i \operatorname{sen}(\theta)$, temos $f_{n}(z)=z^{n}$.

Consideremos primeiro o caso $n \neq 0$. Para cada $p \in \mathbb{S}^{1}$, o subconjunto $f_{n}^{-1}(p)$ contém exatamente $|n|$ pontos, digamos

$$
f_{n}^{-1}(p)=\left\{q_{1}, \ldots, q_{|n|}\right\}
$$

Como a aplicação $h:(-\varepsilon, 2 \pi) \rightarrow \mathbb{S}^{1}$ definida por $h(\theta)=(\cos (\theta)$, $\operatorname{sen}(\theta))$ é um difeomorfismo local, dado $q_{i} \in f_{n}^{-1}(p)$, existe um intervalo aberto $\left(\theta_{1}, \theta_{2}\right)$, satisfazendo $\left|\theta_{1}-\theta_{2}\right|<2 \pi$ tal que a aplicação

$$
\begin{array}{ccc}
h:\left(\theta_{1}, \theta_{2}\right) & \rightarrow & \mathbb{S}^{1} \\
\theta & \mapsto & (\cos (\theta), \operatorname{sen}(\theta))
\end{array}
$$

é um difeomorfismo sobre sua imagem. Da mesma forma, a aplicação $k:(-n \varepsilon, 2 n \pi) \rightarrow \mathbb{S}^{1}$ definida por $k(\tau)=(\cos (\tau)$, $\operatorname{sen}(\tau))$ é um difeomorfismo local, então, considerando a função

$$
\begin{array}{rlc}
g_{n}:(-\varepsilon, 2 \pi) & \rightarrow & (-n \varepsilon, 2 n \pi) \\
\theta & \mapsto & n \theta
\end{array}
$$

e denotando por $\alpha$ e $\beta$ os pontos $h^{-1}\left(q_{i}\right)$ e $g_{n}(\alpha)$, respectivamente, obteremos um intervalo aberto $\left(\tau_{1}, \tau_{2}\right)$ satisfazendo $\left|\tau_{1}-\tau_{2}\right|<2 \pi$ tal que a aplicação

$$
\begin{array}{rlc}
k:\left(\tau_{1}, \tau_{2}\right) & \rightarrow & \mathbb{S}^{1} \\
\tau & \mapsto \quad(\cos (\tau), \sin (\tau))
\end{array}
$$


é um difeomorfismo sobre sua imagem, $\operatorname{com} \beta=g_{n}(\alpha) \in\left(\tau_{1}, \tau_{2}\right)$. Agora, observemos que para todo $q \in h\left(\theta_{1}, \theta_{2}\right)$, temos

$$
k \circ g_{n} \circ h^{-1}(q)=f_{n}(q)
$$

como ilustra o diagrama abaixo.

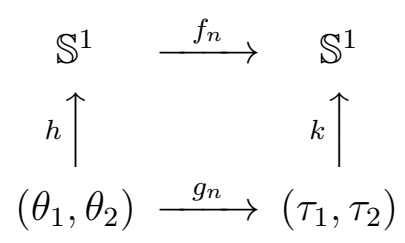

Mas,

$$
d\left(k \circ g_{n} \circ h^{-1}\right)_{q_{i}}: T_{q_{i}} \mathbb{S}^{1} \rightarrow T_{p} \mathbb{S}^{1}
$$

é um isomorfismo, logo a aplicação

$$
d\left(f_{n}\right)_{q_{i}}: T_{q_{i}} \mathbb{S}^{1} \rightarrow T_{p} \mathbb{S}^{1}
$$

também é um isomorfismo. Assim, cada $q_{i}$ é um ponto regular de $f_{n}$, e então $p$ é um valor regular de $f_{n}$. Agora, como $\left.h\right|_{\left(\theta_{1}, \theta_{2}\right)}$ e $\left.k\right|_{\left(\tau_{1}, \tau_{2}\right)}$ tem a mesma lei, o sinal do ponto $q_{i} \in \mathbb{S}^{1}$ relativamente a transformação $d\left(k \circ g_{n} \circ h^{-1}\right)_{q_{i}}$ é igual ao sinal de $\alpha$ relativamente a transformação $d\left(g_{n}\right)_{\alpha}$.

Logo, se $n<0$, todos os pontos de $f_{n}^{-1}(p)$ são negativos e se $n>0$, todos os pontos de $f_{n}{ }^{-1}(p)$ são positivos, portanto, $g r_{p}\left(f_{n}\right)=n$.

Quando $n=0$, temos $f_{0}(z)=(1,0)$ para todo $z \in \mathbb{S}^{1}$, então $d\left(f_{0}\right)_{v} \equiv 0$, para todo $v \in \mathbb{S}^{1}$. Dessa forma, os valores regulares de $f_{0}$ são os pontos $p \neq(1,0)$ e $g r_{p}\left(f_{0}\right)=0$.

Exemplo 3.1.4. Sejam $\mathbb{S}^{n} \subset \mathbb{R}^{n+1}$ a esfera unitária $n$-dimensional e a aplicação diferenciável $f: \mathbb{S}^{n} \rightarrow \mathbb{S}^{n}$ dada por

$$
f\left(x_{1}, \ldots, x_{n+1}\right)=\left(x_{1}, \ldots, x_{n},-x_{n+1}\right)
$$

em outras palavras, $f$ é a reflexão relativamente ao hiperplano $x_{n+1}=0$. 
Consideremos o ponto $p=(0, \ldots, 0,-1)$ em $\mathbb{S}^{n}$, então $f^{-1}(p)=q=(0, \ldots, 0,1)$. Os espaços vetoriais tangentes a $\mathbb{S}^{n}$ nos pontos $p$ e $q, T_{p} \mathbb{S}^{n}$ e $T_{q} \mathbb{S}^{n}$, são paralelos, como subespaços vetoriais do $\mathbb{R}^{n+1}$ ambos coincidem com o conjunto dos vetores $v \in \mathbb{R}^{n+1}$ tais que $v=\left(\alpha_{1}, \ldots, \alpha_{n}, 0\right)$.

No que diz respeito a orientação, diremos que uma base $\left\{e_{1}, \ldots, e_{n}\right\}$ de um espaço tangente $T_{u} \mathbb{S}^{n}$ é positiva se, completando-a com o vetor normal $\vec{v}=u-0$ que aponta para o exterior de $\mathbb{S}^{n}$, obtivermos uma base positiva $\left\{e_{1}, \ldots, e_{n}, v\right\}$ de $\mathbb{R}^{n+1}$.

Por exemplo, a base $\left\{e_{1}, \ldots, e_{n}\right\}$ formada pelos vetores

$$
e_{1}=(1,0, \ldots, 0,0), \ldots, e_{n}=(0, \ldots, 0,1,0)
$$

é positiva para o espaço tangente $T_{q} \mathbb{S}^{n}$, pois $\vec{v}=q-0=(0, \ldots, 0,1)$ determina a base positiva $\left\{e_{1}, \ldots, e_{n}, v\right\}$ em $\mathbb{R}^{n+1}$. Por outro lado, no ponto

$$
p=(0, \ldots, 0,-1)=f(q)
$$

a mesma base $\left\{e_{1}, \ldots, e_{n}\right\}$, agora considerada como base de $T_{p} \mathbb{S}^{n}$, é negativa, pois $\vec{w}=$ $p-0=(0, \ldots, 0,-1)$ determina a base negativa $\left\{e_{1}, \ldots, e_{n}, w\right\}$ para $\mathbb{R}^{n+1}$.

Agora, observemos que a transformação linear $d f_{q}: T_{q} \mathbb{S}^{n} \rightarrow T_{p} \mathbb{S}^{n}$ é dada por

$$
d f_{q}\left(h_{1}, \ldots, h_{n}, h_{n+1}\right)=\left(h_{1}, \ldots, h_{n},-h_{n+1}\right)
$$

$\log \mathrm{O}$

$$
d f_{q}\left(e_{1}\right)=e_{1}, \ldots, d f_{q}\left(e_{n}\right)=e_{n}
$$

Assim, $d f_{q}$ inverte a orientação e, por conseguinte, o ponto $q$ é negativo. Podemos então afirmar que $g r_{p}(f)=-1$.

Nesta seção, utilizaremos algumas aplicações do Teorema de Sard. Como, por exemplo, a Proposição 3.1 .5 nos garante que, sendo $f: M \rightarrow N$ uma aplicação própria de classe $C^{1}$, além do conjunto dos valores regulares de $f$ ser denso em $N$, esse conjunto é aberto em $N$. 
Proposição 3.1.5. Sejam $M, N$ variedades diferenciáveis $n$-dimensionais e $f: M \rightarrow N$ uma aplicação própria de classe $C^{1}$. Então, o subconjunto $R$ de $N$ formado por todos os valores regulares de $f$ é aberto em $N$.

Demonstração: Consideremos $R_{1}$ o subconjunto de $N$ definido abaixo

$$
R_{1}=\left\{p \in N: \quad p \text { é valor regular de } f \text { e } f^{-1}(p) \neq \varnothing\right\}
$$

Então, dado $p \in R_{1}$, a imagem inversa $f^{-1}(p)$ é uma subvariedade compacta 0 -dimensional de $M$, consequentemente é uma quantidade finita de pontos, digamos

$$
f^{-1}(p)=\left\{p_{1}, \ldots, p_{k}\right\} \subset M
$$

Além disso, para cada $p_{i} \in f^{-1}(p)$, a aplicação $d f_{p_{i}}: T_{p_{i}} M \rightarrow T_{p} N$ é um isomorfismo. Logo, pelo teorema da função inversa, existem vizinhanças abertas $U_{p_{i}}$ (as quais podemos tomar duas a duas disjuntas), de $p_{i}$ em $M$ e $V_{p, p_{i}}$ de $p$ em $N$ tais que

$$
f: U_{p_{i}} \rightarrow V_{p, p_{i}}
$$

é um difeomorfismo. Consideremos

$$
V=\bigcap_{i=1}^{k} V_{p, p_{i}} \subset V_{p, p_{i}}, \quad i=1, \ldots, k
$$

Como $p \in V$ e a aplicação

$$
\begin{aligned}
& \text { det }: M \rightarrow M_{n \times n} \rightarrow \mathbb{R} \\
& x \mapsto[J f(x)] \mapsto \operatorname{det}([J f(x)])
\end{aligned}
$$

é contínua, existe uma vizinhança $W_{p_{i}}$ de $p_{i}\left(W_{p_{i}} \subset U_{p_{i}}\right)$ tal que $\operatorname{det}(x) \neq 0$ para todo $x \in W_{p_{i}}$, isto é, todos os pontos $x \in W_{p_{i}}$ são pontos regulares de $f, i=1, \ldots, k$.

Tomemos a vizinhança $\tilde{V}=\bigcap_{i=1}^{k} f\left(W_{p_{i}}\right)$ de $p$ em $V$, temos que todo $q \in \tilde{V}$ é um valor regular de $f$. Mas, $\tilde{V}$ é um aberto de $N$, assim $p$ é um ponto interior de $\tilde{V}$ e como $\tilde{V}$ esta 
contido em $R_{1}$ temos que $p$ é um ponto interior de $R_{1}$. Como podemos fazer isto para todo $p \in R_{1}$, segue que $R_{1}$ é aberto.

Consideremos agora o conjunto

$$
R_{2}=\left\{p \in N: f^{-1}(p)=\varnothing\right\}
$$

mostremos que $R_{2}$ é aberto.

Com efeito, suponha que exista algum $p \in R_{2}$ tal que $p$ não é um ponto interior de $R_{2}$, então dada uma vizinhaça aberta $V_{n}$ de $p$ temos $V_{n} \nsubseteq R_{2}$, donde obtemos uma sequência $\left\{y_{n}\right\}$ em $N$, tal que, $y_{n} \rightarrow p$ e $y_{n} \notin R_{2}$, para todo $n \in \mathbb{N}$.

Tomemos então, o compacto

$$
K=\left\{\left\{y_{n}\right\} \cup p\right\}
$$

como $f$ é própria a imagem inversa $f^{-1}(K)=\tilde{K}$ é um compacto de $M$ e como $f$ é contínua, o conjunto $f(\tilde{K})$ é um compacto de $N$, o que é um absurdo, pois $f(\tilde{K})=\left\{y_{n}\right\}$. Logo, todo ponto $p \in R_{2}$ é interior à $R_{2}$, isto é, $R_{2}$ é aberto.

Portanto, o conjunto $R$ é aberto, uma vez que $R=R_{1} \cup R_{2}$.

Até este momento, sabemos que o número $g r_{p}(f)$ depende do valor regular $p$ considerado, porém mediante a hipótese adicional de que a variedade $N$ seja conexa, veremos que $g r_{p}(f)=g r_{q}(f)$, quaisquer que sejam $p$ e $q$ valores regulares de $f$.

Dividiremos a prova deste resultado em três partes, supondo sempre que $p, q \in N$ sejam valores regulares de $f$. Provaremos, primeiramente, que $g r_{p}(f)=g r_{q}(f)$ se $p$ e $q$ são pontos suficientemente "próximos". A seguir, mostraremos que essa igualdade subsiste se $p$ e $q$ pertencem a uma vizinhança coordenada que seja homeomorfa a $\mathbb{R}^{n}$, isto é, se $p, q \in V$ onde $\varphi: V \rightarrow \mathbb{R}^{n}$ é uma carta de $N$ satisfazendo $\varphi(V)=\mathbb{R}^{n}$. Finalmente, demonstraremos que a igualdade continua válida, quaisquer que sejam $p, q \in N$, valores regulares de $f$, desde que $N$ seja uma variedade conexa.

Lema 3.1.6 ([16]). Sejam $M, N$ variedades diferenciáveis $n$-dimensionais, orientadas, com $N$ conexa, e $f: M \rightarrow N$ uma aplicação diferenciável própria. Então, todo valor 
regular $p \in N$ possui uma vizinhança aberta $V$, formada apenas por valores regulares, tais que $g r_{p}(f)=g r_{q}(f)$, para todo $q \in V$.

Demonstração: Faremos a demonstração em dois casos.

Caso 1: Supondo que $f^{-1}(p)=\varnothing$, o resultado segue pelo fato do conjunto

$$
\left\{p \in N: f^{-1}(p)=\varnothing\right\}
$$

ser aberto, como mostramos na Proposição 3.1 .5 .

Caso 2: Consideremos agora o caso em que $p \in N$ é um valor regular de $f$ tal que $f^{-1}(p) \neq \varnothing$, digamos $f^{-1}(p)=\left\{p_{1}, \ldots, p_{r}\right\}$.

Em cada ponto $p_{i} \in f^{-1}(p)$ a aplicação linear

$$
f_{p_{i}}: T_{p_{i}} M \rightarrow T_{p} N
$$

é um isomorfismo. Logo, pelo Teorema da Função Inversa, existem vizinhanças abertas $W_{i}$ de $p_{i}$ em $M$, as quais podemos tomar conexas e duas a duas disjuntas, e $V_{i}$ de $p$ em $N$ tais que

$$
f: W_{i} \rightarrow V_{i}
$$

é um difeomorfismo. Como $W_{i}$ é conexa, todos os pontos de $W_{i}$ tem o mesmo "sinal" relativamente a $f$.

Mostremos agora, que existe uma vizinhança aberta $V$ de $p$ tal que, para todo $q \in V$, o conjunto $f^{-1}(q)$ consta precisamente de $r$ pontos $q_{1}, \ldots, q_{r}$, com $q_{i} \in W_{i}$, isto é, $q_{i}=$ $f^{-1}(q) \cap W_{i}$. Tomando então, uma vizinhança $V$ de $p$ contida em $V_{1} \cap \ldots \cap V_{r}$, veremos que, para todo $q \in V$ e todo $W_{i}$, o conjunto $f^{-1}(q) \cap W_{i}$ é não vazio.

Como $f$ é biunívoca em $W_{i}$, a interseção $f^{-1}(q) \cap W_{i}$ constará de um único ponto $q_{i}$, mas $f^{-1}(q)$ poderá conter outros pontos fora de

$$
W=W_{1} \cup \ldots \cup W_{r}
$$

então devemos mostrar que existe uma vizinhança $V$ de $p$ contida em $V_{1} \cap \ldots \cap V_{r}$, com $f^{-1}(V) \subset W$. 
Suponhamos, por absurdo, que tal $V$ não exista, isto é, suponhamos que para toda vizinhança aberta $V$ de $p$, contida em $V_{1} \cap \ldots \cap V_{r}$ temos $f^{-1}(V) \nsubseteq W$, consideremos $\phi: U \rightarrow \mathbb{R}^{n}$ uma carta de $N$, tal que, $V \subset U$ e $\phi(p)=0$, assim $\phi(V)=A$ é um aberto de $\mathbb{R}^{n}$ contendo a origem.

Para cada $n \in \mathbb{N}$ consideremos a bola aberta $B_{\frac{1}{n}}(0)$, de centro 0 e raio $\frac{1}{n}$, em $\mathbb{R}^{n}$. Logo,

$$
\phi^{-1}\left(B_{\frac{1}{n}}(0) \cap A\right)
$$

é uma vizinhança de $p$ em $N$ e pela nossa hipótese,

$$
f^{-1}\left(\phi^{-1}\left(B_{\frac{1}{n}}(0) \cap A\right)\right) \nsubseteq W \text {. }
$$

Então, existe

$$
x_{n} \in f^{-1}\left(\phi^{-1}\left(B_{\frac{1}{n}}(0) \cap A\right)\right)
$$

tal que $x_{n} \notin W$, e assim obtemos uma sequência $\left\{x_{n}\right\}$, onde $x_{n} \notin W$ para todo $n \in \mathbb{N}$.

Defina agora, a sequência $\left\{y_{n}\right\}$, colocando $y_{n}=f\left(x_{n}\right)$ para todo $n \in \mathbb{N}$, como $\phi\left(f\left(x_{n}\right)\right) \in B_{\frac{1}{n}}(0)$ temos que $y_{n} \rightarrow p$ quando $n \rightarrow \infty$, seja $U_{1}$ uma vizinhança de $p$, cujo fecho $\overline{U_{1}}$ é compacto. Como $y_{n} \rightarrow p$, podemos supor que $y_{n} \in \overline{U_{1}}$ para todo $n \in \mathbb{N}$, assim cada $x_{n} \in f^{-1}\left(\overline{U_{1}}\right)$.

Como a aplicação $f$ é própria, $f^{-1}\left(\overline{U_{1}}\right)$ é um compacto, passando a uma subsequência, se necessário, podemos admitir que $x_{n} \rightarrow x \in M$, assim

$$
f(x)=f\left(\lim _{n \rightarrow \infty} x_{n}\right)=\lim _{n \rightarrow \infty} f\left(x_{n}\right)=p .
$$

Segue então, que $x=p_{i}$ para algum $i=1, \ldots, r$, onde $x \in W$, como $W$ é aberto e $x_{n} \rightarrow x$, segue que $x_{n} \in W$ para todo $n$ suficientemente grande, contradizendo a construção, segundo a qual tomamos $x_{n} \notin W$ para todo $n$.

Portanto, a vizinhança $V \operatorname{com} f^{-1}(V) \subset W$ de fato existe, e o lema esta demonstrado. 
Lema 3.1.7 ([14]). Sejam $M, N$ variedades diferenciáveis $n$-dimensionais, orientadas, com $N$ conexa, e $f: M \rightarrow N$ uma aplicação própria de classe $C^{1}$. Dados $p$ e $q$ valores regulares de $f$ que pertencem a um mesmo aberto $V \subset N$, que é homeomorfo a $\mathbb{R}^{n}$ através de uma carta, então $g r_{p}(f)=g r_{q}(f)$.

Demonstração: Pelo Teorema 1.1.12 existe $\varphi: V \rightarrow \mathbb{R}^{n}$ uma carta de $N$, tal que $p, q \in V$ $\operatorname{com} \varphi(V)=\mathbb{R}^{n}$, podemos supor, sem perda de generalidade, que $\varphi(p)=0$.

Sejam $\varphi(q)=a, v$ o vetor $\overrightarrow{0 a}=a-0$ e $\Delta$ a reta $\{t v: t \in \mathbb{R}\}$, consideremos a aplicação $g=\varphi \circ f: M \rightarrow \mathbb{R}^{n}$ e definamos $\tilde{g}: M \times \mathbb{R} \rightarrow \mathbb{R}^{n}$ colocando $\tilde{g}(p, t)=g(p)+t v$. De acordo com o Teorema 1.1.19, a aplicação $g$ é transversal a reta $\Delta$ se, e somente se, 0 é um valor regular de $\tilde{g}$.

$\operatorname{Em} \varphi(W)$, que é uma vizinhança aberta de $0 \mathrm{em} \mathbb{R}^{n}$, tomemos um ponto $b$ que seja valor regular de $\tilde{g}$, o Teorema de Sard assegura-nos que tal ponto existe. Seja $c=\varphi^{-1}(b) \in W$, nessas condições, $g r_{c}(f)=g r_{p}(f)$, e daqui em diante basta provarmos que $g r_{q}(f)=g r_{c}(f)$.

Podemos supor que 0 é valor regular de $\tilde{g}$, então, de acordo com o Teorema 1.1.19, a aplicação $g$ é transversal a reta $\Delta$, e do Teorema 1.1 .17 segue que $g^{-1}(\Delta)$ é uma subvariedade 1-dimensional de $M$.

Seja $\pi: \mathbb{R}^{n} \rightarrow \mathbb{R}^{n}$ a projeção ortogonal de $\mathbb{R}^{n}$ sobre o hiperplano perpendicular a reta $\Delta$ na origem, se $u=\left(\alpha_{1}, \ldots, \alpha_{n}\right)$ é o vetor unitário de $v=\overrightarrow{0 a}=a-0$, obtemos

$$
\pi\left(x_{1}, \ldots, x_{n}\right)=\left(x_{1}, \ldots, x_{n}\right)-\left(\sum_{i=1}^{n} \alpha_{i} x_{i}\right)\left(\alpha_{1}, \ldots, \alpha_{n}\right)
$$

A imagem $\pi\left(\mathbb{R}^{n}\right)$ é o conjunto

$$
\left\{\left(x_{1}, \ldots, x_{n}\right) \in \mathbb{R}^{n}: \sum_{i=1}^{n} \alpha_{i} x_{i}=0\right\}
$$

que é justamente o hiperplano descrito acima.

Escolhendo uma base nesse hiperplano, podemos identificá-lo com $\mathbb{R}^{n-1}$, definamos a aplicação $\psi=\pi \circ g: M \rightarrow \mathbb{R}^{n-1}$ e observemos que

$$
(\pi \circ g)^{-1}(0)=g^{-1}\left(\pi^{-1}(0)\right)=g^{-1}(\Delta) .
$$


Notemos também que a projeção $\pi$ é uma transformação linear, e pode ser identificada com a sua derivada $d \pi_{x}$ em cada ponto $x \in \mathbb{R}^{n}$, isto é, $d \pi_{x}=\pi$. Como $\pi$ é sobrejetora sobre $\mathbb{R}^{n-1}$, o ponto $0 \in \mathbb{R}^{n-1}$ é um valor regular de $\psi$.

Após essas observações, vamos mostrar que sobre a subvariedade $g^{-1}(\Delta) \subset M$ é possível definir $n-1$ campos diferenciáveis de vetores linearmente independentes, ao mesmo tempo tangentes a variedade $M$ e normais a subvariedade $g^{-1}(\Delta)$.

Seja $p \in g^{-1}(\Delta)$, reconsideremos a aplicação

$$
\psi=\pi \circ g: M \rightarrow \mathbb{R}^{n-1}
$$

que se exprime por meio de $n-1$ funções coordenadas

$$
\psi_{i}\left(x_{1}, \ldots, x_{k}\right)=y_{i}, \quad i=1, \ldots, n-1
$$

onde $k \geq n$ é a dimensão do espaço euclidiano $\mathbb{R}^{k}$ que contém a variedade $M$. Consideremos as aplicações $d \psi_{1_{p}}, \ldots, d \psi_{n-1}$, que são formas lineares sobre o espaço vetorial $T_{p} M$. Por ser euclidiano, o espaço vetorial $T_{p} M$ é isomorfo ao seu dual $T_{p} M^{*}$, esse isomorfismo é

$$
I: T_{p} M \rightarrow T_{p} M^{*}
$$

definido da seguinte forma: para cada $v \in T_{p} M$ colocamos $I(v)=T \in T_{p} M^{*}$ tal que $T(w)=\langle v, w\rangle$, onde $w \in T_{p} M$ e $\langle.,$.$\rangle denota o produto interno usual de \mathbb{R}^{k}$ restrito ao subespaço vetorial $T_{p} M$.

Sejam, $\nabla \psi_{1}, \ldots, \nabla \psi_{n-1}$ os vetores de $T_{p} M$ correspondentes, nesse isomorfismo, às formas $d \psi_{1 p}, \ldots, d \psi_{n-1}$, esses vetores são normais a subvariedade $g^{-1}(\Delta) \subset M$ no ponto $p$, de fato, se $u$ é um vetor tangente a $g^{-1}(\Delta)$ no ponto $p$, resulta

$$
u . \nabla \psi_{i p}=d \psi_{p}(u)=0
$$

pois $\psi_{i}$ é constante sobre $g^{-1}(\Delta)$. 
Como $p \in \psi^{-1}(0)=g^{-1}(\Delta)$ e 0 é valor regular de $\psi$, a matriz jacobiana $[J \psi(p)]_{n-1 \times k}$ tem posto $n-1$, mas $d \psi_{1_{p}}, \ldots, d \psi_{n-1_{p}}$ são exatamente as linhas de $[J \psi(p)]$, logo

$$
d \psi_{1 p}, \ldots, d \psi_{n-1} p
$$

são linearmente independentes, consequentemente os $n-1$ vetores $\nabla \psi_{1}, \ldots, \nabla \psi_{n-1}$ são linearmente independentes.

O conjunto $g^{-1}(\Delta)$ é uma subvariedade 1-dimensional da variedade $M, \operatorname{logo}, g^{-1}(\Delta)$ é uma reunião de curvas abertas e fechadas, que contém os conjuntos $g^{-1}(0)=f^{-1}(p)=$ $\left\{p_{1}, \ldots, p_{r}\right\}$ e $g^{-1}(a)=f^{-1}(q)=\left\{q_{1}, \ldots, q_{s}\right\}$.

Agora, vamos restringir nossas considerações ao segmento $\overline{0 a} \subset \Delta$. Como $f$ é própria e $\varphi$ é um homeomorfismo, a aplicação $g=\varphi \circ f$ também é própria, logo $g^{-1}(\overline{0 a})$ é uma reunião finita de curvas fechadas e curvas abertas com ponto final e inicial bem definidos. Os pontos $p_{1}, \ldots, p_{r}, q_{1}, \ldots, q_{s}$ são necessariamente as extremidades das curvas abertas.

De fato, tomemos uma curva aberta contida em $g^{-1}(\overline{0 a})$ e seja $\gamma:[0,1] \rightarrow M$ uma parametrização desta curva. Suponhamos que $p_{j}$ pertença ao interior de $\gamma([0,1])$, como $p_{j}$ é ponto regular de $g$ a aplicação linear $d g_{p_{j}}: T_{p_{j}} M \rightarrow \mathbb{R}^{n}$ é um isomorfismo, então pelo Teorema da Aplicação Inversa, existem vizinhanças abertas $U_{p_{j}}$ de $p_{j}$ em $M$ e $V_{0}$ de 0 em $\mathbb{R}^{n}$ tais que a aplicação $g: U_{p_{j}} \rightarrow V_{0}$ é um difeomorfismo local. Denotemos por $W$ o conjunto

$$
\left(\left(U_{p_{j}} \cap \gamma([0,1])\right) \backslash\left\{p_{j}\right\}\right)
$$

Então, $\left.g\right|_{W}: W \rightarrow g(W)$ também é um difeomorfismo, o que é um absurdo pois $W$ é desconexo e $g(W)$ é conexo.

Analogamente, mostramos que $p_{j}$ não pode pertencer a nenhuma curva fechada. Portanto, para efeito de avaliação de $g r_{p}(f)$ e de $g r_{q}(f)$, as curvas fechadas eventualmente contidas em $g^{-1}(\overline{0 a})$ podem ser desconsideradas, já que não contém pontos de $f^{-1}(p)$, nem de $f^{-1}(q)$.

Resta fazermos um estudo cuidadoso relativo as curvas abertas.

Tomemos uma curva aberta qualquer, que denotaremos por $S$, cujas extremidades representaremos por $A, B$, e seja $c:[0,1] \rightarrow M$ uma parametrização diferenciável desta 
curva, tal que $c(0)=A, c(1)=B$ e $c(t)=p$. Chamemos $e_{1}, \ldots, e_{n-1}$ aos $n-1$ campos de vetores contínuos cuja existência há pouco demonstramos. No ponto $p=c(t)$, os vetores $e_{1}(t), \ldots, e_{n-1}(t)$ são, como vimos, tangentes a variedade $M$ e normais a $S=c([0,1])$.

Seja $e_{n}(t)$ o vetor $c^{\prime}(t)$, tangente a $S$ no ponto $p$. Então, o conjunto

$$
\left\{e_{1}(t), \ldots, e_{n-1}(t), e_{n}(t)\right\}
$$

constitui uma base do espaço vetorial tangente $T_{p} M$.

Podemos admitir que essa base seja compatível com a orientação existente em $T_{p} M$ como espaço tangente a variedade orientada $M$, pois se não a for basta substituírmos um dos $n-1$ primeiro vetores pelo seu simétrico e obteremos uma base na dita condição.

Consideremos o determinante

$$
D(t)=\operatorname{det}\left(d g_{p}\left(e_{1}(t)\right), \ldots, d g_{p}\left(e_{n-1}(t)\right), d g_{p}\left(e_{n}(t)\right)\right)
$$

onde $d g_{p}\left(e_{i}(t)\right)$ indica a coluna formada pelas $n$ componentes do vetor $d g_{p}\left(e_{i}(t)\right) \in \mathbb{R}^{n}$. Se $u$ é o vetor unitário de $\vec{v}=a-0$, podemos escrever

$$
d g_{p}\left(e_{n}(t)\right)=d g_{p}\left(c^{\prime}(t)\right)=\lambda(t) u
$$

onde $\lambda(t)$ é uma função real contínua.

Consideremos também o determinante

$$
A(t)=\operatorname{det}\left(d g_{p}\left(e_{1}(t)\right), \ldots, d g_{p}\left(e_{n-1}(t)\right), u\right) .
$$

Em virtude da transversalidade de $g$ sobre a reta $\Delta$, segue que $A(t) \neq 0$, para todo $t \in[0,1]$. Como $A(t)$ depende continuamente de $t$, podemos afirmar que $A(t)$ não muda de sinal quando $t$ percorre o intervalo $[0,1]$. Observemos que entre $D(t)$ e $A(t)$ existe a seguinte relção

$$
D(t)=\lambda(t) A(t) .
$$


Como os pontos $A$ e $B$ são elementos do conjunto

$$
f^{-1}(p) \cup f^{-1}(q)=\left\{p_{1}, \ldots, p_{s}\right\} \cup\left\{q_{1}, \ldots, q_{r}\right\}
$$

pode acontecer que $A$ pertença a um dos conjuntos $f^{-1}(p), f^{-1}(q)$ e $B$ pertença ao outro, mas pode também suceder que $A$ e $B$ estejam ambos num só desses conjuntos. Estudemos as duas possibilidades.

Suponhamos, primeiramente, que $A \in f^{-1}(p)$ e $B \in f^{-1}(q)$, então, $A=p_{i}$ e $B=q_{j}$ e temos $g(A)=0, g(B)=a$, como os pontos $p_{i}$ e $q_{j}$ são pontos regulares de $g$ existem vizinhanças de $p_{i}$ e $q_{j}$ em $M$, que chamaremos de $V_{p_{i}}$ e $V_{q_{j}}$, respectivamente.

As vizinhanças $V_{p_{i}}$ e $V_{q_{j}}$ se aplicam difeomorficamente sobre vizinhanças de 0 e de $a$ em $\mathbb{R}^{n}$, dessa forma, podemos afirmar que para valores não nulos de $t$ suficientemente próximos de 0 , os correspondentes pontos $g(p)=g(c(t))$ são vizinhos de 0 , mas não nulos.

De modo análogo se comportam os pontos $g(p)=g(c(t))$ que correspondem a valores de $t$ bastante próximos de 1 , e diferentes de 1 .

Considerando $\overline{0 a}$ como o segmento orientado de origem 0, seja a função

$$
h(t)=(g(c(t))-0) \cdot u
$$

onde $\vec{u}$ é o vetor unitário de $\vec{v}=a-0$ e o ponto indica o produto escalar. Derivando a função $h$, obtemos

$$
h^{\prime}(t)=\left((g \circ c)^{\prime}(t)\right) \cdot \vec{u}=\left(d g_{p}\left(c^{\prime}(t)\right)\right) \cdot \vec{u}=\lambda(t) \vec{u} \cdot \vec{u}=\lambda(t)
$$

Agora, tomemos as vizinhanças $V_{p_{i}, S}=V_{p_{i}} \cap S$ e $V_{q_{j}, S}=V_{q_{j}} \cap S$ dos pontos $p_{i}$ e $q_{j}$, respectivamente, em $S$. Consideremos os pontos da forma $c(t)=p$ pertencentes a $V_{p_{i}, S} \mathrm{e}$ a $V_{q_{j}, S}$, como $c^{\prime}(t)=e_{n}(t)$ é uma base para o espaço vetorial tangente a subvariedade $S$, e como $\left.d g_{p}\right|_{T_{p} S}$ é um isomorfismo, temos que $\left.d g_{p}\right|_{T_{p} S}\left(e_{n}(t)\right)=d g_{p}\left(e_{n}(t)\right)=\lambda(t) u$ é uma base para o espaço vetorial tangente ao segmento $\overline{0 a}$ em $g(c(t))$.

Os pontos da forma $c(t)=p$, pertencentes a $V_{p_{i}, S}$ e a $V_{q_{j}, S}$, são sempre positivos ou sempre negativos em relação ao isomorfismo $\left.d g_{p}\right|_{T_{p} S}$, pois as vizinhanças $V_{p_{i}, S}$ e $V_{q_{j}, S}$ são 
conexas. Suponhamos que estes pontos sejam negativos, então $\lambda(t)<0$ para todo $t$ tal que $c(t)$ pertence a $V_{p_{i}, S}$ e a $V_{q_{j}, S}$, o que significa que $h$ é decrescente a direita de 0 e também a esquerda de 1, mas isso é um absurdo, pelas considerações que fizemos acima.

Concluímos assim, que $\lambda(0)>0$ e $\lambda(1)>0$, segue disto e da Equação 3.1 .1 que $D(0)$ tem o mesmo sinal que $A(0)$ e que $D(1)$ tem o mesmo sinal que $A(1)$. Mas, já vimos que $A(0)$ e $A(1)$ tem o mesmo sinal, portanto o mesmo ocorre com $D(0)$ e $D(1)$, e isso significa que os pontos $p_{i}$ e $q_{j}$ são ambos positivos ou ambos negativos.

Admitamos agora, que $A, B \in f^{-1}(p)$, por exemplo, então $A=p_{i}, B=p_{j}$, onde $i \neq j$. Neste caso, $g(A)=g(B)=0$ e empregando a mesma função real $h$ definida anteriormente e utilizando o mesmo argumento que antes, verificamos que $\lambda(0)>0$ e $\lambda(1)<0$. Assim, da Equação 3.1.1 temos que $D(0)$ e $A(0)$ tem o mesmo sinal, ao passo que $D(1)$ e $A(1)$ tem sinais contrários, como $A(0)$ e $A(1)$ tem o mesmo sinal, segue que $D(0)$ e $D(1)$ tem sinais contrários. Logo se $p_{i}$ é positivo, $p_{j}$ é negativo, e vice-versa.

Assim, se a curva aberta $S$ esta no segundo caso, ela não pesa no cálculo de $g r_{p}(f)$, ou de $g r_{q}(f)$, pois suas extremidades são pontos de $f^{-1}(p)$, ou $f^{-1}(q)$, de sinais contrários. As únicas curvas que influenciam no cálculo dos graus são as que estão no primeiro caso. Para estas, as extremidades são ambas positivas ou ambas negativas, e uma se aplica pela $f$ em $p$ e a outra em $q$. Portanto, $g r_{p}(f)=g r_{q}(f)$, como queríamos demonstrar.

Teorema 3.1.8 ([16]). Sejam $M, N$ variedades diferenciáveis $n$-dimensionais, orientadas, com $N$ conexa, e $f: M \rightarrow N$ uma aplicação própria de classe $C^{1}$. Então, o grau $g r_{p}(f)$ independe do valor regular $p \in N$.

Demonstração: Dada $f: M \rightarrow N$ uma aplicação própria de classe $C^{1}$, consideremos $p, q \in N$ valores regulares de $f$. Sendo $N$ conexa e como toda variedade diferenciável é localmente conexa, segue que $N$ é conexa por caminhos. Logo, existe um caminho $\alpha:[0,1] \rightarrow N$ ligando os pontos $p$ e $q$.

Agora, como $\alpha([0,1])$ é compacto, dada uma cobertura de $\alpha([0,1])$ por vizinhanças coordenadas (tal cobertura existe, pois $N$ é variedade diferenciável) conseguimos uma subcobertura $\left\{V_{1}, \ldots, V_{s}\right\}$ finita de $\alpha([0,1])$ por vizinhanças coordenadas. 
Considerando essa subcobertura, $\left\{V_{1}, \ldots, V_{s}\right\}$ e chamando $p=a_{0}$ e $q=a_{s}$ podemos tomar pontos $a_{1}, \ldots, a_{s-1}$, tais que $a_{i-1}$ e $a_{i}$ estejam na mesma vizinhança coordenada $V_{i}$, para $i=1, \ldots, s$.

Como $N$ é conexa podemos considerar que cada vizinhança $V_{i}$ é difeomorfa a $\mathbb{R}^{n}$, além disso, deslocando ligeiramente $a_{i-1}$ e $a_{i}$, se necessário for, sem sair de $V_{i}$, podemos supor que os $a_{i}$ 's são valores regulares de $f$, em virtude do Teorema de Sard.

Considerando $\varphi: V_{i} \rightarrow \mathbb{R}^{n}$ o difeomorfismo entre $V_{i}$ e o espaço euclidiano $\mathbb{R}^{n}$, e chamando $b_{i-1}=\varphi\left(a_{i-1}\right)$ e $b_{i}=\varphi\left(a_{i}\right)$, pelo Lema 3.1 .7 temos

$$
g r_{b_{i-1}}(\varphi \circ f)=g r_{b_{i}}(\varphi \circ f)
$$

Mas, $V_{i}$ é conexa e $\varphi$ é um difeomorfismo, então todo ponto $v \in V_{i}$ tem o mesmo sinal relativamente a $\varphi$. Logo,

$$
g r_{a_{i}}(f)=g r_{a_{i-1}}(f), \quad i=1, \ldots, s
$$

Portanto, $g r_{p}(f)=g r_{q}(f)$.

A conexidade da variedade $N$ é uma hipótese essencial para o Teorema 3.1.8, vejamos, através do exemplo abaixo, que quando a variedade do contra domínio não é conexa o grau de $f$ relativamente a um valor regular pode assumir valores diferentes.

Exemplo 3.1.9. Consideremos a função $f:(0,1) \rightarrow(-1,0) \cup(0,1)$ dada por $f(x)=x^{2}$. Assim, temos dois casos a considerar:

(i) Se $h<0$, então $f^{-1}(h)=\varnothing$ e consequentemente $g r_{h}(f)=0$;

(ii) Se $h>0$, então $f^{-1}(h)=\{\sqrt{h}\}$ e como vimos no Exemplo $3.1 .2 h$ é positivo. Logo $g r_{h}(f)=1$.

Tão importante quanto a conexidade da variedade $N$ no Teorema 3.1 .8 são as hipóteses de que a aplicação $f: M \rightarrow N$ é própria e que as variedades $M$ e $N$ consideradas não possuem bordo. Vejamos os seguintes exemplos. 
Exemplo 3.1.10. Consideremos a função $f:(0,1) \rightarrow \mathbb{R}$ dada por $f(x)=x^{2}$. E observemos que tal função não é própria, pois $f^{-1}([0,1])=(0,1)$, que não é compacto.

Observemos também que se $h<0$ ou $h \geq 1$, temos que $f^{-1}(h)=\varnothing$ e consequentemente $g r_{h}(f)=0$, por outro lado, se $h \in(0,1)$, então $f^{-1}(h)=\{\sqrt{h}\}$ e como vimos no Exemplo $3.1 .2 h$ é positivo, $\operatorname{logo} g r_{h}(f)=1$.

Exemplo 3.1.11. Consideremos a função $f:[0,1) \rightarrow(-1,1)$ dada por $f(x)=x^{2}$. E observemos que a variedade $[0,1)$ possue bordo.

Observemos também que se $h<0$, temos que $f^{-1}(h)=\varnothing$ e consequentemente $g r_{h}(f)=0$, por outro lado, se $h \in(0,1)$, então $f^{-1}(h)=\{\sqrt{h}\}$ e como vimos no Exemplo 3.1.2 $h$ é positivo, $\operatorname{logo} g r_{h}(f)=1$.

Em virtude do Teorema 3.1.8, podemos agora definir o grau de uma aplicação $f: M \rightarrow$ $N$ própria de classe $C^{1}$.

Definição 3.1.12. Dadas $M$ e $N$ duas variedades diferenciáveis $n$-dimensionais, orientadas, com $N$ conexa, e $f: M \rightarrow N$ uma aplicação própria de classe $C^{1}$, chamamos de grau de $f$ o número,

$$
g r(f)=g r_{p}(f)
$$

onde $p \in N$ é um valor regular qualquer da aplicação $f$.

Como consequência do Teorema 3.1 .8 temos o seguinte resultado.

Proposição 3.1.13. Sejam $M, N$ variedades diferenciáveis $n$-dimensionais, orientadas, com $N$ conexa. Dada $f: M \rightarrow N$ uma aplicação própria de classe $C^{1}$, se $g r(f) \neq 0$, então $f$ é sobrejetora.

Demonstração: Com efeito, suponha que exista $y \in f(M)^{c}$, por definição, $y$ será valor regular de $f$. Como $f^{-1}(y)=\varnothing$, temos $g r(f)=0$, o que é um absurdo.

Da Proposição 3.1.13, segue que dadas $M, N$ variedades diferenciáveis $n$-dimensionais, orientadas, com $N$ conexa, e $f: M \rightarrow N$ uma aplicação diferenciável própria que não seja sobrejetora, então $\operatorname{gr}(f)=0$. 
Exemplo 3.1.14. Sejam $M$ uma variedade diferenciável $n$-dimensional, compacta e orientada, e $f: M \rightarrow \mathbb{R}^{n}$ uma aplicação própria de classe $C^{1}$.

Como $M$ é compacta e $f$ é uma aplicação contínua, temos que $f(M)$ é um subconjunto compacto de $\mathbb{R}^{n}$, logo, $f(M)$ é um subconjunto fechado e limitado de $\mathbb{R}^{n}$. Portanto, a aplicação $f$ não é sobrejetora, consequentemente $g r(f)=0$.

Porém a recíproca deste fato não é verdadeira, existem aplicações diferenciáveis próprias, definidas entre variedades orientadas de mesma dimensão, que são sobrejetoras cujo grau é igual a 0 .

Exemplo 3.1.15. Seja $f:(-1,0) \cup(0,1) \rightarrow(0,1)$ a aplicação dada por $f(x)=x^{2}$. Temos que $f$ é sobrejetora, além disso, utilizando o Exemplo 3.1 .2 podemos concluir que $g r(f)=0$.

Sejam $M, N$ variedades diferenciáveis $n$-dimensionais, orientadas, com $N$ conexa. Dada $f: M \rightarrow N$ uma aplicação diferenciável própria, dependo da natureza das variedades $M$ e $N$, o cálculo do grau de $f$ pode se tornar muito difícil. Em alguns casos, podemos contornar esta situação com as seguintes propriedades.

Teorema 3.1.16 ([16]). Sejam $M, N$ e $P$ variedades diferenciáveis $n$-dimensionais, orientadas, com $N$ e $P$ conexas. Consideremos, $f: M \rightarrow N$ e $g: N \rightarrow P$ aplicações próprias de classe $C^{1}$, então $g r(g \circ f)=g r(g) g r(f)$.

Demonstração: Sejam $A \subset P$ e $B \subset P$ os conjuntos dos valores regulares de $g$ e $g \circ f$ respectivamente. Como vimos na Proposição 3.1.5, os conjuntos $A$ e $B$ são abertos, segue então do Teorema de Sard, que existe um ponto $p \in P$ que é valor regular de $g$ e $g \circ f$ simultaneamente.

Então, temos dois casos para analisar, se $(g \circ f)^{-1}(p)=\varnothing$, então $g^{-1}(p)=\varnothing$ ou $f^{-1}\left(g^{-1}(p)\right)=\varnothing$, e o resultado segue direto.

No caso em que $(g \circ f)^{-1}(p) \neq \varnothing$, e consideremos $g^{-1}(p)=\left\{p_{1}, \ldots, p_{r}\right\}$, então todos os pontos $p_{i}$ são valores regulares de $f$, pois como $N$ e $P$ tem a mesma dimensão

$$
d g_{p_{i}}: T_{p_{i}} N \rightarrow T_{p} P
$$


é um isomorfismo.

Para cada $p_{i} \in g^{-1}(p)$, coloquemos $f^{-1}\left(p_{i}\right)=\left\{p_{i 1}, \ldots, p_{i s_{i}}\right\}$, então, $(g \circ f)^{-1}(p)=\left\{p_{i j}: i=1, \ldots, r, \quad j=1, \ldots, s_{i}\right\}$, o sinal de $p_{i j}$ relativamente a $g \circ f$ é o produto do sinal de $p_{i j}$ relativamente a $f$ pelo sinal de $p_{i}=f\left(p_{i j}\right)$ relativamente a g. Seja $\varepsilon_{i}$ o sinal de $p_{i}$, isto é, $\varepsilon_{i}=+1$ se $p_{i}$ é positivo e $\varepsilon_{i}=-1$ se $p_{i}$ é negativo. Analogamente, sejam $\eta_{i j}$ o sinal de $p_{i j}$ relativamente a $f$ e $\rho_{i j}$ o sinal de $p_{i j}$ relativamente a $g \circ f$. Temos

$$
g r(g)=\sum_{i=1}^{r} \varepsilon_{i} \text { e } \operatorname{gr}(f)=\sum_{j=1}^{s_{i}} \eta_{i j}
$$

independentemente de $i$, pelo Teorema 3.1.8. Além disso, $\rho_{i j}=\eta_{i j} \cdot \varepsilon_{i} \mathrm{e}$

$$
\begin{aligned}
g r(g \circ f)=\sum_{i, j=1}^{r, s_{i}} \rho_{i j} & =\sum_{i=1}^{r}\left(\sum_{j=1}^{s_{i}} \eta_{i j}\right) \varepsilon_{i} \\
& =\sum_{i=1}^{r} g r(f) \varepsilon_{i} \\
& =\operatorname{gr}(f) \sum_{i=1}^{r} \varepsilon_{i} \\
& =\operatorname{gr}(f) \operatorname{gr}(g) .
\end{aligned}
$$

Do Teorema anterior decorre o seguinte corolário.

Corolário 3.1.17. Sejam $M, N$ variedades diferenciáveis $n$-dimensionais, $P, Q$ variedades diferenciáveis $r$-dimensionais, com $M, N, P, Q$ orientadas e $N, Q$ conexas. Dadas $f: M \rightarrow N$ e $g: P \rightarrow Q$ aplicações próprias de classe $C^{1}$, seja a aplicação

$$
\begin{aligned}
f \times g: M \times P & \rightarrow \quad N \times Q \\
(x, y) & \mapsto \quad(f(x), g(y)) .
\end{aligned}
$$

Então,

$$
g r(f \times g)=g r(f) g r(g) .
$$

Demonstração: Podemos escrever $f \times g=(f \times j) \circ(i \times g)$ onde $i: M \rightarrow M$ e $j: Q \rightarrow Q$ indicam as aplicações identidade. 
Considerando as variedades $M \times P$ e $N \times Q$ munida da orientação produto (ver [16], página 9), é fácil ver que $g r(f \times j)=g r(f)$ e $g r(i \times g)=g r(g)$. O corolário segue então do Teorema 3.1.16.

Exemplo 3.1.18. A aplicação antípoda $\alpha: \mathbb{S}^{n} \rightarrow \mathbb{S}^{n}$, definida por

$$
\alpha\left(x_{1}, \ldots, x_{n+1}\right)=\left(-x_{1}, \ldots,-x_{n+1}\right)
$$

tem grau $(-1)^{n+1}$.

Com efeito, temos $\alpha=f_{1} \circ f_{2} \circ \ldots \circ f_{n+1}$, onde cada

$$
f_{i}: \mathbb{S}^{n} \rightarrow \mathbb{S}^{n}
$$

é dada por $f_{i}\left(x_{1}, \ldots, x_{n+1}\right)=\left(x_{1}, \ldots,-x_{i}, \ldots, x_{n+1}\right)$ e tem grau -1 , em virtudade do Exemplo 3.1.4 então pelo Teorema 3.1.16, concluímos que $\operatorname{gr}(\alpha)=(-1)^{n+1}$.

O Teorema a seguir relaciona os conceitos de homotopia e grau de uma aplicação.

Teorema 3.1.19. Sejam $M, N$ variedades diferenciáveis $n$-dimensionais, orientadas, com $M$ compacta, e $f, g: M \rightarrow N$, aplicações próprias de classe $C^{1}$ que admitem o mesmo ponto $p \in N$ como valor regular. Se $f \simeq g$, então $g r_{p}(f)=g r_{p}(g)$.

Para a demonstração deste resultado veja [16] pg 119.

Vimos que a noção de grau de uma aplicação envolve o conceito de valor regular, é natural, então, pensarmos que este conceito pode ser definido apenas para aplicações diferenciáveis.

Entretanto, pelo Teorema 1.2.6, dada uma aplicação contínua $f: M \rightarrow N$ existe uma aplicação $g: M \rightarrow N$ de classe $C^{1}$ que é homotópica a $f$. Assim, utilizando o Teorema 3.1.19, podemos agora definir o conceito de grau de uma aplicação contínua.

Definição 3.1.20. Sejam $M$ e $N$ variedades diferenciáveis $n$-dimensionais, compactas e orientadas, $f: M \rightarrow N$ uma aplicação contínua, e $g: M \rightarrow N$ uma aplicação de classe $C^{1}$ homotópica a $f$. Definamos o grau de $f$, que denotaremos por $g r(f)$, como sendo o grau da aplicação $g$. 
A seguir apresentaremos dois exemplos, que decorrem do Teorema 3.1.19.

Exemplo 3.1.21. (O Teorema Fundamental da Álgebra) Seja $p: \mathbb{C} \rightarrow \mathbb{C}$ um polinômio de grau $n \geq 1$, então, $p$ é sobrejetor.

De fato, identificando o conjunto dos números complexos $\mathbb{C}$ com $\mathbb{R}^{2}$, a derivada do polinômio $p$ em um ponto $z \in \mathbb{C}$

$$
d p_{z}: \mathbb{R}^{2} \rightarrow \mathbb{R}^{2}
$$

é uma transformação linear que consiste na multiplicação por um número complexo. Logo, $d p_{z} \equiv 0$ ou o determinante jacobiano $\operatorname{det}[J p(z)]>0$. Assim, se o ponto $w \in \mathbb{C}$ é um valor regular de $p$, então $g r_{w}(p)$ é o número de elementos em $p^{-1}(w)$.

Sem perda de generalidade, podemos admitir que $p(z)=z^{n}+q(z)$, onde $q$ é um polinômio de grau $\leq n-1$. A aplicação $H: \mathbb{C} \times[0,1] \rightarrow \mathbb{C}$, definida por

$$
H(z, t)=z^{n}+(1-t) q(z)
$$

é uma homotopia entre $p$ e o polinômio $\varphi: \mathbb{C} \rightarrow \mathbb{C}$, dado por $\varphi(z)=z^{n}$.

Para todo $a \neq 0$ em $\mathbb{C}$, a equação $z^{n}=a$ tem exatamente $n$ raízes, assim $\operatorname{gr}(\varphi)=n$, logo, pelo Teorema $3.1 .19 \operatorname{gr}(p)=n$. Portanto, da Proposição 3.1.13. temos que $p$ é sobrejetor.

Exemplo 3.1.22. Seja $\overline{\mathbb{B}}_{1}(0) \subset \mathbb{R}^{n+1}$ a bola fechada centrada em $0 \in \mathbb{R}^{n}$ e de raio 1. Como vimos na Proposição 1.2.5, uma aplicação contínua $f: \mathbb{S}^{n} \rightarrow \mathbb{S}^{n}$ estende-se continuamente a uma aplicação $\tilde{f}: \overline{\mathbb{B}}_{1}(0) \rightarrow \mathbb{S}^{n}$ se, e somente se, $f$ é homotópica a uma constante.

Portanto, do Teorema 3.1.19, uma aplicação contínua $f: \mathbb{S}^{n} \rightarrow \mathbb{S}^{n}$ estende-se continuamente a uma aplicação $\tilde{f}: \overline{\mathbb{B}}_{1}(0) \rightarrow \mathbb{S}^{n}$ se, e somente se, $g r(f)=0$.

Teorema 3.1.23. Seja $M$ uma variedade diferenciável $n$-dimensional, compacta, conexa e orientada, e seja $\mathbb{S}^{n}$ uma esfera de mesma dimensão que $M$. Se duas aplicações $f, g: M \rightarrow \mathbb{S}^{n}$ tem o mesmo grau, então $f$ e $g$ são homotópicas.

Para a demonstração deste resultado ver [16] pg 68 ou [13] pg 129. 
Sejam $M$ e $N$ variedades diferenciáveis $n$-dimensionais, orientadas, compactas e conexas, como vimos na Seção 1.3, uma aplicação própria $f: M \rightarrow N$ induz um homomorfismo $f_{*}: H_{n}(M) \rightarrow H_{n}(N)$, do grupo de homologia de $M$ no grupo de homologia de $N$. Além disso, podemos mostrar que os grupos de homologia de $M$ e $N$ na dimensão $n, H_{n}(M)$ e $H_{n}(N)$, são isomorfos ao grupo dos inteiros $\mathbb{Z}$.

Como ocorre com todo homomorfismo de $\mathbb{Z}$ em $\mathbb{Z}, f_{*}$ é a multiplicação por um número real, que denotaremos por $r$.

Teorema 3.1.24. Sejam, $M$ e $N$ variedades diferenciáveis $n$-dimensionais, orientadas e conexas, e $f: M \rightarrow N$ uma aplicação própria de classe $C^{1}$, então, $g r(f)=r$.

Para a demonstração do teorema acima, veja [15] (Teorema 21, pg 510).

Utilizando o conceito de grau, na próxima seção, apresentaremos a noção de grau local de uma aplicação contínua, a partir de informações desta aplicação em uma vizinhaça de um dado ponto.

\subsection{Grau local de uma aplicação}

Começaremos esta seção relembrando a definição de tipo de homotopia entre espaços topológicos e apresentando um exemplo deste conceito, o qual utilizaremos no decorrer de toda a seção.

Definição 3.2.1. Sejam $X$ e $Y$ espaços topológicos, dizemos que uma aplicação contínua $f: X \rightarrow Y$ é uma equivalência homotópica quando existe uma aplicação contínua $g$ : $Y \rightarrow X$ tal que $g \circ f \simeq i d: X \rightarrow X$ e $f \circ g \simeq i d: Y \rightarrow Y$. Neste caso, $g$ chama-se uma equivalência inversa de $f$ e os espaços $X$ e $Y$ dizem-se ter o mesmo tipo de homotopia.

Exemplo 3.2.2. Dado um ponto $a \in \mathbb{R}^{n}$, seja $\overline{\mathbb{B}}_{\varepsilon}(a)$ a bola fechada de centro $a$ e raio $\varepsilon>0$ em $\mathbb{R}^{n}$. Então, $\overline{\mathbb{B}}_{\varepsilon}(a) \backslash\{a\}$ e a esfera unitária $\mathbb{S}^{n-1}$ têm o mesmo tipo de homotopia.

De fato, sejam

$$
\rho=\rho_{a, \varepsilon}: \overline{\mathbb{B}}_{\varepsilon}(a) \backslash\{a\} \rightarrow \mathbb{S}^{n-1} \quad \text { e } \quad j=j_{a, \varepsilon}: \mathbb{S}^{n-1} \rightarrow \overline{\mathbb{B}}_{\varepsilon}(a) \backslash\{a\}
$$


as aplicações definidas por

$$
\rho(x)=\frac{x-a}{\|x-a\|} \text { e } j(y)=a+\varepsilon y
$$

assim, $\rho \circ j=i d: \mathbb{S}^{n-1} \rightarrow \mathbb{S}^{n-1}$. Além disso, considerando a aplicação contínua $F: \overline{\mathbb{B}}_{\varepsilon}(a) \backslash\{a\} \times I \rightarrow \overline{\mathbb{B}}_{\varepsilon}(a) \backslash\{a\}$ dada por

$$
F(x, t)=(1-t)\left(a+\varepsilon \frac{x-a}{\|x-a\|}\right)+t x .
$$

temos $j \circ \rho \simeq i d: \overline{\mathbb{B}}_{\varepsilon}(a) \backslash\{a\} \rightarrow \overline{\mathbb{B}}_{\varepsilon}(a) \backslash\{a\}$.

Fazendo $\varepsilon=\infty$ no Exemplo 3.2.2, obtemos $\mathbb{B}=\mathbb{R}^{n}$. Continuamos tendo $\mathbb{R}^{n} \backslash\{a\}$ e $\mathbb{S}^{n-1}$ do mesmo tipo de homotopia, a equivalência homotópica

$$
\rho=\rho_{a, \infty}: \mathbb{R}^{n} \backslash\{a\} \rightarrow \mathbb{S}^{n-1}
$$

define-se do mesmo modo, mas sua inversa $j=j_{a, \infty}: \mathbb{S}^{n-1} \rightarrow \mathbb{R}^{n} \backslash\{a\}$ é definida por $j(y)=a+y$.

Definição 3.2.3. Sejam $U \subset \mathbb{R}^{n}$ um aberto, $a \in U, b \in \mathbb{R}^{n}$, e $f: U \backslash\{a\} \rightarrow \mathbb{R}^{n} \backslash\{b\}$ uma aplicação contínua. Consideremos $\overline{\mathbb{B}}_{\varepsilon}(a) \subset U$ uma bola fechada de centro $a$ e raio $\varepsilon$ e as aplicações $j=j_{a, \varepsilon}: \mathbb{S}^{n-1} \rightarrow \overline{\mathbb{B}}_{\varepsilon}(a) \backslash\{a\}, \rho=\rho_{b, \infty}: \mathbb{R}^{n} \backslash\{b\} \rightarrow \mathbb{S}^{n-1}$, introduzidas acima.

Consideremos também a composição $\rho \circ f \circ j: \mathbb{S}^{n-1} \rightarrow \mathbb{S}^{n-1}$, definimos o grau local da aplicação $f$ em $a$, que denotaremos por $\gamma_{a}(f)$, como sendo o grau da aplicação $\rho \circ f \circ j$ : $\mathbb{S}^{n-1} \rightarrow \mathbb{S}^{n-1}$, isto é, $\gamma_{a}(f)=\operatorname{gr}(\rho \circ f \circ j)$.

Observemos que $\gamma_{a}(f)$ não depende da escolha da bola fechada $\overline{\mathbb{B}}_{\varepsilon}(a) \subset U$. De fato, seja $\overline{\mathbb{B}}_{\varepsilon_{1}}(a) \subset U$ a bola fechada de centro em $a$ e raio $\varepsilon_{1}>0$, suponhamos que $\varepsilon_{1} \leq \varepsilon$, então $\overline{\mathbb{B}}_{\varepsilon_{1}}(a) \subset \overline{\mathbb{B}}_{\varepsilon}(a)$, seja

$$
k: \overline{\mathbb{B}}_{\varepsilon_{1}}(a) \backslash\{a\} \rightarrow \overline{\mathbb{B}}_{\varepsilon}(a) \backslash\{a\}
$$


a inclusão. Tomando a aplicação $J: \mathbb{S}^{n-1} \times I \rightarrow \overline{\mathbb{B}}_{\varepsilon}(a) \backslash\{a\}$ dada por

$$
J(y, t)=(1-t)\left(a+\varepsilon_{1} y\right)+t(a+\varepsilon y)
$$

vemos que $k \circ j^{1} \simeq j: \mathbb{S}^{n-1} \rightarrow \overline{\mathbb{B}}_{\varepsilon}(a) \backslash\{a\}$. Como

$$
f \circ k: \overline{\mathbb{B}}_{\varepsilon_{1}}(a) \backslash\{a\} \rightarrow \mathbb{R}^{n} \backslash\{b\}
$$

é a restrição de $f$ a $\overline{\mathbb{B}}_{\varepsilon_{1}}(a) \backslash\{a\}$, o grau local da aplicação $f$, definido por meio da bola $\overline{\mathbb{B}}_{\varepsilon_{1}}(a)$ em vez de $\overline{\mathbb{B}}_{\varepsilon}(a)$, é o grau da aplicação

$$
\rho \circ f \circ k \circ j^{1}: \mathbb{S}^{n-1} \rightarrow \mathbb{S}^{n-1}
$$

Mas $\rho \circ f \circ k \circ j^{1} \simeq \rho \circ f \circ j$. Portanto, pelo Teorema 3.1.19, $g r\left(\rho \circ f \circ k \circ j^{1}\right)=g r(\rho \circ f \circ j)$.

Observação 3.2.4. 1) O grau local $\gamma_{a}(f)$ depende somente do comportamento de $f$ numa vizinhança arbitrariamente pequena do ponto a. Mais precisamente, se $U, V \subset$ $\mathbb{R}^{n}$ são abertos contendo o ponto a, e

$$
f: U \backslash\{a\} \rightarrow \mathbb{R}^{n} \backslash\{b\}, \quad g: V \backslash\{a\} \rightarrow \mathbb{R}^{n} \backslash\{b\}
$$

são aplicações contínuas tais que $\left.f\right|_{W}=\left.g\right|_{W}$, onde $W$ é um aberto de $\mathbb{R}^{n}$ satisfazendo $W \subset U \cap V$, então $\gamma_{a}(f)=\gamma_{a}(g)$. Basta, na definição de grau local, tomar a bola $\overline{\mathbb{B}}$ tão pequena que $\overline{\mathbb{B}} \subset W$ e usá-la para calcular $\gamma_{a}(f)$ e $\gamma_{a}(g)$.

2) Se $f \simeq g: U \backslash\{a\} \rightarrow \mathbb{R}^{n} \backslash\{b\}$, então $\gamma_{a}(f)=\gamma_{a}(g)$. Com efeito, temos $f \simeq g$ : $\overline{\mathbb{B}} \backslash\{a\} \rightarrow \mathbb{R}^{n} \backslash\{b\}$, donde $\rho \circ f \circ j \simeq \rho \circ g \circ j$ e dai

$$
\gamma_{a}(f)=\operatorname{gr}(\rho \circ f \circ j)=\operatorname{gr}(\rho \circ g \circ j)=\gamma_{a}(g)
$$


Exemplo 3.2.5. Consideremos cada ponto $z=(x, y) \in \mathbb{R}^{2}$ como um número complexo $z=x+i y$, e a aplicação contínua

$$
\begin{aligned}
f: \mathbb{R}^{2} \backslash\{0\} & \rightarrow \mathbb{R}^{2} \backslash\{0\} \\
z & \mapsto z^{k} .
\end{aligned}
$$

onde $k \in \mathbb{Z}$. Denotando por $\overline{\mathbb{B}}_{1}$ a bola fechada unitária centrada na origem $0 \in \mathbb{R}^{n+1}$, as equivalências homotópicas $\rho: \overline{\mathbb{B}}_{1} \backslash\{0\} \rightarrow \mathbb{S}^{1}$ e $j: \mathbb{S}^{1} \rightarrow \overline{\mathbb{B}}_{1} \backslash\{0\}$ são dadas por

$$
\rho(x)=\frac{x}{\|x\|} \text { e } j(y)=y
$$

respectivamente, assim, $\rho \circ f \circ j: \mathbb{S}^{1} \rightarrow \mathbb{S}^{1}$ é a restrição de $f$ a $\mathbb{S}^{1}$. Do Exemplo 3.1.3 temos $\operatorname{gr}(\rho \circ f \circ j)=k$, portanto, $\gamma_{0}(f)=k$.

A seguir apresentaremos algumas propriedades e exemplos, que além de nos ajudarem a compreender o conceito de grau local, serão utilizados mais adiante.

Teorema 3.2.6. Sejam $U$ e $V$ subconjuntos abertos de $\mathbb{R}^{n}$. Consideremos três pontos $a$, $b$ e $c$ de $\mathbb{R}^{n}$, tais que os pontos $a$ e $b$ estejam contidos em $U$ e $V$, respectivamente. Consideremos ainda $f: U \backslash\{a\} \rightarrow \mathbb{R}^{n} \backslash\{b\}$ e $g: V \backslash\{b\} \rightarrow \mathbb{R}^{n} \backslash\{c\}$ aplicações contínuas, com $f(U \backslash\{a\}) \subset V \backslash\{b\}$. Então, $\gamma_{a}(g \circ f)=\gamma_{b}(g) \gamma_{a}(f)$.

Demonstração: Tomemos $\overline{\mathbb{B}}_{\varepsilon_{1}}=\overline{\mathbb{B}}_{\varepsilon_{1}}(b) \subset V$ a bola fechada de centro $b$ e raio $\varepsilon_{1}$ e, $\overline{\mathbb{B}}_{\varepsilon}=\mathbb{B}_{\varepsilon}(a) \subset U$ a bola fechada de centro $a$ e raio $\varepsilon$ tais que $f\left(\overline{\mathbb{B}}_{\varepsilon}\right) \subset \overline{\mathbb{B}}_{\varepsilon_{1}}$. Então,

$$
\gamma_{a}(g \circ f)=g r(\rho \circ g \circ f \circ j)
$$

Sejam

$$
\rho^{1}: \overline{\mathbb{B}}_{\varepsilon_{1}} \backslash\{b\} \rightarrow \mathbb{S}^{n-1} \text { e } j^{1}: \mathbb{S}^{n-1} \rightarrow \overline{\mathbb{B}}_{\varepsilon_{1}} \backslash\{b\}
$$

as equivalências homotópicas naturais, assim, $j^{1} \circ \rho^{1} \simeq i d: \overline{\mathbb{B}}_{\varepsilon_{1}} \backslash\{b\} \rightarrow \overline{\mathbb{B}}_{\varepsilon_{1}} \backslash\{b\}$, e temos

$$
\rho \circ g \circ f \circ j \simeq \rho \circ g \circ j^{1} \circ \rho^{1} \circ f \circ j .
$$


Logo, pelo Teorema 3.1 .16

$$
\operatorname{gr}(\rho \circ g \circ f \circ j)=\operatorname{gr}\left(\rho \circ g \circ j^{1}\right) \operatorname{gr}\left(\rho^{1} \circ f \circ j\right) .
$$

Mas, $\gamma_{b}(g)=\operatorname{gr}\left(\rho \circ g \circ j^{1}\right)$, enquanto $\gamma_{a}(f)=\operatorname{gr}\left(\rho^{1} \circ f \circ j\right)$, onde $\rho^{2}: \mathbb{R}^{n} \backslash\{b\} \rightarrow \mathbb{S}^{n-1}$. Porém $\rho^{1}=\left.\rho^{2}\right|_{\overline{\mathbb{B}}_{\varepsilon_{1}} \backslash\{b\}}$, assim

$$
\rho^{1} \circ f \circ j=\rho^{2} \circ f \circ j
$$

e dai $\gamma_{a}(f)=g r\left(\rho^{1} \circ f \circ j\right)$. Portanto $\gamma_{a}(g \circ f)=\gamma_{b}(g) \gamma_{a}(f)$.

Corolário 3.2.7. Sejam $U, V$ e $W$ subconjuntos abertos de $\mathbb{R}^{n}$, e os pontos $a \in W \subset U$, $b \in V$. Consideremos $f: U \backslash\{a\} \rightarrow \mathbb{R}^{n} \backslash\{b\}$ uma aplicação contínua. Se $f$ aplica $W \backslash\{a\}$ homeomorficamente sobre $V \backslash\{b\}$, então $\gamma_{a}(f)= \pm 1$.

Demonstração: Seja a aplicação contínua $\tilde{f}=\left.f\right|_{W \backslash\{a\}}$ e $\tilde{f}^{-1}$ a sua inversa, então, do Teorema 3.2.6, temos $\gamma_{a}(I d)=\gamma_{a}\left(\tilde{f}^{-1} \circ \tilde{f}\right)=\gamma_{a}(\tilde{f}) \gamma_{b}\left(\tilde{f}^{-1}\right)$. Portanto, concluímos que $\gamma_{a}(f)= \pm 1$

Exemplo 3.2.8. Seja $f: \mathbb{R}^{n} \rightarrow \mathbb{R}^{n}$ uma aplicação linear invertível, então

$$
f\left(\mathbb{R}^{n} \backslash\{0\}\right)=\mathbb{R}^{n} \backslash\{0\} .
$$

Mostraremos que $\gamma_{0}(f)=1$ se $\operatorname{det}(f)>0$ e $\gamma_{0}(f)=-1$ se $\operatorname{det}(f)<0$.

De fato, se $\operatorname{det}(f)>0$, então $f$ pertence à componente conexa da identidade em $G l(n, \mathbb{R})$ e então existe uma aplicação contínua

$$
\begin{aligned}
\alpha:[0,1] & \rightarrow G l(n, \mathbb{R}) \\
t & \mapsto f_{t},
\end{aligned}
$$

com $f_{0}=f$ e $f_{1}=i d: \mathbb{R}^{n} \rightarrow \mathbb{R}^{n}$. Cada aplicação linear $f_{t}$ é invertível e tal que $f_{t}\left(\mathbb{R}^{n} \backslash\{0\}\right) \subset \mathbb{R}^{n} \backslash\{0\}$, assim, considerando a aplicação contínua

$$
\begin{aligned}
F: \mathbb{R}^{n} \times[0,1] & \rightarrow \mathbb{R}^{n} \\
(x, t) & \mapsto f_{t}(x),
\end{aligned}
$$


obtemos $f \simeq i d: \mathbb{R}^{n} \rightarrow \mathbb{R}^{n}$. Da Observação 3.2.4, segue que $\gamma_{0}(f)=1$.

Por outro lado, se $\operatorname{det}(f)<0$, seja

$$
\lambda: \mathbb{R}^{n} \backslash\{0\} \rightarrow \mathbb{R}^{n} \backslash\{0\}
$$

a reflexão relativamente ao hiperplano $x_{n}=0$, isto é, $\lambda\left(x_{1}, \ldots, x_{n}\right)=\left(x_{1}, \ldots, x_{n-1},-x_{n}\right)$. Segue do Exemplo 3.1 .4 que $\gamma_{0}(\lambda)=-1$ e, como $\operatorname{det}(\lambda f)>0$, temos

$$
1=\gamma_{0}(\lambda f)=\gamma_{0}(\lambda) \gamma_{0}(f)=-\gamma_{0}(f)
$$

e portanto $\gamma_{0}(f)=-1$.

Do Corolário 3.2.7 e do Exemplo 3.2.8, obtemos a seguinte proposição.

Proposição 3.2.9. Sejam $f: U \rightarrow \mathbb{R}^{n}$ uma aplicação diferenciável, um ponto $a \in U \subset \mathbb{R}^{n}$ e $b=f(a)$. Suponhamos que $f(U \backslash\{a\}) \subset \mathbb{R}^{n} \backslash\{b\}$ e admitamos ainda que a aplicação linear $d f_{a}: \mathbb{R}^{n} \rightarrow \mathbb{R}^{n}$ seja invertível. Então, $\gamma_{a}(f)=1$ se o determinante jacobiano $\operatorname{det}\left(d f_{a}\right)>0$ e $\gamma_{a}(f)=-1$ se $\operatorname{det}\left(d f_{a}\right)<0$.

Demonstração: Pelo Teorema da Função Inversa, $f$ aplica homeomorficamente uma vizinhança do ponto a sobre uma vizinhança de $b$, pelo Corolário 3.2.7, concluímos que $\gamma_{a}(f)= \pm 1$.

Considerando o Exemplo 3.2.8, basta mostrarmos que $\gamma_{a}(f)=\gamma_{0}\left(d f_{a}\right)$, onde $d f_{a}$ : $\mathbb{R}^{n} \backslash\{0\} \rightarrow \mathbb{R}^{n} \backslash\{0\}$

Sem perda de generalidade, podemos supor $a=0$ e $f(a)=0$, seja

$$
i=\inf \left\{\left\|d f_{a}(u)\right\| ; \quad u \in \mathbb{R}^{n}, \quad\|u\|=1\right\}
$$

mostremos que $i>0$.

De fato, suponhamos que $i=0$, $\operatorname{logo}$ dado $\varepsilon=\frac{1}{n}>0$ existiria $u_{n} \in \mathbb{R}^{n} \backslash\{0\}$ com $\left\|u_{n}\right\|=1$ e $\left\|d f_{a}\left(u_{n}\right)\right\|<\frac{1}{n}$. Assim, teríamos a sequência $\left\{\left\|d f_{a}\left(u_{n}\right)\right\|\right\}$ convergindo para 0 , e consequentemente a sequência $\left\{d f_{a}\left(u_{n}\right)\right\}$ também convergiria para 0. Do fato de $f$ ser de classe $C^{1}$ teríamos $u_{n} \rightarrow 0$, o que é absurdo, pois $\left\|u_{n}\right\|=1$ para todo $n \in \mathbb{N}$, logo $i>0$. 
Além disso, sabemos que numa vizinhança do 0 podemos escrever

$$
f(x)=d f_{a}(x)+\theta(x), \text { onde } \lim _{x \rightarrow 0} \frac{\|\theta(x)\|}{\|x\|}=0 .
$$

Seja $W$ aberto, com $0 \in W \subset U$ e tal que

$$
\frac{\|\theta(x)\|}{\|x\|}<i, \text { para todo } x \in W, \quad x \neq 0
$$

Então, quaisquer que sejam $x \in W \backslash\{0\}$ e $0 \leq t \leq 1$, temos

$$
\begin{aligned}
(1-t) f(x)+t d f_{a}(x) & =(1-t) d f_{a}(x)+(1-t) \theta(x)+t d f_{a}(x) \\
& =d f_{a}(x)+(1-t) \theta(x) \\
& =\|x\|\left[d f_{a}\left(\frac{x}{\|x\|}\right)+(1-t) \frac{\theta(x)}{\|x\|}\right] \neq 0 .
\end{aligned}
$$

Assim, podemos definir uma homotopia $F:(W \backslash\{0\}) \times I \rightarrow \mathbb{R}^{n} \backslash\{0\}$ entre as restrições $f, d f_{a}: W \backslash\{0\} \rightarrow \mathbb{R}^{n} \backslash\{0\}$, pondo

$$
F(x, t)=(1-t) f(x)+t d f_{a}(x)
$$

Segue da Observação 3.2 .4 que $\gamma_{0}(f)=\gamma_{0}\left(d f_{a}\right)= \pm 1$, conforme $\operatorname{det}(f)$ é positivo ou negativo.

Teorema 3.2.10. Sejam $U \subset \mathbb{R}^{n}$ um aberto, $a \in U$ e $f: U \backslash\{a\} \rightarrow \mathbb{R}^{n} \backslash\{b\}$ uma aplicação contínua, onde $b=f(a)$. Então $\gamma_{a}(f)=0$ se, e somente se, para toda bola fechada $\overline{\mathbb{B}}_{\varepsilon}(a) \subset U$ de centro $a$ e raio $\varepsilon$, existe uma aplicação contínua $g: U \rightarrow \mathbb{R}^{n} \backslash\{b\}$ que coincide com $f$ em $U \backslash \mathbb{B}_{\varepsilon}(a)$.

Demonstração: Se existe $g: U \rightarrow \mathbb{R}^{n} \backslash\{b\}$ contínua, com $g(x)=f(x)$ para todo $x \in U \backslash \mathbb{B}_{\varepsilon}(a)$, consideremos a aplicação $j=j_{a, \varepsilon}: \mathbb{S}^{n-1} \rightarrow \overline{\mathbb{B}}_{\varepsilon}(a) \backslash\{a\}$, como definida anterirmente, e chamemos de $\overline{\mathbb{B}}_{1}=\overline{\mathbb{B}}_{1}(0)$ a bola fechada unitária com centro na origem $0 \in \mathbb{R}^{n}$. 
A mesma fórmula que define $j$, define também um homeomorfismo $\tilde{j}: \overline{\mathbb{B}}_{1} \rightarrow \overline{\mathbb{B}}_{\varepsilon}(a)$, assim se considerarmos $i: \mathbb{S}^{n-1} \rightarrow \overline{\mathbb{B}}_{1}$ a inclusão, temos

$$
f \circ j=i \circ \tilde{j} \circ g: \mathbb{S}^{n-1} \rightarrow \mathbb{R}^{n} \backslash\{b\}
$$

pois $f$ coincide com $g$ em $j\left(\mathbb{S}^{n-1}\right)$. Isto significa que

$$
h=\rho \circ f \circ j: \mathbb{S}^{n-1} \rightarrow \mathbb{S}^{n-1}
$$

estende-se a uma aplicação contínua $\tilde{h}=\rho \circ g \circ \tilde{j}: \overline{\mathbb{B}}_{1} \rightarrow \mathbb{S}^{n-1}$, então, pelo Exemplo 3.1.22, $\gamma_{a}(f)=\operatorname{gr}(\rho \circ f \circ j)=0$.

Suponhamos agora que $\gamma_{a}(f)=0$ e tomemos uma bola fechada qualquer $\overline{\mathbb{B}}_{\varepsilon}(a) \subset U$, temos $g r(\rho \circ f \circ j)=0, \operatorname{com} j=j_{a, \varepsilon}: \mathbb{S}^{n-1} \rightarrow \overline{\mathbb{B}}_{\varepsilon}(a) \backslash\{a\}$, pelo Teorema 3.1.23, concluímos que

$$
\rho \circ f \circ j \simeq \text { constante. }
$$

Considerando a equivalência homotópica $j^{1}: \mathbb{S}^{n-1} \rightarrow \mathbb{R}^{n} \backslash\{b\}$, inversa de $\rho$, temos

$$
j^{1} \circ \rho \simeq i d: \mathbb{R}^{n} \backslash\{b\} \rightarrow \mathbb{R}^{n} \backslash\{b\}
$$

Segue das Equações 3.2.1 e 3.2.2 que

$$
f \circ j=i d \circ(f \circ j) \simeq j^{1} \circ \rho \circ f \circ j \simeq j^{1} \circ \text { constante }=\text { constante }: \mathbb{S}^{n-1} \rightarrow \mathbb{R}^{n} \backslash\{b\} .
$$

Na Proposição 1.2 .5 , vimos que

$$
f \circ j: \mathbb{S}^{n-1} \rightarrow \mathbb{R}^{n} \backslash\{b\}
$$

admite uma extensão contínua $k: \overline{\mathbb{B}}_{1} \rightarrow \mathbb{R}^{n} \backslash\{b\}$. Considerando novamente o homeomorfismo $\tilde{j}: \overline{\mathbb{B}}_{1} \rightarrow \overline{\mathbb{B}}_{\varepsilon}(a)$, definimos a aplicação $g: U \rightarrow \mathbb{R}^{n} \backslash\{b\}$ da seguinte forma

$$
g(x)=\left\{\begin{array}{l}
f(x), \text { se } x \in U \backslash \mathbb{B}_{\varepsilon}(a) \\
k\left(\tilde{j}^{-1}(x)\right), \text { se } x \in \overline{\mathbb{B}}_{\varepsilon}(a)
\end{array}\right.
$$


Portanto, $g$ é contínua, como queríamos demonstrar. 



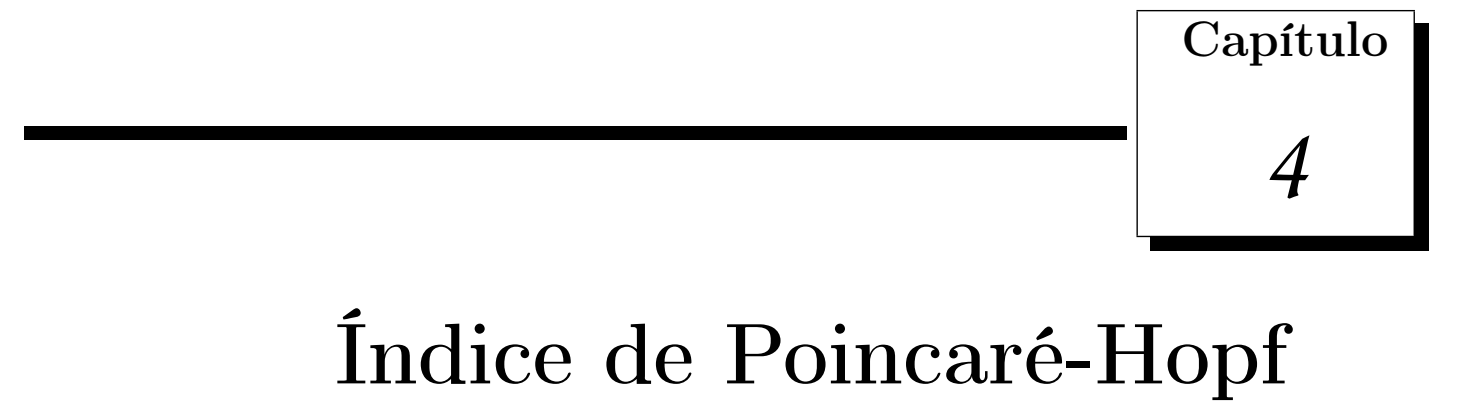

Por volta de 1885, H. Poincaré provou um importante teorema, no qual associou a cada singularidade isolada de um campo de vetores $v$ sobre uma superfície compacta e sem bordo $M^{2}$ um número, que é chamado indice da singularidade. Por exemplo, considerando a esfera $\mathbb{S}^{2}$, o teorema provado por $\mathrm{H}$. Poincaré garante que todo campo vetorial contínuo admite pelo menos uma singularidade.

O surpreendente resultado, provado por H. Poincaré, diz que a soma destes índices das singularidades de um campo contínuo $v$ tangente à uma superfície compacta e sem bordo $M^{2}$ não depende do campo $v$. Esta soma de índices é um número, chamado a característica de Euler da superfície $M^{2}$, denotado por $\chi\left(M^{2}\right)$.

Mais tarde, por volta de 1927, H. Hopf demonstrou que o teorema provado por $\mathrm{H}$. Poincaré para superfícies compactas vale mais geralmente, isto é, H. Hopf provou que dada uma variedade $m$-dimensional, compacta e sem bordo $M$ a soma dos índices das singularidades de um campo contínuo $v$ tangente à $M$ não depende do campo $v$. Este resultado passou então a ser conhecido como Teorema de Poincaré-Hopf.

Neste capítulo, apresentaremos o conceito de campos vetoriais sobre variedades diferenciáveis e de índice de singularidades isoladas de campos vetoriais definidos sobre tais objetos, demonstraremos algumas propriedades deste índice e por fim provaremos o Teorema de Poincaré-Hopf. 


\subsection{Campos de vetores}

Campos de vetores são amplamente estudados, devido a sua importância em vários ramos da matemática e também por suas várias aplicações em outras áreas como física, biologia, economia e etc. Neste capítulo, apresentaremos a definição de campo de vetores tangente a uma variedade diferenciável $M$.

Definição 4.1.1. Um campo vetorial $v$, sobre uma variedade diferenciável $M$, é uma correspondencia $v: M \rightarrow T M$ que associa a cada ponto $p \in M$ um ponto $\left(p, v_{p}\right)$ do fibrado tangente $T M$, onde $v_{p} \in T_{p} M$.

Dado um campo vetorial $v$ sobre $M$ e $\varphi: V \stackrel{a b}{\subset} M \rightarrow U \stackrel{a b}{\subset} \mathbb{R}^{m}$ uma carta de $M$, para todo $p \in V$ podemos escrever

$$
v_{p}=\sum_{i=1}^{m} \alpha_{i}(p) \frac{\partial}{\partial x_{i}}(p),
$$

onde

$$
\left\{\frac{\partial}{\partial x_{1}}(p), \ldots, \frac{\partial}{\partial x_{m}}(p)\right\}
$$

denota a base

$$
\left\{d \varphi_{\varphi(p)}^{-1}\left(e_{1}\right), \ldots, d \varphi_{\varphi(p)}^{-1}\left(e_{m}\right)\right\}
$$

do espaço vetorial tangente $T_{p} M$ associada a carta $\varphi$ em cada ponto $p=\varphi^{-1}\left(x_{1}, \ldots, x_{m}\right)$. Assim, para cada carta $\varphi$, o campo $v$ fica definido pelas $m$ funções reais $\alpha_{i}: V \rightarrow \mathbb{R}$.

Diremos que o campo $v$ é contínuo, diferenciável ou de classe $C^{k}$, quando para toda carta $\varphi$, as funções $\alpha_{i}$ forem contínuas, diferenciáveis ou de classe $C^{k}$, respectivamente.

Exemplo 4.1.2. Os campos de vetores definidos sobre um aberto $U$ de $\mathbb{R}^{2}$, que apresentamos na Definição 2.0.12, são campos de vetores tangentes a $U$, pois para todo $p \in U$ podemos identificar o espaço vetorial $T_{p} U$ com $\mathbb{R}^{2}$, para maiores detalhes veja [13] ou [17].

Exemplo 4.1.3. A aplicação definida por $v(x, y)=(-y, x)$, para todo $(x, y) \in \mathbb{S}^{1}$, é um campo de vetores tangente a $\mathbb{S}^{1}$, uma vez que em cada ponto $(x, y) \in \mathbb{S}^{1}$ o espaço tangente $T_{(x, y)} \mathbb{S}^{1}$ é o conjunto dos pontos $\left\{(u, v) \in \mathbb{R}^{2}:\langle(u, v),(x, y)\rangle=0\right\}$. 


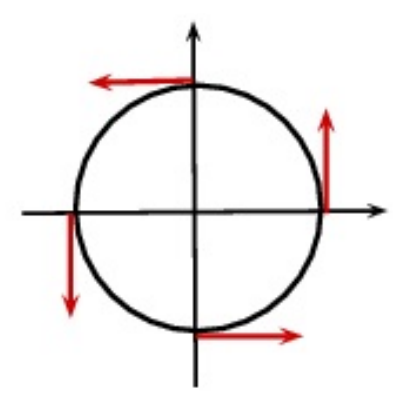

Figura 4.1: Campo de vetores sobre $\mathbb{S}^{1}$

Observação 4.1.4. Seja $M$ uma variedade diferenciável, então existe uma projeção natural $\pi: T M \rightarrow M$ definida por $\pi(p, v)=p$. Assim, segue diretamente da definição, que um campo vetorial na variedade $M$ é uma aplicação $v: M \rightarrow T M$ satisfazendo $\pi(v(p))=p$, para todo $p \in M$.

Teorema 4.1.5. Sejam $M$ uma variedade diferenciável e $T M$ o seu fibrado tangente. Todo campo vetorial diferenciável $v: M \rightarrow T M$ é um mergulho.

Demonstração: Seja $v: M \rightarrow T M$ um campo vetorial diferenciável na variedade $M$, como $\pi \circ v=I d: M \rightarrow M$ segue que $v$ é uma imersão de $M$ em $T M$.

Dessa forma, para concluírmos a demonstração só falta mostrarmos que $v$ é um homeomorfismo sobre sua imagem. Segue da definição de campo de vetores que $v: M \rightarrow T M$ é injetora, $\log 0 v$ é bijetora sobre sua imagem.

Podemos definir sobre $v(M)$ a aplicação $\left.v^{-1}\right|_{v(M)}=\left.\pi\right|_{v(M)}$, que é contínua. Portanto, $v$ é um mergulho.

Segue diretamente do Teorema 4.1.5 que, dado um campo vetorial diferenciável $v$ : $M \rightarrow T M$ sua imagem $v(M)$ é uma subvariedade de $T M$.

Definição 4.1.6. Sejam $M$ uma variedade diferenciável e $v$ um campo de vetores tangentes sobre $M$, uma singularidade do campo $v$ é um ponto $p \in M$, onde o campo $v$ se anula, ou seja, $v(p)=\overrightarrow{0}$.

Se $p \in M$ é uma singularidade do campo $v$ e se existe uma vizinhança $V$ de $M$ tal que $p$ é a única singularidade de $v$ em $V$, então $p$ é chamada uma singularidade isolada do campo $v$. 
Exemplo 4.1.7. Consideremos $v: \mathbb{R}^{3} \rightarrow \mathbb{R}^{3}$ a aplicação definida por $v(x, y, z)=$ $(-y, x, 0)$, para todo $(x, y, z) \in \mathbb{S}^{2}$. Temos que $v$ é um campo de vetores tangente a $\mathbb{S}^{2}$ (ver Figura 4.2 , e observemos que, os pontos $(0,0,-1)$ e $(0,0,1)$ são as únicas singularidades do campo $v$, além disso, é fácil ver que $(0,0,-1)$ e $(0,0,1)$ são singularidades isoladas de $v$.

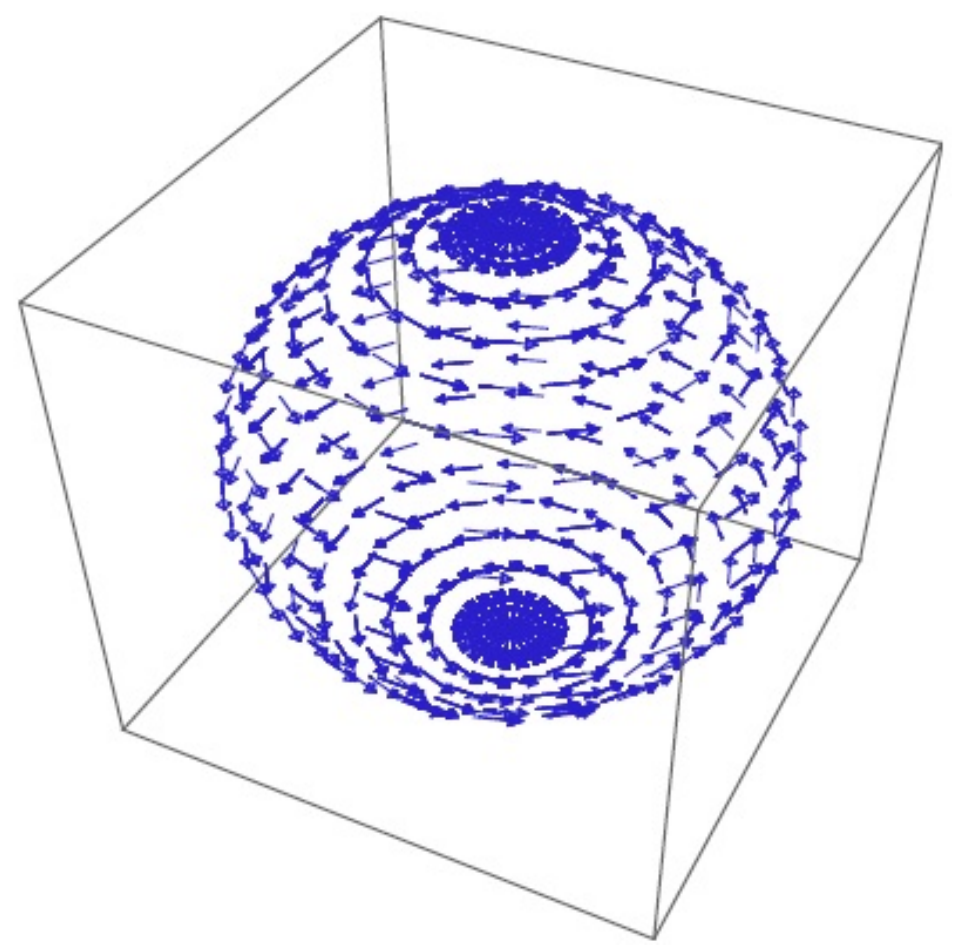

Figura 4.2: Campo de vetores sobre $\mathbb{S}^{2}$

A seguir daremos a noção de singularidade simples de um campo vetorial diferenciável definido sobre uma variedade diferenciável $M$. Veremos na seção 4.4 que este conceito está relacionado com a característica de Euler de $M$.

Definição 4.1.8. Sejam $M$ uma variedade diferenciável, $T M$ seu fibrado tangente e $v: M \rightarrow T M$ um campo de vetores diferenciável sobre $M$. Diremos que uma singularidade $p$ do campo $v$ é simples se

$$
d \phi_{p}\left(T_{p} M\right) \oplus d v_{p}\left(T_{p} M\right)=T_{(p, 0)}(T M)
$$

onde $\phi: M \rightarrow T M$ é o campo vetorial nulo, isto é, $\phi(p)=(p, 0)$ para todo $p \in M$. 
Se $p \in M$ é uma singularidade simples do campo $v: M \rightarrow T M$, geometricamente, significa que as subvariedades $\phi(M)$ e $v(M)$ interceptam-se transversalmente no ponto $(p, 0)=v(p)=\phi(p)$. Como ilustração vejamos a Figura 4.3 , onde o ponto $p_{1}$ é uma singularidades simples do campo $v$ e o ponto $p_{2}$ é uma singularidade do campo $v$, porém não é simples.

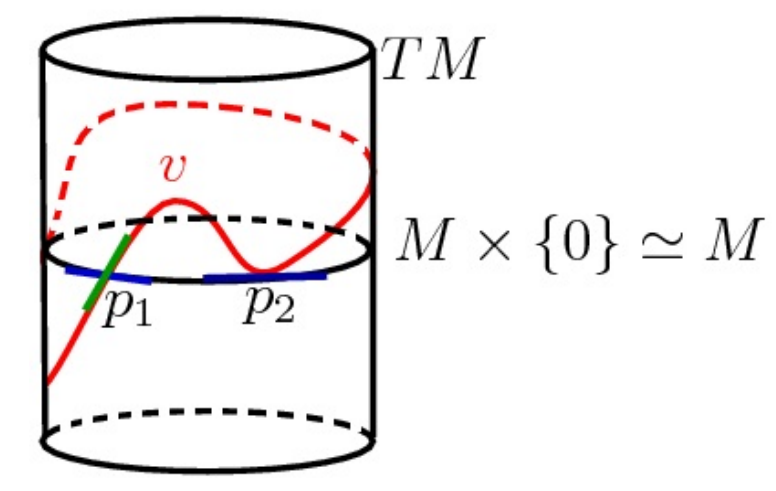

Figura 4.3: Representação geométrica de uma singularidade simples

Teorema 4.1.9. Sejam $M$ uma variedade diferenciável e $\varphi: V \rightarrow U$ uma carta de $M$, onde $V$ é um aberto de $M$ e $U$ é um aberto de $\mathbb{R}^{m}$. Dado um campo de vetores diferenciável $v: M \rightarrow T M$, como descrito abaixo

$$
v_{q}=\sum_{i=1}^{m} \alpha_{i}(q) \frac{\partial}{\partial x_{i}}(q), \quad \text { para todo } q \in V
$$

Uma singularidade $p$ de $v$ é simples se, e somente se, $\operatorname{det}([J \alpha(p)]) \neq 0$, onde $\alpha: V \rightarrow \mathbb{R}^{m}$ é a aplicação diferenciável dada por $\alpha(q)=\left(\alpha_{1}(q), \ldots, \alpha_{m}(q)\right)$.

Demonstração: Sejam $\varphi: V \rightarrow U$ uma carta de $M$, onde $p \in V$ e $U$ é um subconjunto aberto de $\mathbb{R}^{m}$, e $\tilde{\varphi}: T_{V} M \rightarrow U \times \mathbb{R}^{m}$ uma carta de $T M$, definida por

$$
\tilde{\varphi}\left(x_{1}, \ldots, x_{m}, v_{1}, \ldots, v_{m}\right)=(p, u)
$$

onde $T_{V} M=\bigcup_{p \in V}\{p\} \times T_{p} M, p=\varphi^{-1}\left(x_{1}, \ldots, x_{m}\right)$ e $u$ é o vetor tangente a $M$ em $p$ cujas coordenadas locais com respeito a $\varphi$ são $\left(v_{1}, \ldots, v_{m}\right)$. 
Em termos das cartas $\varphi$ em $M$ e $\tilde{\varphi}$ em $T M$, o campo $v$ e o campo vetorial nulo $\phi: M \rightarrow T M$ se exprimem da forma

$$
\begin{aligned}
& \phi:\left(x_{1}, \ldots, x_{m}\right) \rightarrow\left(x_{1}, \ldots, x_{m}, 0, \ldots, 0\right) \\
& v:\left(x_{1}, \ldots, x_{m}\right) \rightarrow\left(x_{1}, \ldots, x_{m}, \alpha_{1}\left(x_{1}, \ldots, x_{m}\right), \ldots, \alpha_{m}\left(x_{1}, \ldots, x_{m}\right)\right) .
\end{aligned}
$$

Seja a base $\left\{\frac{\partial}{\partial x_{1}}(p), \ldots, \frac{\partial}{\partial x_{m}}(p)\right\}$ de $T_{p} M$, associada a carta $\varphi$, então os vetores

$$
\begin{aligned}
& d \phi_{p}\left(\frac{\partial}{\partial x_{1}}(p)\right), \ldots, d \phi_{p}\left(\frac{\partial}{\partial x_{m}}(p)\right) \\
& d v_{p}\left(\frac{\partial}{\partial x_{1}}(p)\right), \ldots, d v_{p}\left(\frac{\partial}{\partial x_{m}}(p)\right)
\end{aligned}
$$

são bases de $d \phi_{p}\left(T_{p} M\right)$ e de $d v_{p}\left(T_{p} M\right)$, respectivamente, do fato de $p$ ser uma singularidade simples de $v$, segue que

$$
d \phi_{p}\left(\frac{\partial}{\partial x_{1}}(p)\right), \ldots, d \phi_{p}\left(\frac{\partial}{\partial x_{m}}(p)\right), d v_{p}\left(\frac{\partial}{\partial x_{1}}(p)\right), \ldots, d v_{p}\left(\frac{\partial}{\partial x_{m}}(p)\right)
$$

é uma base do espaço vetorial $T_{(p, 0)}(T M)$.

Em relação a carta $\tilde{\varphi}$, cada vetor $d \phi_{p}\left(\frac{\partial}{\partial x_{i}}(p)\right)$ tem coordenadas $(0, \ldots, 1,0, \ldots, 0)$, isto é, todas as coordenadas são nulas, exceto a $i$-ésima que é igual a 1 , as coordenadas do vetor $d v_{p}\left(\frac{\partial}{\partial x_{i}}(p)\right)$, em relação a mesma parametrização $\tilde{\varphi}$ são

$$
\left(0, \ldots, 1,0, \ldots, \frac{\partial \alpha_{1}}{\partial x_{i}}(p), \ldots, \frac{\partial \alpha_{m}}{\partial x_{i}}(p)\right)
$$

onde as $m$ primeiras coordenadas são nulas, com exceção da $i$-ésima. Assim, a matriz quadrada cujas colunas são as coordenadas dos vetores $d \phi_{p}\left(\frac{\partial}{\partial x_{i}}(p)\right)$ e $d v_{p}\left(\frac{\partial}{\partial x_{i}}(p)\right)$, relativamente a parametrização $\tilde{\varphi}$, assume a forma abaixo, onde os quatro blocos são matrizes $m \times m$

$$
A=\left[\begin{array}{cc}
I & I \\
0 & {[J \alpha(p)]}
\end{array}\right] .
$$

Os $2 m$ vetores em questão são linearmente independentes se, e somente se, $\operatorname{det}(M) \neq 0$. Mas, $\operatorname{det}(M)=\operatorname{det}([J \alpha(p)])$. Portanto, $\operatorname{det}([J \alpha(p)]) \neq 0$. 
Deste resultado obtemos o seguinte corolário.

Corolário 4.1.10. Sejam $M$ uma variedade diferenciável e $T M$ seu fibrado tangente, as singularidades simples de um campo vetorial diferenciável $v: M \rightarrow T M$ são isoladas no conjunto de todas as singularidades de $v$.

Demonstração: Sejam $p \in M$ uma singularidade simples de $v, \varphi: V \rightarrow U$ uma carta de $M$, onde $V$ é um aberto de $M$ contendo $p$ e $U$ é um aberto de $\mathbb{R}^{m}$, podemos descrever $v_{q}$ da seguinte forma

$$
v_{q}=\sum_{i=1}^{m} \alpha_{i}(q) \frac{\partial}{\partial x_{i}}(q)
$$

para todo $q \in V$.

Consideremos a aplicação diferenciável $\alpha: V \rightarrow \mathbb{R}^{m}$ definida por

$$
\alpha(q)=\left(\alpha_{1}(q), \ldots, \alpha_{m}(q)\right)
$$

do Teorema 4.1.9, segue que $\operatorname{det}([J \alpha(p)]) \neq 0$, logo, pelo Teorema da Função Inversa, existe uma vizinhança $W$ de $p$ em $M$, tal que a aplicação $\alpha$ é um difeomorfismo de $W$ sobre uma vizinhança de $(0, \ldots, 0) \in \mathbb{R}^{m}$, portanto, para todo $q \neq p$ de $W$, temos $\left(\alpha_{1}(q), \ldots, \alpha_{m}(q)\right) \neq(0, \ldots, 0)$, isto é, nenhum outro ponto $q \in W$, além de $p$, é uma singularidade de $v$.

Observação 4.1.11. Sejam $M$ uma variedade diferenciável compacta, $v: M \rightarrow T M$ um campo de vetores contínuo e $\phi: M \rightarrow T M$ o campo de vetores nulo, então, $\phi(M)$ é um subconjunto compacto de TM, mas TM é um espaço topológico Hausdorff, logo $\phi(M)$ é um subconjunto fechado de TM. Do fato de $v$ ser continuo e $M$ ser compacta, temos que $v^{-1}(\phi(M))$ é um subconjunto compacto de $M$, mas, $v^{-1}(\phi(M))$ é exatamente o conjunto dos pontos singulares de $v$. Segue então do Corolário 4.1 .10 que se $M$ é uma variedade diferenciável compacta e vé um campo diferenciável sobre $M$ cujas singularidades são todas simples, estas são em número finito apenas.

A seguir, mostraremos que todo campo diferenciável $v: M \rightarrow T M$ pode ser aproximado por um campo de vetores diferenciável $w: M \rightarrow T M$, cujas singularidades são todas simples. 
No entanto, para demonstrar esse resultado, precisaremos dar uma estrutura topológica para o conjunto $\mathcal{F}(M, N)$ das aplicações diferenciáveis da variedade $M$ na variedade $N$, para isto, apresentamos as seguintes definições.

Definição 4.1.12. Sejam $M$ e $N$ variedades diferenciáveis, dadas $f, g: M \rightarrow N$ aplicações diferenciáveis, definimos a distância

$$
d_{0}(f, g)=\sup \{|f(p)-g(p)| ; p \in M\}
$$

onde a norma $|f(p)-g(p)|$ é a do espaço $\mathbb{R}^{k}$ em que $N$ esta imersa.

Chamaremos de topologia $C^{0}$ a topologia sobre $\mathcal{F}(M, N)$ definida pela distância $d_{0}$.

Definição 4.1.13. Sejam $M$ e $N$ variedades diferenciáveis, dadas $f, g: M \rightarrow N$ aplicações diferenciáveis, definimos a distância

$$
d_{1}(f, g)=\max \left\{d_{0}(f, g), \quad \sup \left\{\left|d f_{p}(u)-d g_{p}(u)\right| ; u \in T_{p} M, \quad|u|=1, \quad p \in M\right\}\right\} .
$$

Como na definição anterior $\left|d f_{p}(u)-d g_{p}(u)\right|$ é a norma de $\mathbb{R}^{k}$. A topologia definida pela distância $d_{1}$ em $\mathcal{F}(M, N)$ é chamada topologia $C^{1}$.

Teorema 4.1.14. Seja $M$ uma variedade diferenciável, o conjunto de todos os difeomorfismos $f: M \rightarrow M$ é um aberto na topologia $C^{1}$.

Além das topologias introduzidas anteriormente, para demonstrar o resultado que comentamos acima, precisaremos de um resultado conhecido como "Lema da Transversalidade", o qual apresentaremos a seguir.

Teorema 4.1.15. (Lema da Transversalidade) Sejam $M, N$ variedades diferenciáveis, $f: M \rightarrow N$ uma aplicação diferenciável e $S \subset N$ uma subvariedade de $N$. Consideremos $X \subset M$ um subconjunto fechado de $M$ tal que $f$ é transversal a $S$ em todos os pontos de $X \cap f^{-1}(S)$. Então, dado $\varepsilon>0$, existe uma aplicação diferenciável $g: M \rightarrow N$ tal que $d_{1}(f, g)<\varepsilon$, além disso $g$ é transversal a $S$ e coincide com $f$ em $X$.

Demonstraremos a seguir o principal teorema dessa seção. 
Teorema 4.1.16. Sejam $M$ uma variedade diferenciável e $T M$ o seu fibrado tangente, todo campo vetorial diferenciável $v: M \rightarrow T M$ pode ser arbitrariamente aproximado, na topologia $C^{1}$, por um campo vetorial diferenciável cujas as singularidades são todas simples.

Demonstração: Consideremos o campo vetorial identicamente nulo $\phi: M \rightarrow T M$. Fazendo $S=\phi(M)$ no Teorema 4.1.15, vemos que é possível aproximar arbitrariamente, na topologia $C^{1}$, a aplicação $v: M \rightarrow T M$ por uma aplicação diferenciável $g: M \rightarrow T M$ que é transversal a $\phi(M)$, isto é, é possível aproximar $v$ por um campo diferenciável $g$, cujas singularidades são todas simples.

Observação 4.1.17. Na demonstração do Teorema 4.1.16 o subconjunto $v^{-1}(\phi(M)) \subset M$ desempenha o papel do subconjunto X considerado nas hipóteses do Lema da Transversalidade.

\subsection{O índice de Poincaré-Hopf}

No capítulo anterior, definimos o grau local $\gamma_{a}(f)$ da aplicação $f$ no ponto $a \in U$. Nesta seção, utilizaremos o grau local de uma aplicação em um dado ponto, para definirmos o índice de Poincaré-Hopf de uma singularidade isolada de um campo de vetores contínuo $v$ sobre uma variedade diferenciável $M$.

Uma definição equivalente deste índice, porém com outras ferramentas, é dada em [5].

Sejam $v$ um campo vetorial contínuo sobre uma variedade diferenciável $m$-dimensional $M, p \in M$ uma singularidade isolada de $v$ e $V$ uma vizinhança de $p$ tal que $v_{p}=0$, mas $v_{q} \neq 0$ para todo $q \in V \backslash\{p\}$.

Podemos supor que $V$ é domínio de uma carta $\varphi: V \rightarrow U \subset \mathbb{R}^{m}$, consideremos $U=\varphi(V) \subset \mathbb{R}^{m}$ e $a=\varphi(p) \in U$, então para todo $q \in V$, temos

$$
v_{q}=\sum_{i=1}^{m} \alpha_{i}(\varphi(q)) \frac{\partial}{\partial x_{i}}(q)
$$


onde, $\alpha_{i}: U \rightarrow \mathbb{R}$ é uma função contínua para cada $i=1, \ldots, m$, e o conjunto

$$
\left\{\frac{\partial}{\partial x_{1}}(p), \ldots, \frac{\partial}{\partial x_{m}}(p)\right\}
$$

denota a base

$$
\left\{d \varphi_{\varphi(p)}^{-1}\left(e_{1}\right), \ldots, d \varphi_{\varphi(p)}^{-1}\left(e_{m}\right)\right\}
$$

do espaço vetorial $T_{p} M$ associada a carta $\varphi: V \rightarrow U$ (que como dissemos em 1.1.27 é uma base positiva de $\left.T_{p} M\right)$. Obtemos assim uma aplicação contínua $f: U \rightarrow \mathbb{R}^{m}$, definida por

$$
f(u)=\left(\alpha_{1}(u), \ldots, \alpha_{m}(u)\right), \quad u \in U
$$

Como $p$ é a única singularidade de $v$ em $V$, temos $f(u)=0 \in \mathbb{R}^{m}$ apenas para $u=a$, onde $f(U \backslash\{a\}) \subset \mathbb{R}^{m} \backslash\{0\}$.

Definição 4.2.1. Sejam $v$ um campo vetorial contínuo sobre uma variedade diferenciável $m$-dimensional $M$ orientada, $p \in M$ uma singularidade isolada de $v$ e $\varphi: V \rightarrow U$ uma carta de $M$, onde $V$ é uma vizinhança do ponto $p$ e $U$ é um aberto de $\mathbb{R}^{m}$. Consideremos $a=\varphi(p) \in U$ e $f: U \backslash\{a\} \rightarrow \mathbb{R}^{m} \backslash\{0\}$ a aplicação contínua definida acima. Definimos o índice de Poincaré-Hopf de $v$ em $p$ como sendo o grau local $\gamma_{a}(f)$ da aplicação $f$ em $a$. Denotaremos tal índice por, $\operatorname{Ind}_{P H}(v, p)$.

Sejam $v: \mathbb{R}^{2} \rightarrow \mathbb{R}^{2}$ um campo de vetores e $p \in \mathbb{R}^{2}$ uma singularidade isolada de $v$, no Capítulo 2, definimos o índice de Poincaré-Hopf de $v$ em $p$, como sendo o número de voltas positivas menos o número de voltas negativas que a aplicação de Gauss $\gamma: \mathbb{S}_{\varepsilon} \rightarrow \mathbb{S}^{1}$ dá em torno de $\mathbb{S}^{1}$ quando $v$ percorre a circunferência $\mathbb{S}_{\varepsilon}$ de centro $p$ e raio $\varepsilon$.

Notemos que a aplicação $\rho \circ f \circ j: \mathbb{S}^{1} \rightarrow \mathbb{S}^{1}$, onde $f$ é a aplicação definida anteriormente, desempenha o mesmo papel que a aplicação de Gauss $\gamma$. Além disso, na Seção 3.2, mostramos que o grau local de uma aplicação contínua em um dado ponto não depende da bola fechada que tomamos em torno deste ponto. Então, podemos dizer que $\operatorname{gr}(\rho \circ f \circ j)=$ $\operatorname{gr}(\gamma)$.

Notemos ainda, que o número de voltas positivas (negativas) que a aplicação de Gauss $\gamma$ dá em torno de $\mathbb{S}^{1}$ quando $v$ percorre a circunferência $\mathbb{S}_{\varepsilon}$, é na verdade o número de 
pontos positivos (negativos) em $\gamma^{-1}(q)$, onde $q \in \mathbb{S}^{1}$ é um valor regular de $\gamma$. Portanto, a definição de índice de Poincaré-Hopf que apresentamos acima coincide com a definição apresentada no Capítulo 2.

Teorema 4.2.2. Sejam $v$ um campo vetorial contínuo sobre uma variedade diferenciável $m$-dimensional $M$ orientada, e $p \in M$ uma singularidade isolada de $v$. O índice de Poincaré-Hopf de $v$ em $p$ não depende da escolha da carta $\varphi$.

Demonstração: Sejam $\varphi$ e $\psi$ duas cartas, válidas em alguma vizinhança do ponto $p$. Como o grau local depende apenas de uma vizinhaça arbitrariamente pequena do ponto onde ele é tomado, podemos admitir que as cartas $\varphi$ e $\psi$ são definidas na mesma vizinhança $V$ do ponto $p \in M$ e que $U=\varphi(V)$ é uma bola de centro $a=\varphi(p)$.

Sejam $W=\psi(V), b=\psi(p)$ e

$$
v_{q}=\sum_{i=1}^{m} \beta_{i}(\psi(q)) \frac{\partial}{\partial y_{i}}(q), \quad q \in V
$$

assim, fica definida a aplicação contínua $g: W \rightarrow \mathbb{R}^{m}$, onde

$$
g(w)=\left(\beta_{1}(w), \ldots, \beta_{m}(w)\right), \quad w \in W
$$

$\operatorname{com} g(b)=0$ e $g(W \backslash\{b\}) \subset \mathbb{R}^{m} \backslash\{0\}$

Devemos mostrar que $\gamma_{a}(f)=\gamma_{b}(g)$, a fórmula de mudança de coordenadas de um vetor nos dá, para cada $q \in V$ e $i=1, \ldots, m$

$$
\beta_{i}(\psi(q))=\sum_{j=1}^{m} \frac{\partial y_{i}}{\partial x_{j}}(\varphi(q)) \alpha_{j}(\varphi(q))
$$

se, para cada ponto $u \in U$, indicarmos com $T_{u}=d\left(\psi \circ \varphi^{-1}\right)_{u}$ a aplicação linear de $\mathbb{R}^{m}$, induzida pelo difeomorfismo $\psi \circ \varphi^{-1}: U \rightarrow W$ no ponto $u \in U$, as equações acima significam que

$$
g(\psi(q))=T_{\varphi(q)}[f(\varphi(q))], \quad q \in V
$$


Podemos ainda escrever esta relação da seguinte forma

$$
\left[g \circ\left(\psi \circ \varphi^{-1}\right)\right](u)=T_{u}(f(u)), \text { para todo } u \in U \text {. }
$$

Consideremos a aplicação $S: U \backslash\{a\} \rightarrow \mathbb{R}^{m} \backslash\{0\}$, definida por $S(u)=T_{u}(f(u))$. A igualdade 4.2.1 implica

$$
\gamma_{b}(g) \gamma_{a}\left(\psi \circ \varphi^{-1}\right)=\gamma_{a}(S)
$$

Por outro lado, a aplicação $F:(U \backslash\{a\}) \times I \rightarrow \mathbb{R}^{m} \backslash\{0\}$, definida por

$$
F(u, t)=T_{(1-t) u+t a}(f(u))
$$

é uma homotopia entre as aplicações $S: U \backslash\{a\} \rightarrow \mathbb{R}^{m} \backslash\{0\}$ e $T_{a} \circ f: U \backslash\{a\} \rightarrow \mathbb{R}^{m} \backslash\{0\}$. Segue desta homotopia, que

$$
\gamma_{a}(S)=\gamma_{0}\left(T_{a}\right) \gamma_{a}(f)
$$

Mas $T_{a}=d\left(\psi \circ \varphi^{-1}\right)_{a}$ é uma aplicação linear invertível, logo,

$$
\gamma_{0}\left(T_{a}\right)=\gamma_{a}\left(y^{-1} \circ x\right)= \pm 1
$$

Assim, as igualdades 4.2.2 e 4.2.3 implicam que $\gamma_{b}(g)=\gamma_{a}(f)$.

Na seção 4.1, apresentamos o conceito de singularidade simples de um campo vetorial diferenciável $v: M \rightarrow T M$. Calculemos agora o índice de Poincaré-Hopf de uma singularidade simples de $v$.

Teorema 4.2.3. Sejam $M$ uma variedade diferenciável orientada, $v: M \rightarrow T M$ um campo vetorial diferenciável e $p \in M$ uma singularidade $\operatorname{simples~de~} v$, então $\operatorname{Ind}_{P H}(v, p)=$ \pm 1 .

Demonstração: Seja $p \in M$ uma singularidade simples do campo vetorial diferenciável $v$. Como vimos no Corolário 4.1.10 a singularidade $p$ é isolada, logo existe uma vizinhança $V$ de $p$ tal que $v_{p}=0$, mas $v_{q} \neq 0$ para todo $q \in V \backslash\{p\}$. 
Podemos supor que $V$ seja domínio de uma carta $\varphi: V \rightarrow U$, onde $U$ é um aberto de $\mathbb{R}^{m}$, consideremos $a=\varphi(p) \in U$, então para todo $q \in V$, temos

$$
v_{q}=\sum_{i=1}^{m} \alpha_{i}(\varphi(q)) \frac{\partial}{\partial x_{i}}(q)
$$

onde, para cada $i=1, \ldots, m, \alpha_{i}: U \rightarrow \mathbb{R}$ é uma função diferenciável. Obtemos assim uma aplicação diferenciável $f: U \rightarrow \mathbb{R}^{m}$, definida por

$$
f(u)=\left(\alpha_{1}(u), \ldots, \alpha_{m}(u)\right), \quad u \in U
$$

Definamos a aplicação $\alpha \circ \varphi: V \rightarrow \mathbb{R}^{m}$ colocando

$$
\alpha \circ \varphi(q)=\left(\alpha_{1} \circ \varphi(q), \ldots, \alpha_{m} \circ \varphi(q)\right)
$$

do Teorema 4.1.9, segue que $d(\alpha \circ \varphi)_{p}$ é uma aplicação linear invertível, mas $\varphi: V \rightarrow U$ é um difeomorfismo, então a derivada da aplicação $\alpha=f: U \rightarrow \mathbb{R}^{m}$ dada por $d \alpha_{a}=d f_{a}$ é também uma aplicação linear invertível.

Da Proposição 3.2 .9 temos que $\gamma_{a}(f)=1$ se o determinante jacobiano $\operatorname{det}[J f(a)]>0$, e $\gamma_{a}(f)=-1$ se o determinante jacobiano $\operatorname{det}([J f(a)])<0$. Portanto, $\operatorname{Ind}_{P H}(v, p)=1$ se $\operatorname{det}[J f(a)]>0$ e $\operatorname{Ind}_{P H}(v, p)=-1$ se $\operatorname{det}([J f(a)])<0$.

Observação 4.2.4. Sejam $M$ uma variedade diferenciável complexa e $v: M \rightarrow T M$ um campo vetorial holomorfo definido sobre $M$, neste caso, podemos provar que a recíproca do Teorema 4.2.3 é verdadeira. Mais precisamente, se $M$ é uma variedade diferenciável complexa, $v: M \rightarrow T M$ é um campo vetorial holomorfo e $p \in M$ é uma singularidade de $v$ tal que $\operatorname{Ind}_{P H}(v, p)= \pm 1$, então p é uma singularidade simples de $v$.

Sejam $M$ uma variedade diferenciável compacta e orientada, e $v$ um campo de vetores cujas singularidades são todas simples, mostraremos que a soma dos índices destas singularidades é invariante em relação a escolha do campo. Para este propósito, a seguir definiremos o importante conceito de número de interseção. 
Definição 4.2.5. Sejam $M$ uma variedade diferenciável $m$-dimensional, compacta e orientada, $N$ uma variedade diferenciável $n$-dimensional orientada e $S$ uma subvariedade $s$-dimensional e compacta de $N$, onde $s=n-m$.

Consideremos uma aplicação diferenciável $f: M \rightarrow N$ transversal à subvariedade $S$, dizemos que um ponto $p \in f^{-1}(S)$ é positivo ou negativo conforme a imagem por $d f_{p}$ de uma base positiva $\left\{e_{1}, \ldots, e_{m}\right\}$ de $T_{p} M$, seguida de uma base positiva $\left\{\tilde{e}_{1}, \ldots, \tilde{e}_{s}\right\}$ de $T_{f(p)} S$ for uma base positiva ou negativa $\left\{d f_{p}\left(e_{1}\right), \ldots, d f_{p}\left(e_{m}\right), \tilde{e}_{1}, \ldots, \tilde{e}_{s}\right\}$ de $T_{f(p)} N$.

Nas hipóteses acima, se uma aplicação diferenciável $f: M \rightarrow N$ for transversal a uma subvariedade $S$, temos que $f^{-1}(S)$ será uma subvariedade 0-dimensional fechada de $M$, então $f^{-1}(S)$ é um conjunto com uma quantidade finita de pontos. Assim, temos a seguinte definição.

Definição 4.2.6. Sejam $M$ uma variedade diferenciável $m$-dimensional, compacta e orientada, $N$ uma variedade diferenciável $n$-dimensional orientada e $S$ uma subvariedade $s$-dimensional e compacta de $N$, onde $s=n-m$.

Consideremos uma aplicação diferenciável $f: M \rightarrow N$ transversal a subvariedade $S$, definimos o número de interseção de $f(M)$ e $S$, que denotaremos por $f(M) \# S$, como o número de pontos positivos menos o número de pontos negativos em $f^{-1}(S)$.

Devido ao sinal da base $\left\{d f_{p}\left(e_{1}\right), \ldots, d f_{p}\left(e_{m}\right), \tilde{e}_{1}, \ldots, \tilde{e}_{s}\right\}$ de $T_{p} N$, a ordem de $f(M) \# S$ (primeiro $f(M)$ depois $S$ ) é importante.

Observação 4.2.7. Sejam $M, N$ variedades diferenciáveis m-dimensionais orientadas, com $M$ compacta, $f: M \rightarrow N$ uma aplicação diferenciável e um ponto $p \in N$, a aplicação $f$ é transversal à subvariedade $S=\{p\}$ se, e somente se, $p$ é um valor regular de $f$, nesse caso, $g_{p}(f)=f(M) \#\{p\}$, o que inclui a noção de grau como caso particular do conceito de número de interseção.

Sejam $v: M \rightarrow T M$ um campo vetorial diferenciável e $\phi: M \rightarrow T M$ o campo vetorial nulo, se as subvariedades $v(M)$ e $\phi(M)$ são transversais podemos definir o número de interseção da forma abaixo.

Definição 4.2.8. Sejam $M$ uma variedade diferenciável $m$-dimensional campacta e orientada, e $v: M \rightarrow T M$ um campo diferenciável, tal que $v(M)$ e $\phi(M)$ são subvariedades 
transversais de $T M$. O conjunto $v(M) \cap \phi(M)$ é finito e esta em correspondência biunívoca com o conjunto $\pi(v(M) \cap \phi(M))=\left\{p_{1}, \ldots, p_{r}\right\} \subset M$, onde $\pi: T M \rightarrow M$ é a projeção $\pi(p, u)=p$. Os pontos $p_{i}$ são precisamente os pontos de $M$ tais que $v\left(p_{i}\right)=\phi\left(p_{i}\right)=\left(p_{i}, 0\right)$. Diremos que um ponto $p_{i}$ é positivo se, dada uma base positiva $\left\{e_{1}, \ldots, e_{m}\right\}$ de $T_{p_{i}} M$ o conjunto $\left\{v_{p_{i}}\left(e_{1}\right), \ldots, v_{p_{i}}\left(e_{m}\right), \phi_{p_{i}}\left(e_{1}\right), \ldots, \phi_{p_{i}}\left(e_{m}\right)\right\}$, assim ordenado, for uma base positiva de $T_{v\left(p_{i}\right)} T M$. O número de interseção de $v(M)$ e $\phi(M)$, que denoteremos por $v(M) \# \phi(M)$, é o número de pontos positivos menos o número de pontos negativos em $\pi(v(M) \cap \phi(M))$.

Observação 4.2.9. Se as subvariedades $v(M)$ e $\phi(M)$ são transversais então todas as singularidades de $v$ são simples. Além disso, $v(M) \# \phi(M)$ é a soma dos indices das singularidades simples de $v$.

O fato principal a respeito do número de interseção, é que o mesmo é invariante por homotopia.

Teorema 4.2.10. Sejam $M$ uma variedade diferenciável $m$-dimensional, compacta e orientada, $N$ uma variedade diferenciável $n$-dimensional orientada e $S$ uma subvariedade $s$-dimensional e compacta de $N$, onde $s=n-m$.

Consideremos $f, g: M \rightarrow N$ aplicações diferenciáveis transversais a subvariedade $S$, se $f$ e $g$ são homotópicas, então $f(M) \# S=g(M) \# S$.

Utilizando o Teorema 4.2.10 podemos provar que a soma dos índices de um campo vetorial diferenciável, sobre uma variedade compacta e orientada, cujas singularidades são todas simples é um invariante de $M$.

Teorema 4.2.11. Sejam $M$ uma variedade diferenciável compacta e orientada, e $v: M \rightarrow$ $T M$ um campo de vetores diferenciável cujas singularidades são todas simples. Então, a soma dos índices das singularidades de $v$ não depende da escolha do campo $v$.

Demonstração: Seja $\phi: M \rightarrow T M$ o campo diferenciável nulo, como todas as singularidades do campo $v$ são simples, segue que as subvariedades $v(M)$ e $\phi(M)$ são transversais.

Consideremos agora, um outro campo diferenciável $w: M \rightarrow T M$ cujas singularidades também são todas simples, a aplicação $H: M \times[0,1] \rightarrow T M$ dada por

$$
H(p, t)=(1-t) v(p)+t w(p)
$$


é uma homotopia entre $v$ e $w$.

Portanto, do Teorema 4.2.10 segue que $v(M) \# \phi(M)=w(M) \# \phi(M)$.

Sejam $M$ uma variedade diferenciável compacta e $v: M \rightarrow T M$ um campo diferenciável cujas singularidades são todas simples, na Seção 4.3, mostraremos que a soma dos índices das singularidades de $v$ é igual a característica de Euler de $M$.

\subsection{Característica de Euler}

Dado um poliedro $P \subset \mathbb{R}^{3}$ de dimensão 2, L. Euler foi o primeiro a chamar a atenção para o número $V-A+F$, onde $V$ é o número de vértices de $P, A$ é o número de arestas e $F$ o número de faces.

Este número é, de fato, muito importante, e coube a H. Poincaré descobrir o significado do número que L. Euler encontrou e, a partir daí, o número $V-A+F$ se mostrou muito fértil, como parte central de notáveis igualdades e como ponto de partida para importantes generalizações. O número $\chi(P)=V-A+F$ é chamado a característica de Euler do poliedro $P$.

Nesta seção, apresentaremos uma definição mais geral deste objeto, que é uma generalização para poliedros $P$ de qualquer dimensão, ou mais geralmente, para espaços topológicos triangularizáveis.

Além de ser um conceito extremamente importante e de ser considerada como um verdadeiro traço de união entre ramos distintos da matemática, como por exemplo a Topologia e Geometria Diferencial, provaremos mais adiante, que a soma dos índices de todas as singularidades isoladas de um campo de vetores contínuo $v$, definido sobre uma variedade diferenciável compacta e sem bordo $M$, é igual a característica de Euler de $M$.

Em primeiro lugar, vejamos o que é um poliedro $n$-dimensional.

Definição 4.3.1. Um poliedro $P$ no espaço $\mathbb{R}^{n}$ é uma coleção finita de simplexos em $\mathbb{R}^{n}$ tais que:

i) Se $S$ é um simplexo de $P$, então toda face de $S$ também o é; 
ii) Se $S, T$ são simplexos de $P$, então a interseção $S \cap T$ é uma face comum a $S$ e $T$, ou é vazia.

Agora, que sabemos o que é um poliedro, podemos apresentar a seguinte definição.

Definição 4.3.2. Seja $P$ um poliedro finito $n$-dimensional e denotemos por $n_{i}$ o número de simplexos $i$-dimensionais desse poliedro. A característica de Euler de $P$ é definida por

$$
\chi(P)=\sum_{i}(-1)^{i} n_{i}
$$

A definição acima, é uma generalização do conceito de característica de Euler, para poliedros de dimensão maior ou igual a 2. Dado um espaço topológico $X$, em quais condições podemos definir a característica de Euler deste espaço $X$ ?

Com o intuito de responder esta questão, apresentaremos a seguinte definição.

Definição 4.3.3. Seja $X$ um espaço topológico, dizemos que $X$ é triangularizável se existe um homeomorfismo $h:|K| \rightarrow X$, para algum poliedro $K$ (onde, $|K|$ é o espaço topológico dado pelo conjunto $K$ com a topologia induzida do espaço ambiente). Neste caso, $(K, h)$ é dito uma triangularização de $X$.

Dado um espaço topológico $X$, nem sempre existe uma triangularização de $X$, porém, quando $X$ é uma variedade diferenciável uma condição suficiente para que $X$ admita uma triangularição é ser compacta.

Teorema 4.3.4. Seja $M$ uma variedade diferenciável compacta, então existe uma triangularização $(K, h)$ de $M$.

No caso em que $M$ é uma superfície, isto é, uma variedade diferenciável 2-dimensional, o resultado acima foi demonstrado por Whitehead em 1940 e pode ser visto em [28] (para o caso geral, veja [4] pg 307).

Dadas duas triangularizações de um mesmo espaço topológico $X$ é razoável esperarmos que as características de Euler dos poliedros associados sejam iguais. O teorema abaixo, devido a H. Poincaré, mostra exatamente isso. 
Teorema 4.3.5. Sejam $(K, h)$ e $\left(K^{\prime}, h^{\prime}\right)$ duas triangularizações do mesmo espaço $X$. Então, $\chi(K)=\chi\left(K^{\prime}\right)$.

Definição 4.3.6. Sejam $X$ um espaço topológico triangularizável e $(K, h)$ uma triangularização de $X$, onde $K$ é um poliedro homeomorfo ao espaço $X$, através do homeomorfismo $h:|K| \rightarrow X$. O número $\chi(K)$ é chamado característica de Euler de $X$ e denotado por $\chi(X)$.

Teorema 4.3.7. Sejam $X$ e $Y$ espaços topológicos triangularizáveis, se o espaço $X$ é homeomorfo ao espaço $Y$, então $\chi(X)=\chi(Y)$.

Do teorema acima, resulta que $\chi(X)$ é um invariante topológico.

Exemplo 4.3.8. Consideremos a esfera $\mathbb{S}^{2}$ e o homeomorfismo entre a esfera $\mathbb{S}^{2}$ e o cubo de arestas de comprimento 1. Consideremos a triangularização do cubo dada na Figura 4.4, então
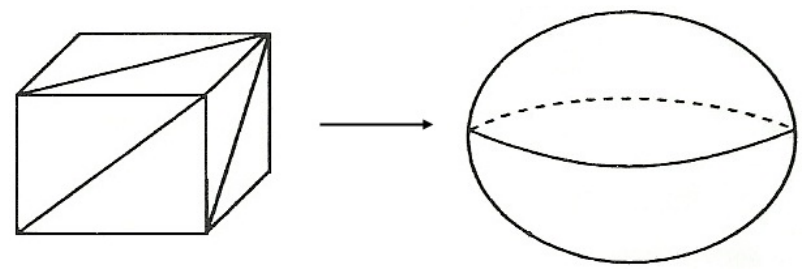

Figura 4.4: Triangularização da esfera $\mathbb{S}^{2}$

$$
\chi\left(\mathbb{S}^{2}\right)=8-18+12=2 .
$$

Exemplo 4.3.9. Consideremos o toro $T$ obtido pela identificação dos segmentos de mesmo nome, como mostra a Figura 4.5, respeitando a orientação dos mesmos. Então,

$$
\chi(T)=9-27+18=0 .
$$

Exemplo 4.3.10. Consideremos $M$ o toro pinçado, obtido pela identificação dos vértices e dos segmentos de mesmo nome, como mostra a Figura 4.6, respeitando a orientação dos mesmos. Assim,

$$
\chi(M)=7-18+12=1 .
$$




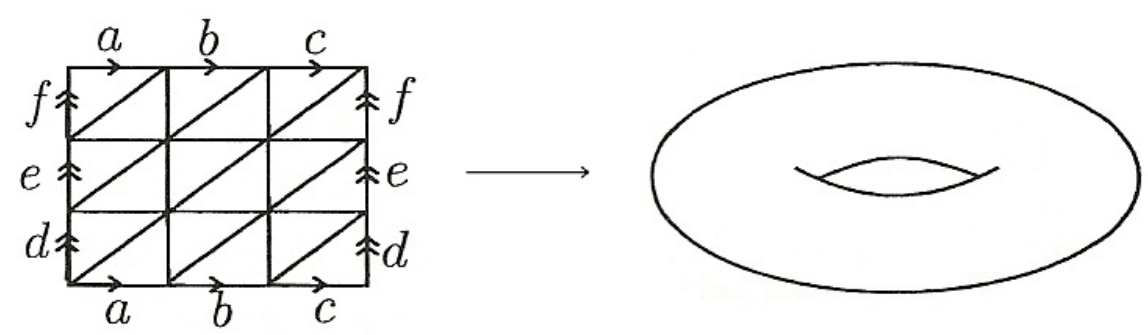

Figura 4.5: Triangularização do toro $T$

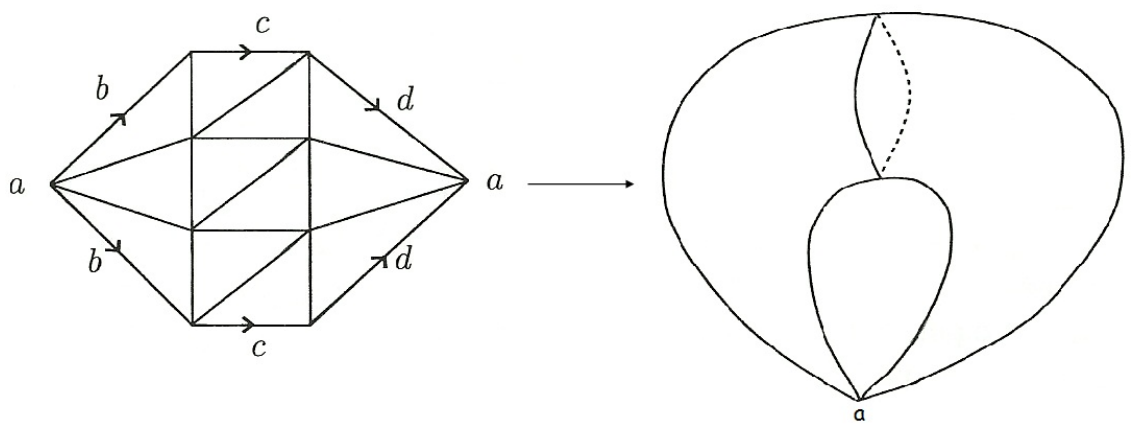

Figura 4.6: Triangularização do toro pinçado $M$

Observação 4.3.11. Dada $M$ uma variedade diferenciável compacta e orientada, a característica de Euler de $M, \chi(M)$, mede a "obstrução" para construírmos um campo de vetores continuo sobre $M$ sem singularidades. Mais precisamente, temos que existe um campo de vetores contínuo $v$ sobre $M$, sem singularidades, se, e somente se, $\chi(M)=$ 0. Este resultado, é na verdade uma consequência do Teorema de Poincaré-Hopf, que demonstraremos na próxima seção.

\subsection{O Teorema de Poincaré-Hopf}

Nesta seção, demonstraremos o Teorema de Poincaré-Hopf, que associa a soma dos índices das singularidades isoladas de um campo de vetores contínuo sobre uma variedade diferenciável compacta $M$ com a característica de Euler de $M$, este teorema é o mais importante deste capítulo. Para sua demonstração necessitaremos de vários teoremas provados anteriormente e de uma série de resultados que apresentaremos a seguir. 
Lema 4.4.1. Sejam $\mathbb{S}_{1}, \mathbb{S}_{2} \subset \mathbb{R}^{m}$ esferas, $(m-1)$-dimensionais, mutuamente exteriores, de centros $a_{1}, a_{2}$ respectivamente, contidas no interior de uma esfera $\mathbb{S}_{0}$. Sejam $f: \mathbb{R}^{m} \backslash\left\{a_{1}, a_{2}\right\} \rightarrow \mathbb{S}^{m-1}$ uma aplicação contínua e $f_{i}=\left.f\right|_{\mathbb{S}_{i}}, i=0,1,2$. Se orientarmos igualmente as esferas $\mathbb{S}_{i}$, teremos $g r\left(f_{0}\right)=\operatorname{gr}\left(f_{1}\right)+g r\left(f_{2}\right)$.

Demonstração: Primeiramente mostraremos o caso em que a aplicação $f$ é diferenciável.

Seja $\overline{\mathbb{B}}$ uma bola fechada contida em $\mathbb{R}^{m} \backslash\left\{a_{1}, a_{2}\right\}$, tal que $f(\overline{\mathbb{B}})$ não cubra toda a esfera $\mathbb{S}^{m-1}$.

Mediante uma deformação, podemos transformar as esferas dadas em elipsóides, $\mathbb{S}_{0}^{1}$, $\mathbb{S}_{1}^{1}, \mathbb{S}_{2}^{1}$, como ilustra a Figura 4.7 , de modo que as esferas não toquem $a_{1}$ nem $a_{2}$ durante

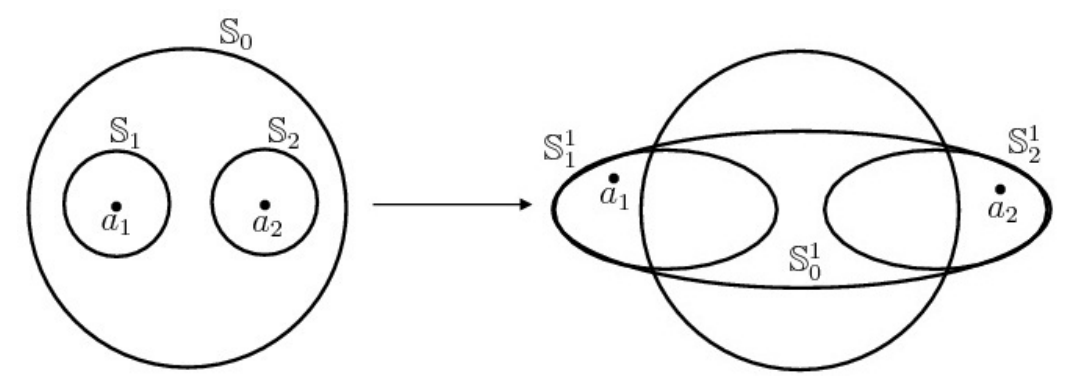

Figura 4.7: Deformação das esferas $\mathbb{S}_{0}, \mathbb{S}_{0}$ e $\mathbb{S}_{0}$

a deformação e, no final, $\mathbb{S}_{0}^{1}$ coincida com $\mathbb{S}_{1}^{1} \cup \mathbb{S}_{2}^{1}$ fora de $\overline{\mathbb{B}}$. Mais precisamente, devemos ter

$$
\mathbb{S}_{0}^{1} \cap \overline{\mathbb{B}}^{c}=\left(\mathbb{S}_{1}^{1} \cap \overline{\mathbb{B}}^{c}\right) \cup\left(\mathbb{S}_{2}^{1} \cap \overline{\mathbb{B}}^{c}\right)
$$

Definindo $f_{i}^{1}=\left.f\right|_{\mathbb{S}_{i}^{1}}, i=0,1,2$, temos $\operatorname{gr}\left(f_{i}\right)=\operatorname{gr}\left(f_{i}^{1}\right)$, pois o grau não se altera por deformações.

Seja agora $c \in \mathbb{S}^{m-1}$ um valor regular de $f_{0}^{1}$, com $c \in \mathbb{S}^{m-1} \backslash f(\overline{\mathbb{B}})$. Como $\mathbb{S}^{m-1} \backslash f(\overline{\mathbb{B}})$ é não vazio, tal ponto $c$ existe devido ao teorema de Sard. Além disso, como $f^{-1}(c) \subset \overline{\mathbb{B}}^{c}$ e, fora de $\overline{\mathbb{B}}, f_{0}^{1}$ coincide com $f_{1}^{1}$ ou $f_{2}^{1}$, vemos que $c$ é valor regular de $f_{1}^{1}$ e de $f_{2}^{1}$, e que

$$
\left(f_{0}^{1}\right)^{-1}(c)=\left(f_{1}^{1}\right)^{-1}(c) \dot{\cup}\left(f_{2}^{1}\right)^{-1}(c) .
$$

Logo,

$$
\operatorname{gr}\left(f_{0}^{1}\right)=\operatorname{gr}\left(f_{1}^{1}\right)+\operatorname{gr}\left(f_{2}^{1}\right)
$$


como queríamos mostrar.

Finalmente, consideremos o caso em que a aplicação $f: \mathbb{R}^{m} \backslash\left\{a_{1}, a_{2}\right\} \rightarrow \mathbb{S}^{m-1}$ é apenas contínua. Tomamos uma aplicação diferenciável $g: \mathbb{R}^{m} \backslash\left\{a_{1}, a_{2}\right\} \rightarrow \mathbb{S}^{m-1}$ tal que $\|f(x)-g(x)\|<2$ para $x \in \mathbb{S}_{0} \cup \mathbb{S}_{1} \cup \mathbb{S}_{2}$, isto é, tal que

$$
\left.\left.f\right|_{\mathbb{S}_{0} \cup \mathbb{S}_{1} \cup \mathbb{S}_{2}} \simeq g\right|_{\mathbb{S}_{0} \cup \mathbb{S}_{1} \cup \mathbb{S}_{2}}
$$

o que sempre podemos conseguir devido ao Teorema 1.2.6.

Definindo $g_{i}=\left.g\right|_{\mathbb{S}_{i}}$, temos que $g_{i} \simeq f_{i}: \mathbb{S}_{i} \rightarrow \mathbb{S}^{m-1}$, de onde $\operatorname{gr}\left(g_{i}\right)=g r\left(f_{i}\right)$, recaindo assim no caso anterior.

Observação 4.4.2. Utilizando um processo análogo ao da demonstração do Lema 4.4.1. podemos generalizar este resultado para $r>2$, isto é, dadas $\mathbb{S}_{1}, \ldots, \mathbb{S}_{r} \subset \mathbb{R}^{m}$ esferas, $(m-1)$-dimensionais, mutuamente exteriores, de centro $a_{1}, \ldots, a_{r}$ respectivamente, contidas no interior de uma esfera $\mathbb{S}_{0}$. Sejam $f: \mathbb{R}^{m} \backslash\left\{a_{1}, \ldots, a_{r}\right\} \rightarrow \mathbb{S}^{m-1}$ uma aplicação continua e $f_{i}=\left.f\right|_{\mathbb{S}_{i}}, i=0, \ldots, r$. Se orientarmos igualmente as esferas $\mathbb{S}_{i}$, teremos $\operatorname{gr}\left(f_{0}\right)=\operatorname{gr}\left(f_{1}\right)+\ldots+\operatorname{gr}\left(f_{r}\right)$.

Teorema 4.4.3. Sejam $v$ um campo vetorial contínuo sobre uma variedade $m$-dimensional $M, p_{1}$ e $p_{2}$ pertencentes a $M$ duas singularidades isoladas de $v$. Dado um aberto conexo $V \subset M$ tal que $p_{1}, p_{2} \in V$ são as únicas singularidades de $v$ em $V$, existe um campo vetorial contínuo $u$ sobre $M$, que coincide com $v$ em $M \backslash V$, e que admite apenas uma singularidade $p$ em $V$. Além disso, temos

$$
\operatorname{Ind}_{P H}(u, p)=\operatorname{Ind}_{P H}\left(v, p_{1}\right)+\operatorname{Ind}_{P H}\left(v, p_{2}\right) .
$$

Demonstração: Como $V$ é conexo, pelo Teorema 1.1.12, existe uma carta $\varphi: U \rightarrow \mathbb{R}^{m}$, onde $U$ é um aberto de $M$ contido em $V$ tal que $p_{1}, p_{2} \in U$ e $\varphi(U)=\mathbb{R}^{m}$. Sejam $a_{1}=\varphi\left(p_{1}\right)$ e $a_{2}=\varphi\left(p_{2}\right)$ e consideremos uma bola fechada, centrada em $0 \in \mathbb{R}^{m}$ e de raio $r$, que denotaremos por $\overline{\mathbb{B}}_{r}(0) \subset \mathbb{R}^{m}$, contendo os pontos $a_{1}$ e $a_{2}$ em seu interior. A expressão

$$
v_{q}=\sum_{i=1}^{m} \alpha_{i}(\varphi(q)) \frac{\partial}{\partial x_{i}}(q), \quad q \in U
$$


do campo $v$ em termos da carta $\varphi$, nos fornece uma aplicação contínua $f: \mathbb{R}^{m} \rightarrow \mathbb{R}^{m}$, dada por

$$
f(x)=\left(\alpha_{1}(x), \ldots, \alpha_{m}(x)\right), \quad x \in \mathbb{R}^{m}
$$

a qual assume o valor $f(x)=0$ apenas para $x=a_{1}$ e $x=a_{2}$. Em particular, $f(x) \neq 0$ sempre que $\|x\|=r$, isto é, sempre que $x$ pertence ao bordo de $\overline{\mathbb{B}}_{r}(0)$. Definimos uma nova aplicação $g: \mathbb{R}^{m} \rightarrow \mathbb{R}^{m}$, pondo

$$
g(x)=\left\{\begin{array}{l}
f(x), \text { se }\|x\| \geq r \\
\frac{\|x\|}{r} f\left(\frac{r}{\|x\|} x\right), \text { se } 0<\|x\| \leq r \\
0, \text { se } x=0
\end{array}\right.
$$

Então $g: \mathbb{R}^{m} \rightarrow \mathbb{R}^{m}$ é contínua, coincide com $f$ fora da bola $\overline{\mathbb{B}}_{r}(0)$, e assume o valor $0 \in \mathbb{R}^{m}$ somente no ponto $0 \in \mathbb{R}^{m}$. Sejam $\beta_{1}, \ldots, \beta_{m}: \mathbb{R}^{m} \rightarrow \mathbb{R}$ as funções contínuas tais que

$$
g(x)=\left(\beta_{1}(x), \ldots, \beta_{m}(x)\right)
$$

e definamos um campo vetorial $u$ sobre $M$, pondo $u_{q}=v_{q}$ se $q \in M \backslash U$, e

$$
u_{q}=\sum_{i=1}^{m} \beta_{i}(\varphi(q)) \frac{\partial}{\partial x_{i}}(q) \text {, }
$$

se $q \in U$. Temos que $u$ é contínuo, coincide com $v$ em $M \backslash V$ e a única singularidade de $u$ em $V$ é o ponto $p=\varphi^{-1}(0)$.

Agora, resta mostrarmos que $\operatorname{Ind}_{P H}(u, p)=\operatorname{Ind}_{P H}\left(v, p_{1}\right)+\operatorname{Ind}_{P H}\left(v, p_{2}\right)$.

Para isso, consideremos $\overline{\mathbb{B}}_{\varepsilon_{1}}\left(a_{1}\right)$ e $\overline{\mathbb{B}}_{\varepsilon_{2}}\left(a_{2}\right)$ bolas fechadas disjuntas, de centro $a_{1}$, $a_{2}$ e raio $\varepsilon_{1}, \varepsilon_{2}$, respectivamente, contidas na bola $\overline{\mathbb{B}}_{r}(0)$. Sejam $\mathbb{S}_{0}, \mathbb{S}_{1}$ e $\mathbb{S}_{2}$ as esferas que servem de bordo às bolas $\overline{\mathbb{B}}_{r}(0), \overline{\mathbb{B}}_{\varepsilon_{1}}\left(a_{1}\right)$ e $\overline{\mathbb{B}}_{\varepsilon_{2}}\left(a_{2}\right)$, respectivamente. Indiquemos por

$$
j_{0}: \mathbb{S}^{m-1} \rightarrow \overline{\mathbb{B}}_{r}(0) \backslash\{0\}, \quad j_{1}: \mathbb{S}^{m-1} \rightarrow \overline{\mathbb{B}}_{\varepsilon_{1}}\left(a_{1}\right) \backslash\left\{a_{1}\right\}, \quad j_{2}: \mathbb{S}^{m-1} \rightarrow \overline{\mathbb{B}}_{\varepsilon_{2}}\left(a_{2}\right) \backslash\left\{a_{2}\right\}
$$


as equivalências homotópicas descritas na Seção 3.2. Temos

$$
\begin{gathered}
\operatorname{Ind}_{P H}\left(v, p_{1}\right)=g r\left(\rho \circ f \circ j_{1}\right), \\
\operatorname{Ind}_{P H}\left(v, p_{2}\right)=\operatorname{gr}\left(\rho \circ f \circ j_{2}\right), \\
\operatorname{Ind}_{P H}(u, p)=\operatorname{gr}\left(\rho \circ g \circ j_{0}\right)=g r\left(\rho \circ f \circ j_{0}\right),
\end{gathered}
$$

observamos que a última igualdade é válida, pois $\left.g\right|_{\mathbb{S}_{0}}=\left.f\right|_{\mathbb{S}_{0}}$, uma vez que $j_{0}\left(\mathbb{S}^{m-1}\right)=\mathbb{S}_{0}$. O resultado segue então do Lema 4.4.1.

Corolário 4.4.4. Seja $v$ um campo vetorial contínuo sobre uma variedade diferenciável $m$-dimensional $M$, admitindo um número finito de singularidades $p_{1}, \ldots, p_{r}$. Dado um aberto conexo $V$, contendo todas estas singularidades, existe um campo vetorial contínuo $u$ sobre $M$, que coincide com $v$ em $M \backslash V$, e que possui apenas uma singularidade $p \in M$, com

$$
\operatorname{Ind}_{P H}(u, p)=\sum_{i=1}^{r} \operatorname{Ind}_{P H}\left(v, p_{i}\right) .
$$

Demonstração: Consideremos primeiramente as singularidades $p_{1}$ e $p_{2}$, seja $V_{12} \subset V$ um aberto conexo dentro do qual as únicas singularidades de $v$ são $p_{1}$ e $p_{2}$.

Aplicando o Teorema 4.4.3, obtemos um campo contínuo $u_{1}$ sobre $M$, coincidindo com $v$ em $M \backslash V_{12}$ e, consequentemente em $M \backslash V$, o qual possui somente uma singularidade $q_{2} \in V_{12}, \mathrm{com}$

$$
\operatorname{Ind}_{P H}\left(u_{1}, q_{2}\right)=\operatorname{Ind}_{P H}\left(v, p_{1}\right)+\operatorname{Ind}_{P H}\left(v, p_{2}\right) .
$$

Em seguida, consideremos um aberto conexo $V_{23} \subset V$ contendo $q_{2}, p_{3}$, e nenhuma outra singularidade de $u_{1}$. Aplicando novamente o Teorema 4.4.3, obtemos um campo vetorial contínuo $u_{2}$ sobre $M$, coincidinco com $u_{1}$ em $M \backslash V_{23}$ e, consequentemente com $v$ em $M \backslash V$, o qual possui apenas uma singularidade $q_{3} \in V_{23}$, com

$$
\begin{aligned}
\operatorname{Ind}_{P H}\left(u_{2}, q_{3}\right) & =\operatorname{Ind}_{P H}\left(u_{1}, q_{2}\right)+\operatorname{Ind}_{P H}\left(u_{1}, p_{3}\right) \\
& =\operatorname{Ind} d_{P H}\left(v, p_{1}\right)+\operatorname{Ind}_{P H}\left(v, p_{2}\right)+\operatorname{Ind}_{P H}\left(v, p_{3}\right) .
\end{aligned}
$$

Prosseguindo analogamente, obtemos no final um campo contínuo $u=u_{r-1}$ sobre $M$, o qual coincide com $v$ em $M \backslash V$, e admite apenas uma singularidade $p=q_{r}$ em $M$, sendo 
seu índice

$$
\operatorname{Ind}_{P H}(u, p)=\sum_{i=1}^{r} \operatorname{Ind}_{P H}\left(v, p_{i}\right)
$$

como queríamos demonstrar.

Uma das propriedades do índice de Poincaré-Hopf é estabilidade sobre perturbações. Em outras palavras, se um campo de vetores $v$ definido em uma variedade diferenciável $M$ tem uma singularidade isolada e se o perturbamos levemente, então a singularidade $p$ do campo de vetores inicial $v$ poderá se dividir em vários pontos singulares, com a propriedade que a soma dos índices do campo de vetores perturbado em seus pontos singulares é igual ao índice do campo de vetores original $v$ em sua singularidade.

Teorema 4.4.5. Sejam uma variedade diferenciável compacta e orientada $M$, e $v: M \rightarrow$ $T M$ um campo de vetores diferenciável, com uma quantidade finita de singularidades isoladas $p_{1}, \ldots, p_{r}$. Então, existe um campo vetorial diferenciável $w$ cujas singularidades são todas simples e tal que a soma dos índices das singularidades de $v$ é igual a soma dos índices das singularidades simples de $w$.

Demonstração: Pelo Corolário 4.4.4, existe um campo vetorial contínuo $u$ sobre $M$, que possui somente uma singularidade $p$, satisfazendo

$$
\operatorname{Ind}_{P H}(u, p)=\sum_{i=1}^{r} \operatorname{Ind}_{P H}\left(v, p_{i}\right) .
$$

Pelo Teorema 1.1.12, existe $\varphi: V \rightarrow \mathbb{R}^{m}$ uma carta de $M$, onde $\varphi(V)=\mathbb{R}^{m}$ com $p \in V$. Tomemos uma bola fechada $\overline{\mathbb{B}}(a) \subset \mathbb{R}^{m}$, de centro $a=\varphi(p)$ e chamemos de $\mathbb{S}$ a esfera que serve de bordo a $\overline{\mathbb{B}}(a)$. Aplicando o Teorema 4.1.16, tomemos um campo vetorial diferenciável $w$ sobre $M$, que possua somente singularidades simples, e está tão próximo de $u$ que satisfaça às condições seguintes:

1) não existem singularidades de $w$ em $M \backslash \varphi^{-1}(\mathbb{B}(a))$; 
2) se $f, g: \mathbb{R}^{m} \rightarrow \mathbb{R}^{m}$ são as aplicações definidas por $f(x)=\left(\alpha_{1}(x), \ldots, \alpha_{m}(x)\right)$ e $g(x)=\left(\beta_{1}(x), \ldots, \beta_{m}(x)\right)$, onde

$$
u=\sum_{j=1}^{m} \alpha_{j} \frac{\partial}{\partial x_{j}} \text { e } w=\sum_{j=1}^{m} \beta_{j} \frac{\partial}{\partial x_{i}} \text { em } V
$$

então, $\left|\frac{f(x)}{|f(x)|}-\frac{g(x)}{|g(x)|}\right|<2$, para todo $x \in \mathbb{S}$.

Da última condição, temos que

$$
\rho \circ f \circ j \simeq \rho \circ g \circ j: \mathbb{S}^{m-1} \rightarrow \mathbb{S}^{m-1},
$$

(ver Exemplo 1.2.3) consequentemente, pelo Teorema 3.1.19, temos

$$
\operatorname{Ind}_{P H}(u, p)=\gamma_{a}(f)=\operatorname{gr}(\rho \circ f \circ j)=\operatorname{gr}(\rho \circ g \circ j) .
$$

Assim, $\operatorname{Ind}_{P H}(u, p)$ é igual ao grau da restrição $\left.(\rho \circ g)\right|_{\mathbb{S}}$, onde $g$ é definida pelo campo $w$ em termos da carta $\varphi$.

O campo $w$ possui apenas um número finito de singularidades $q_{1}, \ldots, q_{s}$ em $M$, as quais pertencem a $\varphi(\mathbb{B}(a))$, sejam $a_{1}=\varphi\left(q_{1}\right), \ldots, a_{s}=\varphi\left(q_{s}\right) \in \mathbb{B}(a)$ e tomemos pequenas esferas disjuntas $\mathbb{S}_{j}$, de centro $a_{j}$, contidas em $\mathbb{S}$. Considerando a aplicação

$$
h=\rho \circ g: \mathbb{R}^{m} \backslash\left\{a_{1}, \ldots, a_{s}\right\} \rightarrow \mathbb{S}^{m-1},
$$

temos

$$
\operatorname{Ind}_{P H}(u, p)=\operatorname{gr}\left(\left.h\right|_{\mathbb{S}}\right) \text { e } \operatorname{Ind}_{P H}\left(w, q_{j}\right)=\operatorname{gr}\left(\left.h\right|_{\mathbb{S}_{j}}\right) .
$$

Mas, pelo Lema 4.4.1), (ver Observação 4.4.2) vale

$$
\operatorname{gr}\left(\left.h\right|_{\mathbb{S}}\right)=\sum_{j=1}^{s} g r\left(\left.h\right|_{\mathbb{S}_{j}}\right),
$$


o que implica que $\operatorname{Ind}_{P H}(u, p)=\sum_{j=1}^{s} g r\left(\left.h\right|_{\mathbb{S}_{j}}\right)$, portanto

$$
\sum_{i=1}^{r} \operatorname{Ind}_{P H}\left(v, p_{i}\right)=\sum_{j=1}^{s} \operatorname{Ind}_{P H}\left(w, q_{j}\right)
$$

Como queríamos demonstrar.

Observação 4.4.6. O teorema acima, associa a soma dos indices das singularidades $p_{1}, \ldots, p_{r}$ do campo $v$ com a soma dos indices das singularidades simples $q_{1}, \ldots, q_{s}$ do campo w. Mas, lembremos que o índice de uma singularidade simples é sempre 1 ou -1. Então, do teorema acima, podemos concluir que o indice de cada singularidade $p_{i}$ do campo $v$, será igual a soma dos indices de uma certa quantidade de singularidades de $w$.

Na Seção 4.3, definimos a característica de Euler de uma variedade diferenciável, a seguir, mostraremos que dada uma variedade diferenciável compacta $M$ sua característica de Euler $\chi(M)$ é igual a soma dos índices de Poincaré-Hopf de um campo de vetores diferenciável cujas singularidades são todas simples, independente da escolha do campo nestas condições.

Teorema 4.4.7. Sejam uma variedade diferenciável compacta e orientada $M$, e $v: M \rightarrow$ $T M$ um campo de vetores diferenciável cujas singularidades são todas simples. Então, a característica de Euler de $M$ é igual a soma dos índices de Poincaré-Hopf de todas as singularidade de $v$, isto é, $\chi(M)=\sum_{i=1}^{r} \operatorname{Ind}_{P H}\left(v, p_{i}\right)$, onde $p_{i} \in M$ são as singularidades de $v$.

Não faremos aqui uma demonstração completa deste resultado, pois para isso são necessários recursos da teoria de cohomologia. Apresentaremos aqui a idéia geométrica da demonstração, no caso em que $M$ é uma superfície, ou seja, uma variedade de dimensão 2 .

Demonstração: Seja $M$ uma variedade diferenciável 2-dimensional, compacta e orientada, queremos mostrar que dado um campo de vetores diferenciável $v: M \rightarrow T M$, cujas singularidades são todas simples, a característica de Euler de $M$ é igual a soma dos índices de Poincaré-Hopf de todas as singularidade de $v$. 
Se mostrarmos que existe um campo de vetores $v$, nas condições anteriores, que satisfaça essa igualdade o resultado seguirá do Teorema 4.2.10. Mostremos então que tal campo existe.

De fato, dada $M$ uma variedade compacta e orientável de dimensão 2 consideremos uma triangulação de $M$, denotemos por $n_{i}$, o número de simplexos $i$-dimensionais desta triangulação, onde $i=0,1,2$.

Subdividindo baricentricamente cada triângulo de $M$, isto é, subdividindo cada triângulo de $M$ em 6 outros triângulos traçando suas 3 medianas. Em seguida, enchemos o triângulo com linhas integrais do campo, cada linha integral partindo sempre do centro de um elemento de dimensão menor para o centro de um elemento de dimensão maior, ou seja, de um vértice para o meio de um lado, de um vértice para o centro de um triângulo, ou do meio de um lado para o centro de um triângulo.

Cada elemento, vértice, aresta ou face, contribui precisamente com uma singularidade do campo, o centro deste elemento é um ponto singular. Assim, tal campo terá $n_{0}+n_{1}+n_{2}$ singularidades.

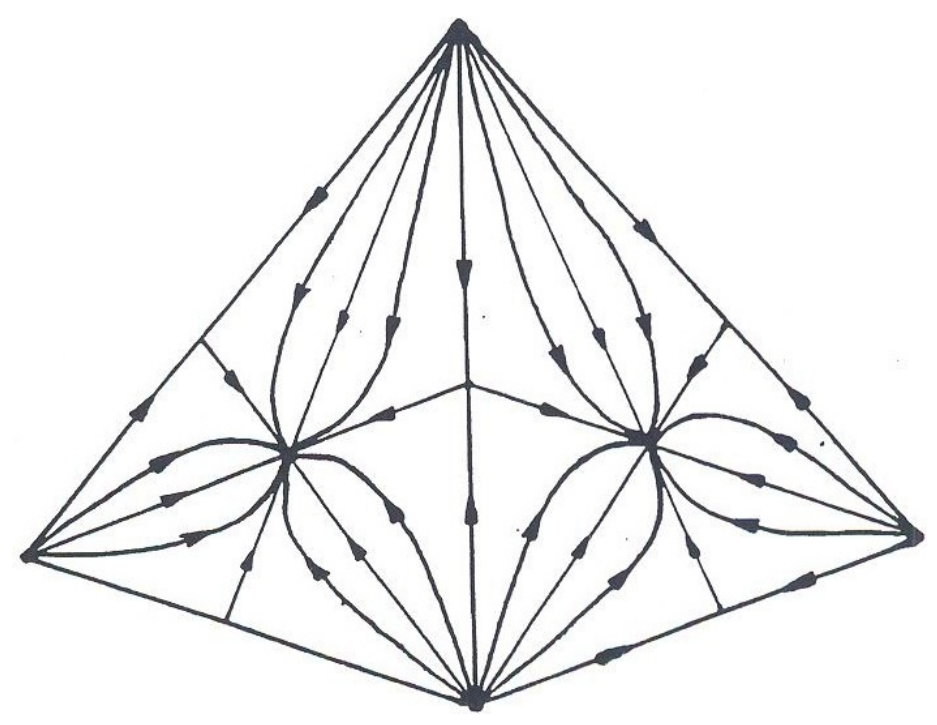

Figura 4.8: Campo de Hopf

Observemos, que a situação geométrica apresentada neste campo é a mesma apresentada no Exemplo 2.1.6. Assim, as singularidades deste campo tem sempre índice +1 ou -1 , além disso, podemos supor que as singularidades deste campo são simples. 
Agora, notemos que a soma dos índices das singularidades deste campo é $n_{0}-n_{1}+n_{2}$, que é exatamente a característica de Euler de $M$.

Corolário 4.4.8. Seja $M$ uma variedade diferenciável $m$-dimensional, compacta e orientada. Se $m$ é ímpar, então $\chi(M)=0$.

Demonstração: Seja $v: M \rightarrow T M$ um campo vetorial diferenciável, cujas singularidades $p_{1}, \ldots, p_{r}$ são todas simples. As singularidades do campo vetorial diferenciável $-v$ são as mesmas que as do campo $v$ e também são todas simples. Então, pelo Teorema 4.2.11 temos

$$
\sum_{i=1}^{r} \operatorname{Ind}_{P H}\left(v, p_{i}\right)=\sum_{i=1}^{r} \operatorname{Ind}_{P H}\left(-v, p_{i}\right) .
$$

Se consideremos uma carta $\varphi_{i}: V_{i} \rightarrow U_{i}$ de $M$, onde $V_{i}$ é um aberto de $M$ e $U_{i}$ é um aberto de $\mathbb{R}^{m}$, tal que, a singularidade $p_{i}$ do campo $v$ esteja contida em $V_{i}$, então podemos escrever

$$
v_{q}=\sum_{i=1}^{m} \alpha_{i}(q) \frac{\partial}{\partial x_{i}}(q) .
$$

para todo $q \in V_{i}$. Assim, o índice de Poincaré-Hopf da singularidade $p_{i}$ em relação ao campo $v$ é o sinal do determinante

$$
\operatorname{det}\left(\frac{\partial \alpha_{i}}{\partial x_{j}}\left(p_{i}\right)\right)
$$

enquanto o índice de $p_{i}$ em relação a $-v$ é o sinal de

$$
\operatorname{det}\left(-\frac{\partial \alpha_{i}}{\partial x_{j}}\left(p_{i}\right)\right)=(-1)^{m} \operatorname{det}\left(\frac{\partial \alpha_{i}}{\partial x_{j}}\left(p_{i}\right)\right)
$$

Então, podemos dizer que

$$
\sum_{i=1}^{r} \operatorname{Ind}_{P H}\left(v, p_{i}\right)=(-1)^{m} \sum_{i=1}^{r} \operatorname{Ind}_{P H}\left(-v, p_{i}\right),
$$

como $m$ é ímpar e por 4.4.1), segue que $\sum_{i=1}^{r} \operatorname{Ind}_{P H}\left(v, p_{i}\right)=0$. Portanto, do Teorema 4.4.7), temos $\chi(M)=0$. 
Agora, que fizemos as considerações necessárias, provaremos o Teorema de PoincaréHopf, que é um dos resultados principais deste trabalho.

Os esforços feitos para provarmos os resultados anteriores foram necessários para que pudessemos apresentar uma demonstração mais clara e objetiva deste teorema.

Teorema 4.4.9. (Poincaré-Hopf) Seja $M$ uma variedade diferenciável compacta e orientada, dado $v$ um campo vetorial contínuo sobre $M$, com um número finito de singularidades $p_{1}, \ldots, p_{r}$. Então,

$$
\chi(M)=\sum_{i=1}^{r} \operatorname{Ind}_{P H}\left(v, p_{i}\right)
$$

Demonstração: No Teorema 4.4.5, mostramos que existe um campo de vetores diferenciável $w: M \rightarrow T M$, cujas as singularidades $q_{1}, \ldots, q_{s}$ são todas simples e tal que

$$
\sum_{i=1}^{r} \operatorname{Ind}_{P H}\left(v, p_{i}\right)=\sum_{j=1}^{s} \operatorname{Ind}_{P H}\left(w, q_{j}\right) .
$$

Portanto, do Teorema 4.4.7, segue que

$$
\sum_{i=1}^{r} \operatorname{Ind}_{P H}\left(v, p_{i}\right)=\chi(M)
$$

Como queríamos demonstrar.

O Teorema de Poincaré-Hopf, foi demonstrado em dimensão 2 por H. Poincaré em 1885, e generalizado para dimensões maiores por H. Hopf em 1927. E significa que a característica de Euler calcula a obstrução para a construção de um campo de vetores tangente a $M$, sem singularidades. Como mostra o teorema abaixo.

Teorema 4.4.10. Seja $M$ uma variedade diferenciável compacta, existe um campo vetorial contínuo sem singularidades sobre $M$ se, e somente se, $\chi(M)=0$.

Demonstração: Suponhamos que exista um campo vetorial contínuo sem singularidades, pelo Teorema 4.4.7, temos que $\chi(M)=0$. 
Suponhamos agora, que $\chi(M)=0$, então existe um campo vetorial diferenciável $v$ sobre $M$, cujas singularidades $p_{1}, \ldots, p_{r}$ são todas simples, e

$$
\sum_{i=1}^{r} \operatorname{Ind}_{P H}\left(v, p_{i}\right)=\chi(M)=0
$$

Usando o Corolário 4.4.4 obtemos um campo contínuo $u$ sobre $M$, com uma única singularidade $p \in M$, tal que $\operatorname{Ind}_{P H}(u, p)=0$. Seja $\varphi: V \rightarrow \mathbb{R}^{m}$ uma carta de $M$, com $p \in V$ e $\varphi(V)=\mathbb{R}^{m}$, assim podemos escrever

$$
u_{q}=\sum_{i=1}^{m} \alpha_{i}(\varphi(q)) \frac{\partial}{\partial x_{i}}(q), \quad q \in V
$$

Isto define uma aplicação $f: \mathbb{R}^{m} \rightarrow \mathbb{R}^{m}$, onde

$$
f(x)=\left(\alpha_{1}(x), \ldots, \alpha_{m}(x)\right)
$$

Para $a=\varphi(p)$, temos $f(a)=0 \in \mathbb{R}^{m}$, onde $f\left(\mathbb{R}^{m} \backslash\{a\}\right) \subset \mathbb{R}^{m} \backslash\{0\}$, e $\gamma_{a}(f)=0$.

Tomemos uma bola fechada $\overline{\mathbb{B}}_{\varepsilon}(a)$ de centro $a$ em $\mathbb{R}^{m}$ e raio $\varepsilon$. Pelo Teorema 3.2.10. existe uma aplicação contínua $g: \mathbb{R}^{m} \rightarrow \mathbb{R}^{m}$, que coincide com $f$ em $\mathbb{R}^{m} \backslash \mathbb{B}_{\varepsilon}(a)$, seja

$$
g(x)=\left(\beta_{1}(x), \ldots, \beta_{m}(x)\right), \quad x \in \mathbb{R}^{m} .
$$

Definimos um campo vetorial $w$ sobre $M$, pondo

$$
w_{q}=v_{q} \text { se } q \in M \backslash\left\{\varphi^{-1}\left(\overline{\mathbb{B}}_{\varepsilon}(a)\right)\right\}
$$

e

$$
w_{q}=\sum_{i=1}^{m} \beta_{i}(\varphi(q)) \frac{\partial}{\partial x_{i}}(q) \text { se } q \in \varphi^{-1}\left(\overline{\mathbb{B}}_{\varepsilon}(a)\right) .
$$

Portanto, o campo $w$ é contínuo e não possui singularidades.

Exemplo 4.4.11. Nos Exemplos 4.3.8 e 4.3.9 vimos que a característica de Euler da esfera $\mathbb{S}^{2}$ e do toro $T$ contido em $\mathbb{R}^{3}$ são $\chi\left(\mathbb{S}^{2}\right)=2$ e $\chi(T)=0$, respectivamente. Então, do Teorema de Poincaré-Hopf, segue que existe um campo de vetores tangente ao toro $T$ 
que não possui singularidades, porém todo campo de vetores sobre a esfera $\mathbb{S}^{2}$ apresentará pelo menos uma singularidade.

A seguir, apresentamos representações geométricas de dois campos de vetores, um sobre a esfera, contendo singularidades, e outro sobre o toro, sem singularidades.

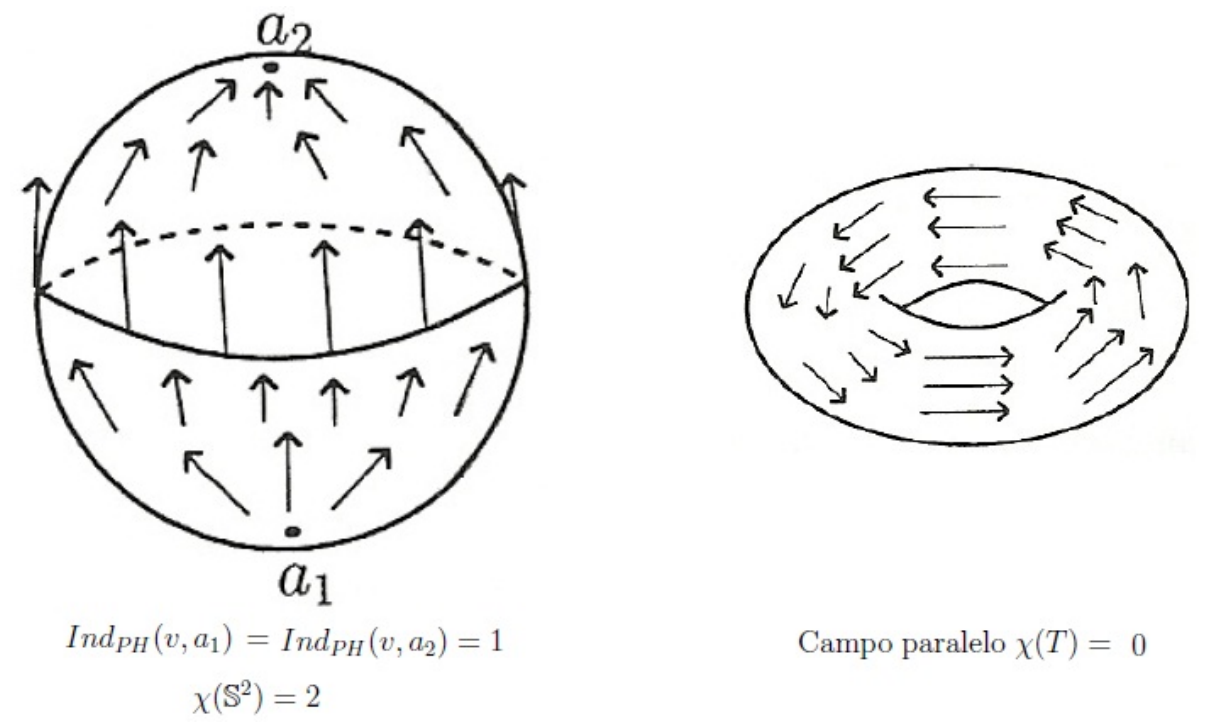

Figura 4.9: Teorema de Poincaré-Hopf para a esfera e o toro.

Se $M$ é uma variedade diferenciável com bordo, temos o similar teorema.

Teorema 4.4.12. Sejam $M$ uma variedade diferenciável, compacta, orientada e com bordo $\partial M$, e $v$ um campo de vetores sem singularidades em uma vizinhança $U$ de $\partial M$. Então:

i) O campo de vetores $v$ pode ser estendido para o interior de $M$ com uma quantidade finita de singularidades;

ii) O índice de Poincaré-Hopf total de $v$ em $M$ é independente da maneira como estendemos o campo $v$ para o interior de $M$;

iii) Seja $\tilde{v}$ uma extensão de $v$ em $M$, se $\tilde{v}$ é transversal ao bordo de $M$ e aponta para "fora" de $M$, então temos que $\operatorname{Ind}_{P H}(\tilde{v}, M)=\chi(M)$. Se $\tilde{v}$ é transversal a $\partial M$ e aponta para "dentro" de $M$, então $\operatorname{Ind}_{P H}(\tilde{v}, M)=\chi(M)-\chi(\partial M)$. 
O teorema acima pode ser visto em [3].

Sejam $M$ uma variedade diferenciável, $v$ um campo de vetores sobre $M$ com uma singularidade isolada em $p$ e $\overline{\mathbb{B}}_{\varepsilon}(p)$ uma bola em $M$ (para alguma métrica) de centro $p$ e raio $\varepsilon$. A classe dos campos de vetores, com singularidade isolada em $p$, que são transversais ao bordo de $\overline{\mathbb{B}}_{\varepsilon}(p)$, desempenharão um papel fundamental na Seção 5.1. Então, calculemos o índice da singularidade $p$ de tais campos.

Proposição 4.4.13. Sejam $M$ uma variedade diferenciável $m$-dimensional orientada e $v: M \rightarrow T M$ um campo de vetores com uma singularidade isolada em $p$, tal que, $v$ seja transversal ao bordo da bola $\overline{\mathbb{B}}_{\varepsilon}(p)$ de centro $p$ e raio $\varepsilon$ em $M$. Então:

i) Se $v$ aponta para "fora" de $\mathbb{S}_{\varepsilon}$, então $\operatorname{Ind}_{P H}(v, p)=+1$;

ii) Se $v$ aponta para "dentro" de $\mathbb{S}_{\varepsilon}$, então $\operatorname{Ind}_{P H}(v, p)=+1$ se $m$ é par, no caso em que $m$ é ímpar temos $\operatorname{Ind}_{P H}(p, v)=-1$.

Basta provarmos o caso em que $M=\mathbb{R}^{m}$, o caso geral, segue deste e da definição de índice.

Demonstração: Seja $\overline{\mathbb{B}}_{\varepsilon}(p)$ a bola fechada de centro $p$ e raio $\varepsilon$ de $\mathbb{R}^{m}$ (com a métrica usual). Da Observação 3.2 .4 temos que $\operatorname{Ind}_{P H}(v, p)=\operatorname{Ind}_{P H}(w, p)$, onde $w=\left.v\right|_{\overline{\mathbb{B}}_{\varepsilon}(p)}$.

i) Se $w$ é transversal a $\mathbb{S}_{\varepsilon}$ e aponta para "fora", pelo Teorema 4.4.12, segue que

$$
\chi\left(\overline{\mathbb{B}}_{\varepsilon}(p)\right)=\operatorname{Ind}_{P H}(w, p),
$$

$\operatorname{mas} \chi\left(\overline{\mathbb{B}}_{\varepsilon}(p)\right)=1$, portanto, $\operatorname{Ind}_{P H}(w, p)=\operatorname{Ind}_{P H}(v, p)=1$;

ii) Se $w$ é transversal a $\mathbb{S}_{\varepsilon}$ e aponta para "dentro", pelo Teorema 4.4 .12 , segue que

$$
\operatorname{Ind}_{P H}(w, p)=\chi\left(\overline{\mathbb{B}}_{\varepsilon}(p)\right)-\chi\left(\mathbb{S}_{\varepsilon}\right) .
$$

No caso em que $m$ é par, a dimensão da esfera $\mathbb{S}_{\varepsilon}$ é $m-1$, que é ímpar, logo $\chi\left(\mathbb{S}_{\varepsilon}\right)=0$, portanto $\operatorname{Ind}_{P H}(w, p)=\chi\left(\overline{\mathbb{B}}_{\varepsilon}(p)\right)=1$.

Suponhamos agora, que $m$ é ímpar, nesse caso, a dimensão da esfera $\mathbb{S}_{\varepsilon}$ é $m-1$, que é par, $\operatorname{logo} \chi\left(\mathbb{S}_{\varepsilon}\right)=2$, portanto $\operatorname{Ind}_{P H}(w, p)=\chi\left(\overline{\mathbb{B}}_{\varepsilon}(p)\right)-\chi\left(\mathbb{S}_{\varepsilon}\right)=1-2=-1$. 


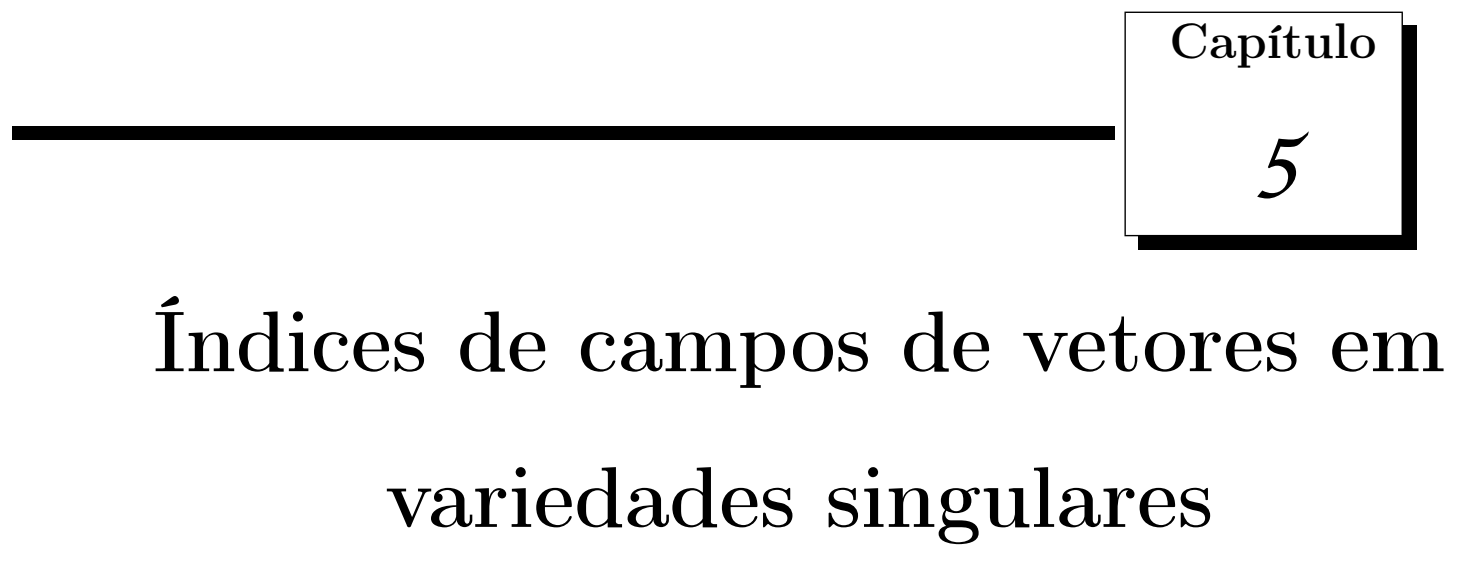

No capítulo anterior, definimos o índice de Poincaré-Hopf de $v$ em $p$, onde $v$ é um campo de vetores contínuo sobre uma variedade diferenciável $M$, e $p$ uma singularidade isolada de $v$. Além disso, mostramos que quando a variedade $M$ é compacta, e sem bordo, a soma dos índices de todas as singularidades isoladas de um campo vetorial sobre $M$ é independente do campo.

Porém, quando $V$ é uma variedade singular, o Teorema de Poincaré-Hopf não é mais verdadeiro (usando um campo de vetores qualquer). Neste caso, é necessário dar sentido a noção de campos de vetores "tangente" em uma variedade singular, e é natural que nos perguntemos, como seria a noção de "índice" de uma singularidade de um campo de vetores sobre uma variedade singular.

Neste capítulo, estudaremos alguns tipos de índices de singularidades de campos de vetores definidos em variedades singulares, tais como o índice de Schwartz e o índice GSV. Começaremos, apresentando a definição de variedade singular e exemplos mostrando que o teorema de Poincaré-Hopf não é válido em tal situação. 
Definição 5.0.14. Seja $V$ um espaço topológico, dizemos que $V$ é uma variedade singular $n$-dimensional se existe um subconjunto $S$ de $V$, tal que, $V \backslash S$ é uma variedade topológica $n$-dimensional e tal que para todo ponto $a \in S$ não existe uma vizinhança $V_{a}$ de $a$ em $V$ homeomorfa à um aberto $U$ de $\mathbb{R}^{n}$.

Definição 5.0.15. Seja $V$ uma variedade singular $n$-dimensional, chamaremos de conjunto singular de $V$ o subconjunto $S$ descrito na definição acima, e denotaremos este subconjunto por $\Sigma(V)$. Cada ponto $a \in S$ é chamado de singularidade de $V$ e diremos que uma singularidade $a$ é isolada se exister uma vizinhança $W_{a}$ de $a$ em $V$, tal que $a$ é a única singularidade de $V$ em $W_{a}$.

Exemplo 5.0.16. O toro pinçado, mergulhado em $\mathbb{R}^{3}$ admite uma singularidade no "ponto de pinçamento" $a$.

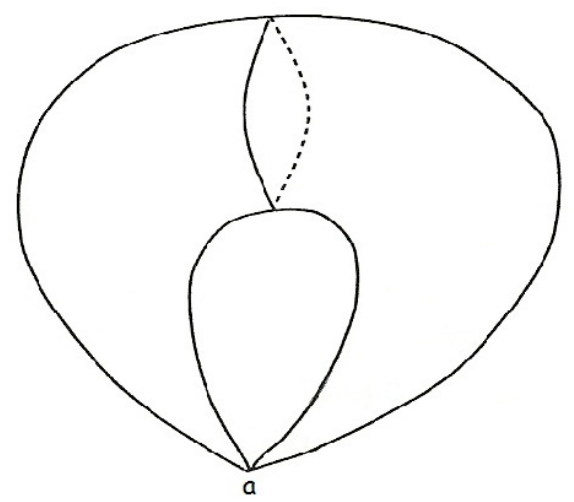

Figura 5.1: Toro pinçado com ponto de pinçamento em $a$

De fato, suponha que exista uma vizinhaça $V_{a}$ de $a$ e um homeomorfismo $h: \mathbb{B} \rightarrow V_{a}$, onde $\mathbb{B}$ é uma bola aberta de $\mathbb{R}^{2}$. Seja $b \in \mathbb{B}$ tal que $h(b)=a$, então $\tilde{h}: \mathbb{B} \backslash\{b\} \rightarrow V_{a} \backslash\{a\}$ é um homeomorfismo. Agora, como $\mathbb{B} \backslash\{b\}$ é conexa e $\tilde{h}$ é um homeomorfismo, segue que $V_{a} \backslash\{a\}$ é conexo, o que é um absurdo.

Dado um campo de vetores sobre uma variedade $V$, possivelmente com uma singularidade isolada $p$, definimos o índice de Poincaré-Hopf de $p$, apenas no caso em que $V$ era diferenciável. A seguir apresentaremos uma definição de tal índice quando $V$ é uma variedade singular.

Observação 5.0.17. Neste capítulo, trabalharemos apenas com variedades analíticas, neste caso $V \backslash \Sigma(V)$ é uma variedade diferenciável, que denotaremos por $V_{\text {reg. }}$. 
Definição 5.0.18. Seja uma variedade $V$, mergulhada em $\mathbb{R}^{n}$, com uma singularidade isolada $a$. Um campo vetorial contínuo $v$ em $V$ é uma aplicação contínua $v: V \rightarrow \mathbb{R}^{n}$ cuja imagem de cada ponto $x \in V_{\text {reg }}$ está contida no espaço linear tangente a $V_{\text {reg }}$, isto é, dado $x \in V_{\text {reg }}$, temos $v(x) \in T_{x} V_{\text {reg }}$, e $v(a)=0$.

Definição 5.0.19. Seja $V$ uma variedade singular, mergulhada em $\mathbb{R}^{n}$, com singularidade isolada em $a \in V$. Consideremos um campo de vetores contínuo $v$ sobre $V$, tal que $v$ também possa ser definido sobre uma bola fechada $\overline{\mathbb{B}}_{\varepsilon}(a)$ de centro $a$ em $\mathbb{R}^{n}$ e raio $\varepsilon$, com uma única singularidade $a$ em $\overline{\mathbb{B}}_{\varepsilon}(a)$. Definimos o índice de Poincaré-Hopf de $v$ em $a$ como sendo o índice de Poincaré-Hopf de $\tilde{v}$ em $a$, onde $\tilde{v}=\left.v\right|_{\mathbb{B}_{\varepsilon}(a)}$.

Exemplo 5.0.20. Seja $V$ o toro pinçado, mergulhado em $\mathbb{R}^{3}$, com ponto de pinçamento a. Consideremos dois exemplos de campos de vetores contínuos sobre $V$.

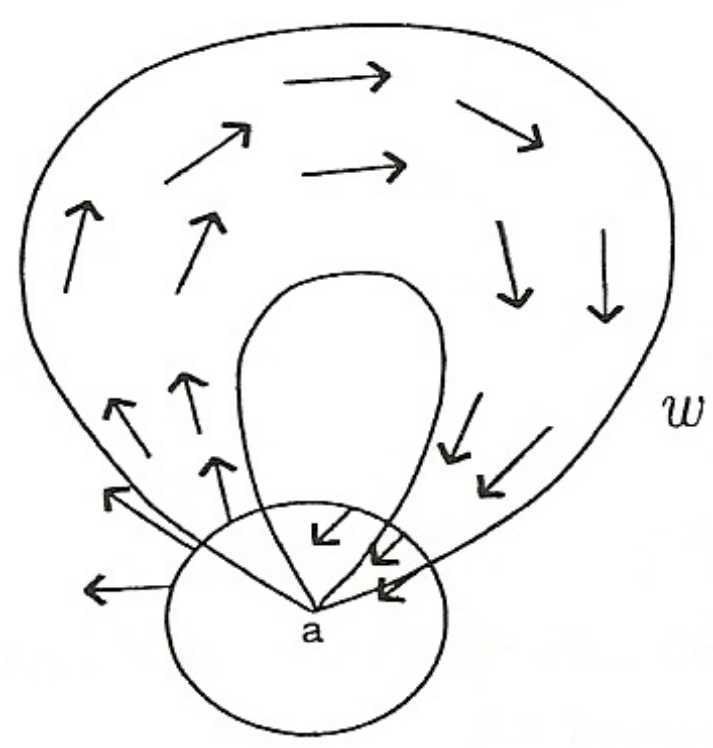

Figura 5.2: Campo $w$ sobre $V$

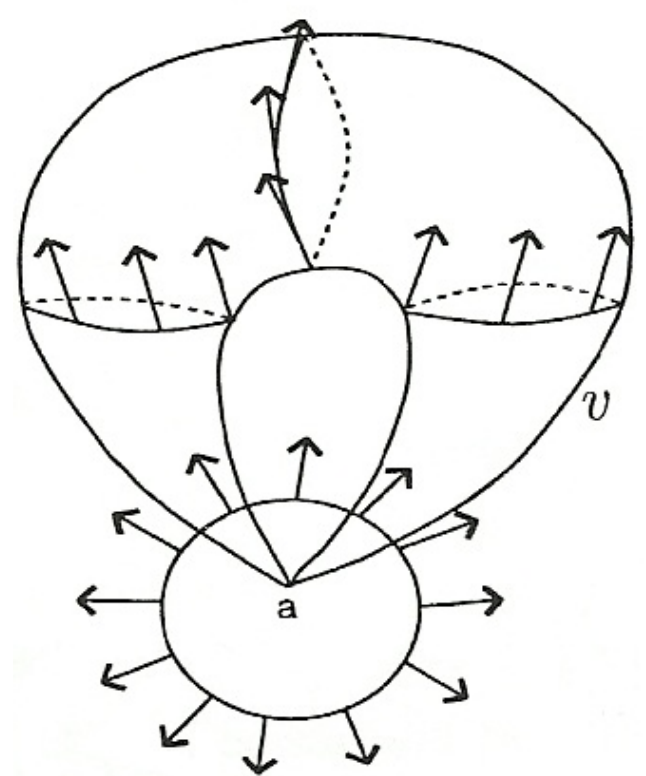

Figura 5.3: Campo $v$ sobre $V$

a) $\mathrm{O}$ campo tangente às paralelas do toro $T$ induz sobre o toro pinçado $V$ um campo $w$ entrando na bola $\overline{\mathbb{B}}_{\varepsilon}(a)$ ao longo de um dos dois círculos de interseção de $\mathbb{S}_{\varepsilon}$ com $V$ e saindo da bola ao longo do outro círculo (ver Figura 5.2. . O campo $w$ definido sobre $\mathbb{S}_{\varepsilon} \cap V$ é a restrição de um campo $\tilde{w}$ definido sobre $\mathbb{S}_{\varepsilon}$ e de índice 0 . Neste 
caso, o Teorema de Poincaré-Hopf não se verifica porque temos

$$
\chi(V)=1 \neq 0=\operatorname{Ind}_{P H}(\tilde{w}, a)
$$

b) Consideremos um campo de vetores $\tilde{v}$ sobre $\overline{\mathbb{B}}_{\varepsilon}(a)$, saindo de $\overline{\mathbb{B}}_{\varepsilon}(a)$ ao longo de $\mathbb{S}_{\varepsilon}$ e tangente a $V$ ao longo de $\overline{\mathbb{B}}_{\varepsilon}(a) \cap\left(V_{\text {reg }}\right)$. Este campo tem índice +1 em $a$, ele pode ser estendido sobre o toro pinçado em um campo de vetores $v$ sem outra singularidade além de $a$. De fato, podemos construir $v$ de tal maneira que o ângulo entre $v(x)$ e o espaço tangente ao "meridiano" contendo $x$ diminua conforme $x$ se afasta de $a$ até ser 0 (no meridiano oposto a $a$ ) como ilustra a Figura 5.3.

Neste caso, o Teorema de Poincaré-Hopf é verificado:

$$
\chi(V)=1=\operatorname{Ind}_{P H}(\tilde{v}, a) .
$$

Então, como dito acima, o Teorema de Poincaré-Hopf não é mais verdadeiro para campos de vetores quaisquer sobre uma variedade singular. Vejamos a seguir mais um exemplo em que isso acontece.

Exemplo 5.0.21. Seja a "figura oito", que denotaremos por $V$, e seja $v$ o campo de vetores sobre $V$ descrito a seguir: Observemos que $\operatorname{Ind}_{P H}(v, a)=\operatorname{Ind}_{P H}\left(v, b_{3}\right)=1$

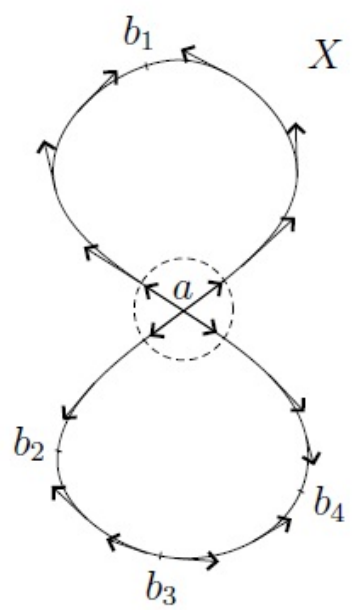

Figura 5.4: Campo sobre a figura oito. 
e que $\operatorname{Ind}_{P H}\left(v, b_{1}\right)=\operatorname{Ind}_{P H}\left(v, b_{2}\right)=\operatorname{Ind} d_{P H}\left(v, b_{4}\right)=-1, \operatorname{assim}, \sum_{i=1}^{4} \operatorname{Ind}_{P H}\left(v, b_{i}\right)+$ $\operatorname{Ind}_{P H}(v, a)=-1=\chi(V)$, ou seja nesse caso o Teorema de Poincaré-Hopf se verifica.

Observemos, que tanto no Exemplo 5.0.20 como no Exemplo 5.0.21 o caso onde o Teorema de Poincaré-Hopf continua válido é uma consequência da escolha do campo. Veremos posteriormente que classe de campos podemos utilizar para que este teorema continue válido.

A seção a seguir foi baseada no livro [2].

\subsection{O índice de Schwartz}

Índices de campos de vetores em variedades singulares foram considerados primeiramente por M.-H. Schwartz em seu estudo do Teorema de Poincaré-Hopf e de Classes de Chern para variedades singulares.

Em seu estudo, ela considerou uma classe especial de campos de vetores, que ela chamou de campos "radiais", os quais são obtidos pelo importante processo de extensão radial. Nesta seção, explicaremos a definição do correspondente índice, definido por M.H. Schwartz, no caso em que o espaço considerado é um espaço analítico equidimensional $V \subset \mathbb{C}^{m}$ com uma singularidade isolada na origem.

Definição 5.1.1. Considere um espaço analítico equidimensional $V \subset \mathbb{C}^{m}$ de dimensão $n \geq 1$ com uma singularidade isolada em 0 . Seja $\overline{\mathbb{B}}_{\varepsilon}(0)$ uma bola fechada de $\mathbb{C}^{m}$ centrada em 0 e de raio $\varepsilon$, pequena o suficiente para que toda esfera, centrada em 0 e contida em $\overline{\mathbb{B}}_{\varepsilon}(0)$, encontre $V$ transversalmente. Seja $v_{\text {rad }}$ um campo de vetores em $V \backslash\{0\}$, que seja transversal a todas as esferas $\mathbb{S}_{\varepsilon}$ centradas em 0 , para todo $\varepsilon>0$ suficientemente pequeno, e que se estenda continuamente a $\left.\mathbb{C}^{m}\right|_{V}$ com uma singularidade isolada em 0. Chamamos o campo $v_{\text {rad }}$ de campo de vetores radial em $V$.

Notemos que $v_{\text {rad }}$ pode ser estendido a um campo de vetores tangente, $v_{\text {rad }}^{\#}$, em $\overline{\mathbb{B}}_{\varepsilon}(0)$ sendo transversal a todas as esferas $\mathbb{S}_{\mathcal{\varepsilon}^{\prime}}$ centradas em 0 , onde $0<\varepsilon^{\prime} \leq \varepsilon$, obtendo assim um campo de vetores em $\overline{\mathbb{B}}_{\varepsilon}(0)$ que é radial. 
Definição 5.1.2. Por definição o indice de Schwartz de $v_{\text {rad }}$ é o índice de Poincaré-Hopf em 0 da extensão radial $v_{\text {rad }}^{\#}$, que é +1 .

Considere agora um campo de vetores contínuo $v$ em $V$ com uma singularidade isolada em 0. Queremos definir o indice de Schwartz de $v$ em 0. Este índice está relacionado "a falta de radialidade do campo", por causa disto é conhecido também como índice radial.

Podemos agora definir a diferença entre $v$ e $v_{r a d}$ em 0 : consideremos pequenas esferas $\mathbb{S}_{\varepsilon}, \mathbb{S}_{\varepsilon}^{\prime} ; \varepsilon>\varepsilon^{\prime}>0$, e seja $w$ o campo de vetores no cilindro $X$ em $V$ limitado pelos links $\mathbf{K}_{\varepsilon}=\mathbb{S}_{\varepsilon} \cap V$ e $\mathbf{K}_{\varepsilon}^{\prime}=\mathbb{S}_{\varepsilon}^{\prime} \cap V$, tal que $w$ tem um número finito de singularidades no interior de $X$, e suas restrições a $\mathbf{K}_{\varepsilon}$ e $\mathbf{K}_{\varepsilon}^{\prime}$ são $v$ e $v_{r a d}$ respectivamente. A diferença entre $v$ e $v_{r a d}$ é definida como,

$$
d\left(v, v_{r a d}\right)=\operatorname{Ind}_{P H}(w, X)
$$

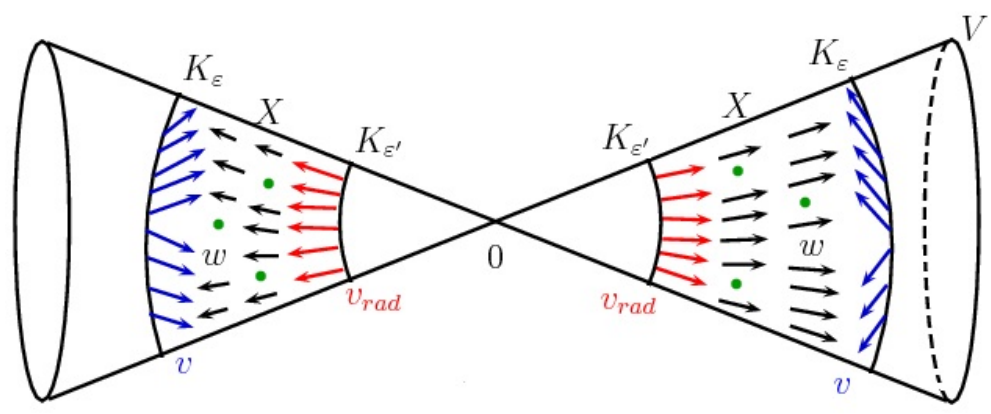

Figura 5.5: Campo de vetores w entre $K_{\varepsilon}$ e $K_{\varepsilon^{\prime}}$

Definição 5.1.3. Sejam $V \subset \mathbb{C}^{m}$ um espaço analítico equidimensional com singularidade isolada em 0 e $v$ um campo de vetores contínuo em $V$ com singularidade isolada em 0 . Definimos o índice de Schwartz de $v$ em $0 \in V$ da seguinte forma:

$$
\operatorname{Ind}_{S c h}(v, 0 ; V)=1+d\left(v, v_{r a d}\right)
$$

A seguir, apresentaremos um resultado que relaciona o índice de Schwartz com o índice de Poincaré-Hopf, e que é um "tipo" de Teorema de Poincaré-Hopf para o índice de Schwartz. 
Teorema 5.1.4. Sejam $V$ um espaço analítico equidimensional compacto, contido em $\mathbb{C}^{m}$, com singularidades isoladas $q_{1}, \ldots, q_{r}$, e seja $v$ um campo de vetores contínuo em $V$, singular nos pontos $q_{1}, \ldots, q_{r}$ e possivelmente com outras singularidades, digamos $y_{1}, \ldots, y_{s}$ de $V$. Seja,

$$
\operatorname{Ind}_{S c h}(v, V) \doteq \sum_{i=1}^{r} \operatorname{Ind}_{S c h}\left(v, q_{i}\right)+\sum_{j=1}^{s} \operatorname{Ind}_{P H}\left(v, y_{j}\right)
$$

então

$$
\operatorname{Ind}_{S c h}(v, V)=\chi(V)
$$

Demonstração: Dividiremos esta demonstração em dois casos.

Caso 1: Se o campo de vetores $v$ é radial em todas as singularidades $q_{1}, \ldots, q_{r}$, temos que

$$
\operatorname{Ind}_{S c h}\left(v, q_{i}\right)=1, \quad i=1, \ldots, r
$$

Em cada ponto singular $q_{i}$, consideremos uma bola fechada $\overline{\mathbb{B}}_{\varepsilon_{i}}\left(q_{i}\right)$ de $\mathbb{C}^{m}$, de centro $q_{i}$ e raio $\varepsilon_{i}$, suficientemente pequeno, de modo que, $q_{i}$ seja a única singularidade de $v$ em $\overline{\mathbb{B}}_{\varepsilon_{i}}\left(q_{i}\right)$.

Denotando por $\mathbb{D}_{i}$ o conjunto $V \cap \overline{\mathbb{B}}_{\varepsilon_{i}}\left(q_{i}\right)$, temos que

$$
V^{*}=V \backslash \bigcup_{i=1}^{r} \stackrel{\circ}{\mathbb{D}}_{i}
$$

é uma variedade diferenciável com bordo. Como o campo de vetores $v$ é transversal ao bordo de $V^{*}$, pelo Teorema 4.4 .12 temos

$$
\chi\left(V^{*}\right)=\sum_{j=1}^{s} \operatorname{Ind}_{P H}\left(v, y_{j}\right)
$$

Mas, sabemos que

$$
\chi(V)=\chi\left(\bigcup_{i=1}^{r} \mathbb{D}_{i}\right)+\chi\left(V^{*}\right)
$$


portanto

$$
\chi(V)=\sum_{i=1}^{r} \operatorname{Ind}_{S c h}\left(v, q_{i}\right)+\sum_{j=1}^{s} \operatorname{Ind}_{P H}\left(v, y_{j}\right) .
$$

Caso 2: Se $v$ não é radial em alguma singularidade $q_{i}$, procederemos da seguinte forma: seja $\varepsilon>0$ suficientemente pequeno, denotemos por $\overline{\mathbb{B}}_{i, \varepsilon}\left(q_{i}\right)$ a bola fechada em $\mathbb{C}^{m}$ de centro $q_{i}$ e raio $\varepsilon$, por $\mathbf{K}_{i, \varepsilon}$ o conjunto $V \cap \overline{\mathbb{B}}_{i, \varepsilon}\left(q_{i}\right)$ e por $V^{*}$ o conjunto $V \backslash \bigcup\left(V \cap \overline{\mathbb{B}}_{i, \varepsilon}\left(q_{i}\right)\right)$. Cada $\mathbf{K}_{i, \varepsilon}$ tem uma vizinhança difeomorfa a um cilindro $\mathbf{K}_{i, \varepsilon} \times[0,1]$ (ver [19]).

Tomemos $\varepsilon_{2}, \varepsilon_{1}>0$ tais que $\varepsilon>\varepsilon_{1}>\varepsilon_{2}>0$ e sejam $X_{\varepsilon, \varepsilon_{1}}, X_{\varepsilon_{1}, \varepsilon_{2}}$ os cilindros em $V$ limitados por $\left\{\mathbf{K}_{i, \varepsilon}, \mathbf{K}_{i, \varepsilon_{1}}\right\}$ e $\left\{\mathbf{K}_{i, \varepsilon_{1}}, \mathbf{K}_{i, \varepsilon_{2}}\right\}$, repectivamente. Definamos sobre o bordo destes cilindros o seguinte campo: coloquemos o campo de vetores $v$ sobre $\mathbf{K}_{i, \varepsilon_{1}}$ e em $\mathbf{K}_{i, \varepsilon}$ e $\mathbf{K}_{i, \varepsilon_{2}}$ coloquemos um campo de vetores radial $v_{r a d}$, como ilustra a Figura 5.6 .

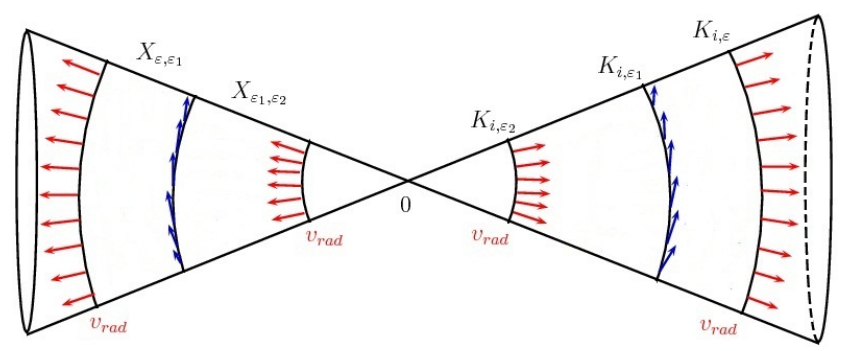

Figura 5.6: Campo de vetores em $\mathbf{K}_{i, \varepsilon_{1}}, \mathbf{K}_{i, \varepsilon}$ e $\mathbf{K}_{i, \varepsilon_{2}}$

Então, $\operatorname{Ind}_{\operatorname{Sch}}\left(v, q_{i}\right)=1+d\left(v, v_{\text {rad }}\right)$.

Denotemos por $X$ o cilindro em $V$ limitado por $\mathbf{K}_{i, \varepsilon_{2}}$ e $\mathbf{K}_{i, \varepsilon}$, que é uma variedade diferenciável com bordo. Coloquemos sobre $\mathbf{K}_{i, \varepsilon_{2}}$ e $\mathbf{K}_{i, \varepsilon}$ o campo vetorial $v$ e chamemos este campo de $w$.

Consideremos, sobre $X$, uma extensão contínua de $w$, tal que em $\mathbf{K}_{i, \varepsilon_{1}}$ esta extensão coincida com $v_{\text {rad }}$ e denotemos esta extensão ( podemos constrir uma outra extensão de $w$ sobre $X$, que não possua singularidades, logo, pelo Teorema 4.4.12, temos que $d\left(v, v_{r a d}\right)+d\left(v_{r a d}, v\right)=0$, consequentemente, este caso se reduz ao Caso 1, provando o teorema. 


\subsection{O índice GSV}

Esta seção foi baseada no capítulo 3 de [3].

Até aqui, estudamos dois tipos de índices de campos de vetores. Sendo o primeiro o índice de Poincaré-Hopf, que como vimos no Capítulo 4, é estável sobre pequenas perturbações.

Em seguida, estudamos o índice de Schwartz, definido para singularidades de campos de vetores sobre variedades singulares, e vimos, que com este índice recuperamos o Teorema de Poincaré-Hopf.

Se considerarmos agora uma variedade analítica $V$ definida por uma função holomorfa $f:\left(\mathbb{C}^{n}, 0\right) \rightarrow(\mathbb{C}, 0)$ com um ponto crítico isolado em 0 , e se $v$ é um campo de vetores em $V$, com uma singularidade isolada em 0, gostaríamos de um tipo de "índice" que, primeiramente, fosse estável sobre pequenas perturbações de ambos, a função $f$ e o campo de vetores $v$, e que satisfizesse, como o índice de Schwartz, um tipo de Teorema de Poincaré-Hopf.

Isto nos leva naturalmente para outro conceito de índice, chamado índice GSV, introduzido por X. Gómez-Mont, J. Seade e A. Verjovsky em [8, 23] para germes de hipersuperfícies e estendido em [24] (ver também [25]) para interseções completas.

Para este estudo, os autores tiveram como motivação uma questão acerca de separatrizes de campo de vetores levantada por C. Camacho. Os autores em quetão buscavam obter, a partir de um campo de vetores holomorfo sem separatriz em uma superfície singular $S$ de $\mathbb{C}^{3}$, uma extensão de tal campo, para o espaço ambiente, de forma que esta extensão fosse tangente às fibras de Milnor, e então estudar suas singularidades.

Nesta seção, definimos este índice e estudamos algumas de suas propriedades básicas. Estudamos o caso em que a variedade considerada é uma interseção completa com singularidade isolada (ICSI).

Faremos, também, um rápido estudo, sobre a maneira com que estes campos se estendem para o espaço ambiente. Assim, começaremos com uma breve discussão destes tópicos. 
Denotemos por $(V, 0)$ o germe de uma hipersuperfície analítica complexa em $\mathbb{C}^{n+1}$ dada por uma função holomorfa

$$
f:\left(\mathbb{C}^{n+1}, 0\right) \rightarrow(\mathbb{C}, 0)
$$

a qual é definida em uma pequena bola $\mathbb{B}_{\varepsilon}$, de raio $\varepsilon$, e tem um único ponto crítico em 0 .

Observação 5.2.1. Seja $f:\left(\mathbb{C}^{n+1}, 0\right) \rightarrow(\mathbb{C}, 0)$ uma função holomorfa com uma singularidade isolada em 0 e $(V, 0)$ o germe de hipersuperfície definido por $f$. O espaço tangente a um ponto $x \in V^{*}$, denotado por $T_{x} V^{*}$, é o conjunto

$$
T_{x} V^{*}=\left\{\xi \in T_{x} \mathbb{C}^{n+1}: d f_{x}(\xi)=0\right\}=\operatorname{Ker} d f_{x}
$$

Exemplo 5.2.2. Se $f$ é uma aplicação polinomial em $\mathbb{C}^{2}$ definida por $f\left(z_{1}, z_{2}\right)=z_{1}{ }^{2}+z_{2}{ }^{3}$, então a reta tangente em $V=f^{-1}(0)$ em um ponto $z=\left(z_{1}, z_{2}\right)$, que não seja a origem, é gerada pelo vetor $\xi\left(z_{1}, z_{2}\right)=\left(-3 z_{2}^{2}, 2 z_{1}\right)$.

De fato, temos que

$$
d f_{z}=2 z_{1} d z_{1}+3 z_{2}^{2} d z_{2}
$$

Logo, se

$$
d f_{z}(\xi)=2 z_{1} \xi_{1}+3 z_{2}^{2} \xi_{2}=0
$$

então,

$$
2 z_{1} \xi_{1}=-3 z_{2}^{2} \xi_{2}
$$

Como $z=\left(z_{1}, z_{2}\right) \neq 0$, suponhamos sem perca de generalidade que $z_{1} \neq 0$, logo

$$
\xi_{1}=-\frac{3 z_{2}^{2}}{2 z_{1}} \xi_{2}
$$

Tomando $\xi_{2}=2 z_{1}$, temos

$$
\left(\xi_{1}, \xi_{2}\right)=\left(-3 z_{2}^{2}, 2 z_{1}\right)
$$

Observemos que não podemos ter $z=\left(z_{1}, z_{2}\right)=\left(z_{1}, 0\right)$, pois $z \in V$. 
Agora, um campo vetorial $v$ em $V$ pode ser visto como sendo uma aplicação contínua $v:(V, 0) \rightarrow\left(\mathbb{C}^{n+1}, 0\right)$ cuja imagem está contida no espaço linear tangente a $V$ em cada ponto dado, isto é, dado $p \in V^{*}$, temos $v(p) \in T_{p} V^{*}$, em outras palavras $d f_{p}(v(p))=0$. Como $V$ é um subconjunto fechado de $B_{\varepsilon}$, pelo teorema da extensão de Tietze (ver [22]), esta aplicação se estende a uma vizinhança de $V$ em $\mathbb{C}^{n+1}$.

Geometricamente isto significa que o campo de vetores $v$ em $V$ pode sempre ser estendido ao espaço ambiente, ou equivalentemente que $v$ pode sempre ser considerado como a restrição a $V$ de um campo de vetores definido no espaço ambiente. Além disso, a extensão de $v$ em $V$ não precisa ser única. Ainda, também geram uma categoria holomorfa:

Teorema 5.2.3 ([1]). Seja $V$ uma variedade analítica complexa em $\mathbb{C}^{m}$ com uma singularidade isolada em 0. Então:

1. Existe um campo de vetores holomorfo em $V$ com uma singularidade isolada em 0 . $\mathrm{Na}$ verdade o espaço de tais campos de vetores tem dimensão infinita;

2. Se $v$ é um campo de vetores holomorfo em $V$ com uma singularidade isolada, então existem infinitas extensões de $v$ em uma vizinhança de 0 no espaço ambiente com singularidade isolada.

Como um exemplo, seja $V$ o germe de uma aplicação $f:\left(\mathbb{C}^{2}, 0\right) \rightarrow(\mathbb{C}, 0)$ que tem uma singularidade isolada na origem, então o campo de vetores $\bar{\zeta}=\left(-\frac{\partial f}{\partial z_{2}}, \frac{\partial f}{\partial z_{1}}\right)$ é tangente a $V$, pois dado $w \in V^{*}$, temos

$$
d f_{w}(\bar{\zeta}(w))=d f_{w}\left(-\frac{\partial f}{\partial z_{2}}(w), \frac{\partial f}{\partial z_{1}}(w)\right)=-\frac{\partial f}{\partial z_{1}}(w) \frac{\partial f}{\partial z_{2}}(w)+\frac{\partial f}{\partial z_{1}}(w) \frac{\partial f}{\partial z_{2}}(w)=0
$$

e seu único ponto crítico é a origem, uma vez que esta é a única singularidade de $f$.

Notemos que na verdade este campo de vetores é tangente a todas as fibras $f^{-1}(t)$, seja $\zeta$ a restrição de $\bar{\zeta}$ a $V$, note que $\zeta$ pode ser estendido para o espaço ambiente $\mathbb{C}^{2}$ de muitas maneiras; por exemplo, se $g$ é uma função holomorfa em $\mathbb{C}^{2}$ tal que $g^{-1}(0)=V$ e representa um elemento não nulo do anel local $\mathcal{O}_{2}$, então

$$
\xi=\left(g-\frac{\partial f}{\partial z_{2}}, g+\frac{\partial f}{\partial z_{1}}\right)
$$


coincide com $\zeta$ em $V$ e já não é tangente a todas as fibras de $f$, pois dado $w \in f^{-1}(t)$, com $t \neq 0$ temos

$$
d f_{w}(\xi(w))=d f_{w}\left(g(w)-\frac{\partial f}{\partial z_{2}}(w), g(w)+\frac{\partial f}{\partial z_{1}}(w)\right)=g(w) \frac{\partial f}{\partial z_{1}}(w)+g(w) \frac{\partial f}{\partial z_{2}}(w) \neq 0
$$

uma vez que $g(w) \neq 0$ e $\frac{\partial f}{\partial z_{1}}(w) \neq 0$ ou $\frac{\partial f}{\partial z_{2}}(w) \neq 0$. Além disso, escolhendo $g$ apropriadamente podemos também assegurar que $\xi$ tem uma singularidade isolada em 0 .

Em $\mathbb{C}^{3}$ temos o seguinte exemplo (ver [7]), sejam $f:\left(\mathbb{C}^{3}, 0\right) \rightarrow(\mathbb{C}, 0)$ uma função holomorfa com um ponto crítico isolado em 0 , o conjunto $V=f^{-1}(0)$ e escolha as coordenadas $\left(z_{1}, z_{2}, z_{3}\right)$ de $\mathbb{C}^{3}$ tais que $V$ intersecta o conjunto onde as derivadas parciais de $f$ com relação a $z_{2}$ e $z_{3}$ são nulas somente em 0 , isto é,

$$
V \cap\left\{\frac{\partial f}{\partial z_{2}}=\frac{\partial f}{\partial z_{3}}=0\right\}=\{0\}
$$

Defina um campo de vetores holomorfo em $\mathbb{C}^{3}$ por

$$
\bar{\zeta}=\left(f, \frac{\partial f}{\partial z_{3}},-\frac{\partial f}{\partial z_{2}}\right)
$$

note que $\bar{\zeta}$ tem uma singularidade isolada em 0 , pois

$$
V \cap\left\{\frac{\partial f}{\partial z_{2}}=\frac{\partial f}{\partial z_{3}}=0\right\}=\{0\}
$$

e

$$
d f_{w}(\bar{\zeta}(w))=d f_{w}\left(f(w), \frac{\partial f}{\partial z_{3}}(w),-\frac{\partial f}{\partial z_{2}}(w)\right)=f(w) \frac{\partial f}{\partial z_{1}}(w)
$$

consequentemente, $d f_{w}(\bar{\zeta})$ se anula nos pontos onde $f$ se anula, então $\bar{\zeta}$ é tangente a $V$.

Tomemos $\zeta=\left.\bar{\zeta}\right|_{V}$, assim temos um campo de vetores holomorfo em $V$ com uma singularidade isolada na origem, e uma extensão em $\mathbb{C}^{3}$, que também tem um singularidade isolada na origem. Contudo, ao contrário do exemplo anterior, $\bar{\zeta}$ não é mais tangente a todas as fibras de $f$. 
De fato, dado $w \in f^{-1}(t)$, com $t \neq 0$, temos

$$
d f_{w}(\bar{\zeta}(w))=f(w) \frac{\partial f}{\partial z_{1}}(w)
$$

$\operatorname{logo}, d f_{w}(\bar{\zeta}(w))=0$ se, e somente se, $\frac{\partial f}{\partial z_{1}}(w)=0$, assim $\bar{\zeta}$ seria tangente a todas as fibras de $f$ se, e somente se, $\frac{\partial f}{\partial z_{1}} \equiv 0$, mas, se $\frac{\partial f}{\partial z_{1}} \equiv 0$, então $V\left(\frac{\partial f}{\partial z_{1}}\right)=\mathbb{C}^{3}$ e consequentemente

$$
\operatorname{dim}\left(V\left(\frac{\partial f}{\partial z_{1}}, \frac{\partial f}{\partial z_{2}}, \frac{\partial f}{\partial z_{3}}\right)\right)=1 \text { ou } 2
$$

o que seria um absurdo, uma vez que $f$ tem singularidade isolada na origem.

Ainda, podemos nos esquecer que foi dado $\bar{\zeta}$ e somente considerar o campo vetorial $\zeta$ em $V$. Como $f$ se anula exatamente em $V, \zeta$ toma a forma

$$
\zeta=\left(0, \frac{\partial f}{\partial z_{3}},-\frac{\partial f}{\partial z_{2}}\right)
$$

e podemos estendê-lo a um campo de vetores holomorfo $\bar{\xi}$ em $\mathbb{C}^{3}$, definido por:

$$
\bar{\xi}=\left(0, \frac{\partial f}{\partial z_{3}},-\frac{\partial f}{\partial z_{2}}\right)
$$

Este campo é tangente a todas as hipersuperfícies não-singulares $f^{-1}(t)$. O conjunto singular de $\bar{\xi}$ é a curva $V\left(\frac{\partial f}{\partial z_{2}}, \frac{\partial f}{\partial z_{3}}\right)$, que é uma interseção completa definida pelo ideal $\left(\frac{\partial f}{\partial z_{2}}, \frac{\partial f}{\partial z_{3}}\right)$.

De fato, suponhamos que exista $g \in \mathcal{O}_{3}$ tal que $\frac{\partial f}{\partial z_{2}}=g \frac{\partial f}{\partial z_{3}}, \log \mathrm{o}$

$$
J(f)=\left\langle\frac{\partial f}{\partial z_{1}}, g \frac{\partial f}{\partial z_{3}}, \frac{\partial f}{\partial z_{3}}\right\rangle
$$

o que é um absurdo, pois $f$ tem singularidade isolada na origem e

$$
\operatorname{dim} V\left(\frac{\partial f}{\partial z_{1}}, \frac{\partial f}{\partial z_{3}}\right)=1 \text { ou } 2
$$

Agora, suponhamos que

$$
\operatorname{dim} V\left(\frac{\partial f}{\partial z_{2}}, \frac{\partial f}{\partial z_{3}}\right)=2
$$


$\log \mathrm{O}$

$$
\operatorname{dim} V\left(\frac{\partial f}{\partial z_{1}}, \frac{\partial f}{\partial z_{2}}, \frac{\partial f}{\partial z_{3}}\right)=1 \text { ou } 2
$$

o que é um absurdo, uma vez que $f$ tem singularidade isolada na origem.

Portanto, $V\left(\frac{\partial f}{\partial z_{2}}, \frac{\partial f}{\partial z_{3}}\right)$ é uma interseção completa.

Observemos que $V\left(\frac{\partial f}{\partial z_{2}}, \frac{\partial f}{\partial z_{3}}\right)$ intersecta cada fibra não-singular $f^{-1}(t)$ em um número finito de pontos, ou equivalentemente,

$$
\operatorname{dim} V\left(\frac{\partial f}{\partial z_{2}}, \frac{\partial f}{\partial z_{3}}\right) \cap f^{-1}(t)=0
$$

pois se $V\left(\frac{\partial f}{\partial z_{2}}, \frac{\partial f}{\partial z_{3}}\right) \cap f^{-1}(t)$ tivesse dimensão 1 , e se considerássemos, para cada $t \in \mathbb{C}^{*}$, a função $g:\left(\mathbb{C}^{3}, 0\right) \rightarrow(\mathbb{C}, 0)$ dada por

$$
g(x)=f(x)-t
$$

teríamos que $g^{-1}(0)=f^{-1}(t)$, e consequentemente

$$
\operatorname{dim} V\left(\frac{\partial f}{\partial z_{1}}, \frac{\partial f}{\partial z_{2}}, \frac{\partial f}{\partial z_{3}},\right) \cap f^{-1}(t)=\operatorname{dim} V\left(\frac{\partial f}{\partial z_{1}}, \frac{\partial f}{\partial z_{2}}, \frac{\partial f}{\partial z_{3}},\right) \cap g^{-1}(0)=0 \text { ou } 1
$$

o que seria um absurdo, pois todo $t \in \mathbb{C}^{*}$ é um valor regular de $f$. Além disso, a soma total (contando com as multiplicidades) dos pontos da intersecção de cada fibra não-singular $f^{-1}(t)$ com $V\left(\frac{\partial f}{\partial z_{2}}, \frac{\partial f}{\partial z_{3}}\right)$ é uma constante (como veremos mais adiante).

Esta constante é um índice que depende apenas de $\zeta$ e da maneira como $V$ é mergulhado em $\mathbb{C}^{3}$, este é o índice que estudaremos abaixo.

\section{O índice para campos de vetores sobre ICSI}

Seja $(V, 0)$ o germe de uma interseção completa $n$-dimensional reduzida com uma singularidade isolada, definida pela aplicação holomorfa

$$
f=\left(f_{1}, \ldots, f_{k}\right):\left(\mathbb{C}^{n+k}, 0\right) \rightarrow\left(\mathbb{C}^{k}, 0\right),
$$


isto é, $f_{1}, \ldots, f_{k}$ geram o ideal $I(V)$ onde $f_{i}:\left(\mathbb{C}^{n+k}, 0\right) \rightarrow\left(\mathbb{C}^{k}, 0\right), i=1, \ldots, k$. Além disso, se $n=1$ assumiremos (por enquanto) que $V$ é irredutível.

Como 0 é uma singularidade isolada de $V$, segue que os campos de vetores gradientes $\bar{\nabla} f_{1}, \ldots, \bar{\nabla} f_{k}$ são linearmente independentes fora da origem. De fato, como

$$
f=\left(f_{1}, \ldots, f_{k}\right):\left(\mathbb{C}^{n+k}, 0\right) \rightarrow\left(\mathbb{C}^{k}, 0\right)
$$

tem uma singularidade isolada na origem, e como $k+n>k$, temos que para todo $p \in \mathbb{C}^{n+k} \backslash\{0\}$

$$
d f_{p}: \mathbb{C}^{n+k} \rightarrow \mathbb{C}^{k}
$$

é uma submersão, logo a matriz jacobiana $[J f(p)]_{k \times(n+k)}$ tem $k$ linhas linearmente independes, mas as $k$ linhas de $[J f(p)]_{k \times(n+k)}$ são exatamente os vetores $\nabla f_{i}, \quad i=1, \ldots, k$.

Portanto, $\nabla f_{1}, \ldots, \nabla f_{k}$ são linearmente independentes, e consequentemente $\bar{\nabla} f_{1}, \ldots, \bar{\nabla} f_{k}$ também o são.

Temos também que $\bar{\nabla} f_{1}(0), \ldots, \bar{\nabla} f_{k}(0)$ são todos normais a $V$, pois $V=V\left(f_{1}, f_{2}, \ldots, f_{k}\right)$ e como sabemos $\nabla f_{i}(x)$ é normal a $f_{i}^{-1}(y), \quad i=1, \ldots, k, \quad \forall x \in$ $f^{-1}(y)$.

Seja $v$ um campo de vetores contínuo em $V$ com uma singularidade em 0 . O conjunto $\left\{v(x), \bar{\nabla} f_{1}(x), \ldots, \bar{\nabla} f_{k}(x)\right\}$ é um $(k+1)$-referêncial em cada ponto de $V^{*}$, e por homotopia, podemos assumir que estes vetores são ortonormais. Consequentemente, o campo de vetores $v$ define uma aplicação contínua de $V^{*}$ sobre a variedade de Stiefel $W_{k+1}(n+k)$.

Seja $\mathbf{K}=V \cap \mathbb{S}_{\varepsilon}$ o link de $V$ em 0 , temos que $\mathbf{K}$ é uma variedade suave real de dimensão $2 n-1$. De fato, consideremos $V$ e $\mathbb{S}_{\varepsilon}$ como variedades em $\mathbb{R}^{2 n}$, e a função

$$
p: \mathbb{R}^{2 n} \rightarrow \mathbb{R}
$$

dada por $p\left(x_{1}, \ldots, x_{2 n}\right)=x_{1}{ }^{2}+\ldots+x_{2 n}{ }^{2}$. Se tomarmos $r=\left.p\right|_{V^{*}}$, temos que $\varepsilon^{2}$ é um valor regular de $r$ e $r^{-1}\left(\varepsilon^{2}\right)=\mathbf{K}$. Portanto, $\mathbf{K}$ é uma variedade suave $2 n-1$ dimensional. 
Além disso, K é orientada (ver [17] pg 81), e temos uma aplicação contínua

$$
\phi_{v}=\left(v, \bar{\nabla} f_{1}, \ldots, \bar{\nabla} f_{k}\right): \mathbf{K} \rightarrow W_{k+1}(n+k) .
$$

A variedade de Stiefel $W_{k+1}(n+k)$ é difeomorfa ao espaço $U(n+k) / U(n-1)$, onde $U(n)$ denota o grupo das matrizes unitárias $n \times n$. Além disso, em [26] Steenrod mostrou que $W_{k+1}(n+k)$ é $(2 n-2)$-conexa e que $\pi_{2 n-1}\left(W_{k+1}(n+k)\right) \approx \mathbb{Z}$, assim podemos concluir que $\pi_{2 n-1}\left(W_{k+1}(n+k)\right) \approx H_{2 n-1}\left(W_{k+1}(n+k)\right)$, então a homologia de $W_{k+1}(n+k)$ na dimensão $(2 n-1)$ é isomorfa a $\mathbb{Z}$. Consideraremos, nesta seção, o caso em que $\mathbf{K}$ é uma variedade conexa, consequentemente a aplicação $\phi_{v}$ tem um grau bem definido $\operatorname{gr}\left(\phi_{v}\right) \in \mathbb{Z}$, definido por meio do homomorfismo induzido

$$
\left(\phi_{v}\right)_{*}: H_{2 n-1}(\mathbf{K}) \rightarrow H_{2 n-1}\left(W_{k+1}(n+k)\right)
$$

Definição 5.2.4. O índice GSV de $v$ em $0 \in V$, denotado por $\operatorname{Ind}_{G S V}(v, 0)$, é o grau da aplicação $\phi_{v}$.

Dada uma aplicação $f: \mathbb{C}^{n+k} \rightarrow \mathbb{C}^{k}$ podemos considerar uma fibração de Milnor associada a $f$ (ver [18, [19]), e a fibra de Milnor $\mathbf{F}$ pode ser considerada como uma variedade diferenciável compacta $2 n$-dimensional com bordo $\partial \mathbf{F}=\mathbf{K}$. Além disso, pelo Primeiro Lema de Isotopia, existe um espaço ambiente isotópico a esfera $\mathbb{S}_{\varepsilon}$ tendo $\mathbf{K}$ sobre $\partial \mathbf{F}$, o qual podemos estender para o colar de $\mathbf{K}$, que vai para um colar de $\partial \mathbf{F}$ em F. Consequentemente, $v$ pode ser considerado como um campo de vetores não-singular em $\partial \mathbf{F}$.

A seguir apresentaremos algumas propriedades do índice GSV.

Teorema 5.2.5. Sejam $(V, 0)$ o germe de uma interseção completa $n$-dimensional reduzida com uma singularidade isolada em 0 e $v$ um campo de vetores em $V$ com uma singularidade isolada em $0 \in V$, o índice GSV tem as seguintes propriedades:

(1) O índice GSV de $v$ em 0 é igual ao índice de Poincaré-Hopf de $v(\tilde{v})$ na fibra de Milnor:

$$
\operatorname{Ind}_{G S V}(v, 0)=\operatorname{Ind}_{P H}(\tilde{v}, \mathbf{F}),
$$

onde $\tilde{v}$ é a extensão de $v$ para o interior da fibra $\mathbf{F}$. 
(2) Se $v$ é transversal a $\mathbf{K}$, então

$$
\operatorname{Ind}_{G S V}(v, 0)=1+(-1)^{n} \mu
$$

onde $n$ é a dimensão complexa de $V$ e $\mu$ é o número de Milnor de 0 .

(3) Temos

$$
\mu=(-1)^{n}\left(\operatorname{Ind}_{G S V}(v, 0)-\operatorname{Ind}_{S c h}(v, 0)\right),
$$

independente da escolha de $v$.

\section{Demonstração:}

(1) Como $(V, 0)$ é uma ICSI, o campo de vetores gradiente conjungado

$$
\left\{\bar{\nabla} f_{1}, \ldots, \bar{\nabla} f_{k}\right\}
$$

são todos linearmente independentes em toda $\mathbf{F}$ e normal a esta variedade diferenciável, então o grau de $\phi_{v}$ pode ser identificado com a obstrução para estender $v$ para um campo de vetores tangente em $\mathbf{F}$;

(2) Agora, (2) segue de (1) juntamente com o Teorema 4.4.12 e do fato que, por [18, 19], a característica de Euler de $\mathbf{F}$ é $1+(-1)^{n} \mu$.

(3) Se $v$ é radial temos $\operatorname{Ind}_{S c h}(v, 0)=1$, e como de (2) temos

$$
\mu=(-1)^{n}\left(\operatorname{Ind}_{G S V}(v, 0)-1\right)
$$

segue que

$$
\mu=(-1)^{n}\left(\operatorname{Ind}_{G S V}(v, 0)-\operatorname{Ind}_{S c h}(v, 0)\right) .
$$

Seja agora, $v$ um campo de vetores qualquer. Consideremos pequenas esferas $\mathbb{S}_{\varepsilon}$, $\mathbb{S}_{\varepsilon}^{\prime} ; \varepsilon>\varepsilon^{\prime}>0$, e seja $w$ o campo de vetores no cilindro $X$ em $V$ limitado pelos links $\mathbf{K}_{\varepsilon}=\mathbb{S}_{\varepsilon} \cap V$ e $\mathbf{K}_{\varepsilon}^{\prime}=\mathbb{S}_{\varepsilon}^{\prime} \cap V$, tal que $w$ tem um número finito de singularidades $a_{i}$ no interior de $X$, e suas restrições a $\mathbf{K}_{\varepsilon}$ e $\mathbf{K}_{\varepsilon}^{\prime}$ são $v$ e $v_{\text {rad }}$ respectivamente. 


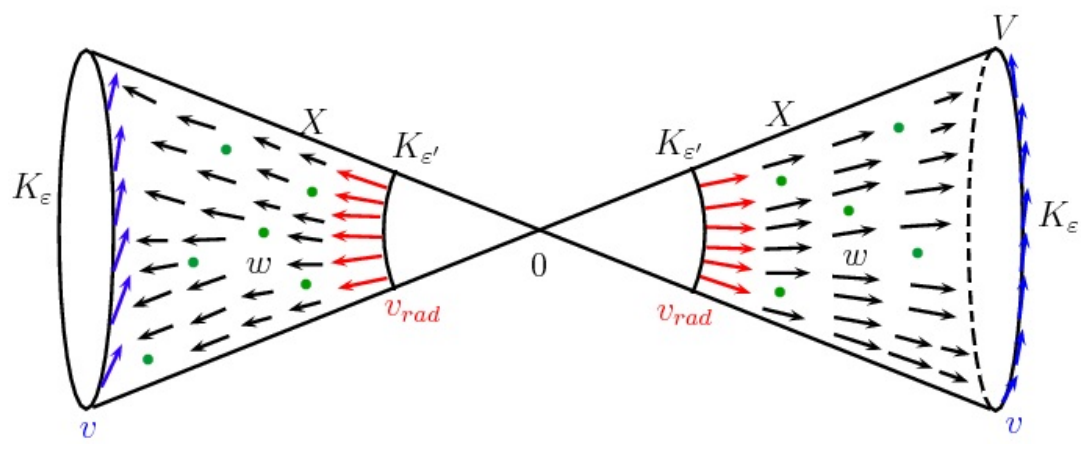

Figura 5.7: Campo de vetores w entre $K_{\varepsilon}$ e $K_{\varepsilon^{\prime}}$

Assim, temos

$$
\operatorname{Ind}_{S c h}(v, 0)=1+d\left(v, v_{r a d}\right)=\operatorname{Ind}_{S c h}\left(v_{r a d}, 0\right)+\sum_{i=1}^{r} \operatorname{Ind}_{P H}\left(w, a_{i}\right) .
$$

Agora, notemos que

$$
\operatorname{Ind}_{G S V}(v, 0)=\operatorname{Ind}_{G S V}\left(v_{r a d}, 0\right)+\sum_{i=1}^{r} \operatorname{Ind}_{P H}\left(w, a_{i}\right)
$$

De fato, como vimos antes, podemos considerar a fibra de Milnor F como uma variedade diferenciável $2 n$-dimensional com bordo $\partial \mathbf{F}=\mathbf{K}_{\varepsilon}$. Do item (1), sabemos que $\operatorname{Ind}_{G S V}(v, 0)=\operatorname{Ind}_{P H}(\tilde{v}, \mathbf{F})$, onde $\tilde{v}$ é uma extensão de $v$, que é definido em $\partial \mathbf{F}$, para o interior da fibra F. E pelo Teorema de Poincaré-Hopf, para variedades com bordo, a soma dos índices de Poincaré-Hopf $\operatorname{Ind}_{P H}(\tilde{v}, \mathbf{F})$ independe da extensão de $v$.

Então, consideremos sobre $V$ o seguinte campo $\tilde{w}$ : da origem até o $\operatorname{link} \mathbf{K}_{\varepsilon^{\prime}}$ o campo $\tilde{w}$ será igual ao campo $v_{\text {rad }}$ e no cilindro limitado pelos links $\mathbf{K}_{\varepsilon^{\prime}}$ e $\mathbf{K}_{\varepsilon}$ colocaremos $\tilde{w}=w$. Como no bordo $\partial \mathbf{F}$, o campo $\tilde{w}$ coincide com $v$, então a extensão deste campo a fibra $\mathbf{F}$ é uma extensão de $v$ a $\mathbf{F}$. Logo,

$$
\operatorname{Ind}_{G S V}(v, 0)=\operatorname{Ind}_{P H}(\tilde{w}, \mathbf{F})=\operatorname{Ind}_{G S V}\left(v_{r a d}, 0\right)+\sum_{i=1}^{r} \operatorname{Ind}_{P H}\left(w, a_{i}\right) .
$$


Assim,

$$
\begin{aligned}
\operatorname{Ind}_{G S V}(v, 0)-\operatorname{Ind}_{S c h}(v, 0) & =\operatorname{Ind}_{G S V}\left(v_{r a d}, 0\right)-\operatorname{Ind}_{S c h}\left(v_{r a d}, 0\right) \\
& =1+(-1)^{n} \mu-1=(-1)^{n} \mu
\end{aligned}
$$

e portanto,

$$
\mu=\left(\operatorname{Ind}_{G S V}(v, 0)-\operatorname{Ind}_{S c h}(v, 0)\right)(-1)^{n} .
$$

E o teorema esta demonstrado.

Observação 5.2.6. O Teorema 5.2.5 diz que se perturbarmos a aplicação $f$ adicionando uma constante pequena, então o índice é preservado, no sentido que, o indice GSV do campo de vetores sobre a fibra singular será a soma dos índices de Poincaré-Hopf em fibras vizinhas. Disto segue que a maneira como o indice GSV muda quando perturbamos $f$ não depende da escolha do campo de vetores $v$, mas somente da maneira como a topologia das fibras de Milnor muda, isto é, do comportamento do número de Milnor sobre perturbações.

A seguir, apresentaremos alguns exemplos do cálculo do índice GSV, utilizando as propriedades acima apresentadas.

Exemplo 5.2.7. No início desta seção, vimos que dada uma função

$$
f:\left(\mathbb{C}^{2}, 0\right) \rightarrow(\mathbb{C}, 0)
$$

com uma singularidade isolada na origem, o campo de vetores $v:\left(\mathbb{C}^{2}, 0\right) \rightarrow\left(\mathbb{C}^{2}, 0\right)$ dado por

$$
v(w)=\left(-\frac{\partial f}{\partial z_{2}}(w), \frac{\partial f}{\partial z_{1}} w\right)
$$

é tangente a $V=f^{-1}(0)$ e a todas as fibras de $f$, do fato de $f$ ter uma singularidade isolada na origem, juntamente com o Teorema 5.2.5, segue que $\operatorname{Ind}_{G S V}(v, 0)=0$.

Mais geralmente, consideremos uma função holomorfa

$$
f:\left(\mathbb{C}^{2 n}, 0\right) \rightarrow(\mathbb{C}, 0)
$$


com uma singularidade isolada na origem. Sua diferencial é

$$
d f_{z}=\frac{\partial f}{\partial z_{1}} d z_{1}+\ldots+\frac{\partial f}{\partial z_{2 n}} d z_{2 n}
$$

Seja $\bar{\zeta}$ um campo de vetores arbitrário obtido permutando aos pares as componentes de $d f_{z}$ e trocando o sinal de um dos componentes de cada par, por exemplo se

$$
f:\left(\mathbb{C}^{4}, 0\right) \rightarrow(\mathbb{C}, 0)
$$

podemos considerar os seguintes campos

$$
\tilde{\zeta}=\left(-\frac{\partial f}{\partial z_{2}}, \frac{\partial f}{\partial z_{1}},-\frac{\partial f}{\partial z_{4}}, \frac{\partial f}{\partial z_{3}}\right)
$$

$\mathrm{Ou}$

$$
\tilde{\zeta}=\left(-\frac{\partial f}{\partial z_{3}},-\frac{\partial f}{\partial z_{4}}, \frac{\partial f}{\partial z_{1}}, \frac{\partial f}{\partial z_{2}}\right)
$$

Em todos estes casos temos que dado $z=\left(z_{1}, \ldots, z_{2 n}\right) \in \mathbb{C}^{2 n}$

$$
d f_{z}(\tilde{\zeta}(w))=0
$$

para todo $w \in \mathbb{C}^{2 n}$, isto significa que todos estes campos de vetores são tangentes a todas as fibras de $f$, em particular a $V=f^{-1}(0)$, assim seu índice GSV em 0 é zero.

Exemplo 5.2.8 ([7]). Denote as coordenadas de $\mathbb{C}^{2 n+1}$ por $\left(z_{0}, z_{1}, \ldots, z_{2 n}\right)$, dada uma função holomorfa

$$
f:\left(\mathbb{C}^{2 n+1}, 0\right) \rightarrow(\mathbb{C}, 0)
$$

com uma singularidade isolada em 0 , considere o campo de vetores:

$$
\tilde{\zeta}=\left(f, \frac{\partial f}{\partial z_{2}},-\frac{\partial f}{\partial z_{1}}, \ldots, \frac{\partial f}{\partial z_{2 n}},-\frac{\partial f}{\partial z_{2 n-1}}\right) .
$$


Vamos assumir que as coordenadas escolhidas são tais que $\tilde{\zeta}$ tenha uma singularidade isolada na origem, isto é,

$$
V \cap\left(\bigcap_{i=1}^{2 n}\left\{\frac{\partial f}{\partial z_{i}}=0\right\}\right)=\{0\}
$$

$\operatorname{Seja} \zeta=\left.\tilde{\zeta}\right|_{V}$, note que

$$
d f(\tilde{\zeta})=f \frac{\partial f}{\partial z_{0}}
$$

consequentemente $\zeta$ é tangente a $V$ e é também a restrição a $V$ do campo de vetores

$$
\tilde{\xi}=\left(0, \frac{\partial f}{\partial z_{2}},-\frac{\partial f}{\partial z_{1}}, \ldots, \frac{\partial f}{\partial z_{2 n}},-\frac{\partial f}{\partial z_{2 n-1}}\right)
$$

definido no espaço ambiente e o qual é tangente a todas as hipersuperfícies não-singulares $f^{-1}(t)$.

O conjunto singular de $\tilde{\xi}$ é a interseção completa definida pelo ideal

$$
\left(\frac{\partial f}{\partial z_{1}}, \ldots, \frac{\partial f}{\partial z_{2 n}}\right)
$$

o qual encontra cada fibra não-singular $f^{-1}(t)$ em uma quantidade finita de pontos. Do Teorema 5.2.5, segue que a soma total destes pontos (contando com suas multiplicidades) é o índice GSV de $\zeta$ em $V$.

Observação 5.2.9. É possível mostrar que este índice pode ser calculado pela fórmula:

$$
\operatorname{Ind}_{G S V}(\zeta, 0 ; V)=\operatorname{dim}_{\mathbb{C}} \frac{\mathcal{O}_{2 n+1}}{\left(f, \frac{\partial f}{\partial z_{1}}, \ldots, \frac{\partial f}{\partial z_{2 n}}\right)},
$$

isto é, $\operatorname{Ind}_{G S V}(\zeta, 0 ; V)$ é a dimensão do espaço vetorial complexo de germes de funções holomorfas em 0 dividido pelo ideal

$$
\left(f, \frac{\partial f}{\partial z_{1}}, \ldots, \frac{\partial f}{\partial z_{2 n}}\right)
$$


Exemplo 5.2.10. Considere agora um polinômio Pham-Brieskorn em $\mathbb{C}^{n+1}$ com coordenadas $\left(z_{1}, \ldots, z_{n+1}\right)$, que é um polinômio

$$
f(z)=z_{1}^{d_{1}}+\ldots+z_{n+1}^{d_{n+1}}
$$

onde $d_{i} \geq 2$ e $d_{i} \in \mathbb{Z}$, para todo $i=1, \ldots, n+1$. A variedade

$$
V=f^{-1}(0)=\left\{z=\left(z_{1}, \ldots, z_{n+1}\right) \in \mathbb{C}^{n+1} ; z_{1}^{d_{1}}+\ldots+z_{n+1}{ }^{d_{n+1}}=0\right\}
$$

é uma hipersuperfície de dimensão complexa $n$ com uma singularidade isolada em $0 \in$ $\mathbb{C}^{n+1}, \operatorname{logo} V$ é uma $I C S I$.

Sejam $d$ o mínimo múltiplo comum $\operatorname{dos} d_{i}, q_{i}=d / d_{i}, i=1, \ldots, n+1$, e considere a ação do grupo multiplicativo $\mathbb{C}^{*}$ sobre $\mathbb{C}^{n+1}$ dada por:

$$
t \cdot\left(z_{1}, \ldots, z_{n+1}\right)=\left(t^{q_{1}} z_{1}, \ldots, t^{q_{n+1}} z_{n+1}\right)
$$

Para cada $t \in \mathbb{C}^{*}$, temos

$$
f\left(t \cdot\left(z_{1}, \ldots, z_{n+1}\right)\right)=f\left(t^{q_{1}} z_{1}, \ldots, t^{q_{n+1}} z_{n+1}\right)=t^{d} \cdot f\left(z_{1}, \ldots, z_{n+1}\right) .
$$

Lembrando que, dado $\left(z_{1}, \ldots, z_{n+1}\right) \in \mathbb{C}^{n+1}$ sua órbita é o conjunto

$$
\left\{t \cdot\left(z_{1}, \ldots, z_{n+1}\right) ; \quad t \in \mathbb{C}^{*}\right\}
$$

temos por $(5.2 .1)$ que se $\left(z_{1}, \ldots, z_{n+1}\right) \in V$, então $\left\{t \cdot\left(z_{1}, \ldots, z_{n+1}\right)\right\} \subset V$. Consequentemente, $V$ é um conjunto invariante por esta ação, isto é, $V$ é união de $\mathbb{C}^{*}$-órbitas.

Todo fluxo holomorfo determina um campo de vetores holomorfo, além disso, podemos mostrar que o campo de vetores em $V$ correspondente a $\mathbb{C}^{*}$-ação acima, que denotaremos por $v_{r a d}$, é radial em $V \backslash\{0\}$. Assim, se todos os $d_{i}^{\prime} s$ são iguais, de forma que $V$ é homogênio, então o campo de vetores $v_{\text {rad }}$ é o campo de vetores radial usual $\left(z_{1}, \ldots, z_{n+1}\right)$ 
a menos de uma constante. Então, pelo Teorema 5.2.5 temos:

$$
\operatorname{Ind}_{G S V}\left(v_{\text {rad }}, 0 ; V\right)=1+(-1)^{n} \mu(V),
$$

$\mu(V)$ é o número de Milnor de $V$. De [19], sabemos

$$
\mu(V)=\left(d_{1}-1\right)\left(d_{2}-1\right) \ldots\left(d_{n+1}-1\right)
$$

Consequentemente,

$$
\operatorname{Ind}_{G S V}\left(v_{r a d}, 0 ; V\right)=1+(-1)^{n}\left(d_{1}-1\right)\left(d_{2}-1\right) \ldots\left(d_{n+1}-1\right) .
$$

Então, concluímos que:

(i) Se $n$ é par, $\operatorname{Ind}_{G S V}\left(v_{r a d}, 0 ; V\right)>0$, então toda extensão de $v_{r a d}$ à um campo de vetores contínuo no espaço ambiente, tangente a alguma fibra de $f$ (ou a todas elas) terá singularidades em cada fibra onde ele é tangente;

(ii) Se $n$ é ímpar e o menor dos $d_{i}{ }^{\prime} s$ é $\geq 3$, então $\operatorname{Ind}_{G S V}\left(v_{r a d}, 0 ; V\right)<0$. Isto significa que $v_{\text {rad }}$ não pode ser extendido a um campo de vetores holomorfo no espaço ambiente sendo tangente a uma fibra de $f$ e tendo singularidade isolada nesta fibra. Se pudessemos fazer isso, então o índice seria positivo;

(iii) Se $n$ é ímpar e todos os $d_{i}{ }^{\prime} s$ são 2 , então $\mu(V)=1$, e consequentemente

$$
\operatorname{Ind}_{G S V}\left(v_{r a d}, 0 ; V\right)=0
$$

e podemos extender $v_{r a d}$ para o espaço ambiente sendo tangente e não-singular nas fibras de $f$.

Apresentaremos agora, outra possível generalização do Teorema de Poincaré-Hopf para o caso singular. 
Teorema 5.2.11. Sejam $V$ uma variedade analítica complexa compacta com singularidades isoladas $x_{1}, \ldots, x_{r}$, as quais são todas germes de interseção completa com singularidade isolada (reduzida), seja $v$ um campo de vetores contínuo em $V$, singular nos pontos $x_{1}, \ldots, x_{r}$ e possivelmente em alguns outros pontos regulares, digamos $y_{1}, \ldots, y_{s}$ de $V$. Seja $\operatorname{Ind}_{G S V}(v, V)$ o índice GSV total de $v$, isto é

$$
\operatorname{Ind}_{G S V}(v, V)=\sum_{i=1}^{r} \operatorname{Ind}_{G S V}\left(v, x_{i}\right)+\sum_{j=1}^{s} \operatorname{Ind}_{P H}\left(v, y_{j}\right)
$$

Então, temos:

$$
\operatorname{Ind}_{G S V}(v, V)=\chi(V)+(-1)^{n} \sum_{i=1}^{r} \mu\left(x_{i}\right),
$$

onde $\mu\left(x_{i}\right)$ é o número de Milnor de $V$ em $x_{i}$.

Demonstração: Pelo Teorema 5.2.5 sabemos que

$$
(-1)^{n} \mu\left(x_{i}\right)+\operatorname{Ind}_{S c h}\left(v, x_{i}\right)=\operatorname{Ind}_{G S V}\left(v, x_{i}\right),
$$

então

$$
\operatorname{Ind}_{G S V}(v, V)=\sum_{i=1}^{r} \operatorname{Ind}_{S c h}\left(v, x_{i}\right)+\sum_{j=1}^{s} \operatorname{Ind}_{P H}\left(v, y_{j}\right)+(-1)^{n} \sum_{i=1}^{r} \mu\left(x_{i}\right) .
$$

Do Teorema 5.1.4, segue que

$$
\operatorname{Ind}_{G S V}(v, V)=\chi(V)+(-1)^{n} \sum_{i=1}^{r} \mu\left(x_{i}\right),
$$

como queríamos mostrar. 


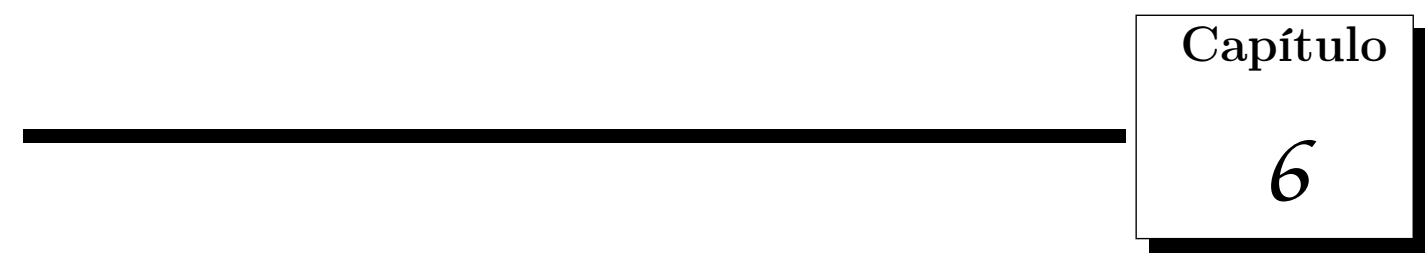

\section{Conclusão}

Neste trabalho, inicialmente estudamos o índice de Poincaré-Hopf de singularidades isoladas de campos de vetores definidos em variedades diferenciáveis. O principal resultado na primeira etapa, foi o supreendente Teorema de Poincaré-Hopf, que faz uma conexão entre ramos distintos da matemática.

No caso de campos de vetores definidos em variedades singulares, investigamos a noção de índice de Schwartz, que nos permitiu recuperar o Teorema de Poincaré-Hopf.

No caso específico de campos de vetores definidos em uma interseção completa com singularidade isolada, estudamos também o índice GSV, e como vimos, este índice está relacionado com o índice de Poincaré-Hopf, com o índice de Schwartz e com o número de Milnor, que é um invariante central em teoria de singularidades. Além disso, assim como o índice de Schwartz, o índice GSV nos permite recuperar uma versão do Teorema de Poincaré-Hopf.

Em várias áreas da matemática existem diversas situações em que o estudo de campos de vetores é crucial para o desenvolvimento científico. Este trabalho fornece as ferramentas básicas para o estudo de campos de vetores definidos tanto em variedades diferenciáveis como singulares. 



\section{Referências Bibliográficas}

[1] Ch. Bonatti e X. Gómez-Mont, The index of a holomorphic vector field on a singular variety, Astérisque 222, 1994, 9-35.

[2] J.-P. Brasselet, Characteristic classes and singular varieties, livro a aparecer.

[3] J.-P. Brasselet, J. Seade e T. Suwa, Vector Fields on singular varieties, Lecture Notes in Mathematics. Springer-Verlag, Berlin, 2009.

[4] K. Burns e M. Gidea, Differential geometry and topology with a view to dynamical systems, Chapman e Hall/CRC, 2005.

[5] M. P. do Carmo, Formas diferenciáveis e aplicações, $8^{\circ}$ Colóquio brasileiro de Matemática, Poços de Caldas, 1971.

[6] C.G. Gibson, K. Wirthmuller, A.A du Plessis and E.J.N. Looijenga, Topological stability of smooth mappings, Lecture Notes in Mathematics, 552.

[7] X. Gómes-Mont, An algebraic formula for the index of a vector field on a hypersurface with an isolated singularity, J. Algebraic Geom. 7, 1998, 731-752.

[8] X. Gómes-Mont, J. Seade e A. Verjovsky, The index of a holomorphic flow with an isolated singularity, Math. Ann. 291, 1991, 737-751.

[9] P. Griffiths, J. Harris Principles of algebraic geometry, John Wiley e sons, New York, 1978. 
[10] R. Gunning, H. Rossi, Analytic functions of several complex variables. New Jersey: Prentice-Hall, 1965.

[11] H. Hamm, Lokale topologische Eigenschaften komplexer Räume, Math. Ann. 191, 1971, 235-252.

[12] R. Hartshorne, Algebraic geometry, Graduate Texts in Mathematics 52, Springer Verlag - New York Heidelberg Berlin.

[13] M. W. Hirsch, Differential topology, Springer - Verlag - New York Heidelberg Berlin, 1976.

[14] E. D. Judice, O Teorema de Sard e suas aplicações, Tese de Concurso - Belo Horizonte, 1961.

[15] E. L. Lima, Curso de análise, vol.2, Projeto Euclides, IMPA, 2008.

[16] E. L. Lima, Introdução à topologia diferencial, Notas de Matemática no 23, IMPA, 1961.

[17] E. L. Lima, Variedades diferenciáveis, Publicações matemáticas, IMPA, 2007.

[18] E.J.N. Looijenga, Isolated singular points on complete intersections, London Mathematical Society - Lectures Notes Series 77.

[19] J. Milnor, Singular points of complex hypersurfaces, Princeton University Press, 1968.

[20] J. Milnor e J. Stasheff, Characteristic classes, Princeton University Press, 1974.

[21] J.R. Munkres, Elements of algebraic topology, Perseus Publishing, 1984.

[22] J.R. Munkres, Topology, Second Edition, Prentice Hall, 2000.

[23] J. Seade, The index if a vector field on a complex surface with singularities, in "The Lefschetz Centennial Conf.", ed. A. verjovsky, Contemp. Math. 58, Part III, Amer. Math. Soc. 1987, 225-232. 
[24] J. Seade e T. Suwa, A residue formula for the index of a holomorphic flow, Math. Annalen 304, 1996, 621-634.

[25] J. Seade e T. Suwa, An adjunction formula for local complete intersections, Internat. J. Math. 9, 1998, 759-768.

[26] N. Steenrod, The topology of fibre bundles, Princeton University Press, 1951.

[27] F. Tari, Singularidades de aplicações diferenciáveis. São Carlos: Notas didáticas 34 ICMC-USP, 1999.

[28] J. Whitehead, On $\mathbb{C}^{1}$ complexes, Annals of Math. 41, 1940, 809-824. 